\title{
NOV 51964
}

minn from the litrary.

\section{NBS}

Eechnical Note

BIBLIOGRAPHY ON THE MEASUREMENT OF BULK RESISTIVITY OF SEMICONDUCTOR MATERIALS

FOR ELECTRON DEVICES

JUDSON C. FRENCH

U. S. DEPARTMENT OF COMMERCE

NATIONAL BUREAU OF STANDARDS 


\section{THE NATIONAL BUREAU OF STANDARDS}

The National Bureau of Standards is a principal focal point in the Federal Government for assuring maximum application of the physical and engineering sciences to the advancement of technology in industry and commerce. Its responsibilities include development and maintenance of the national standards of measurement, and the provisions of means for making measurements consistent with those standards; determination of physical constants and properties of materials; development of methods for testing materials, mechanisms, and structures, and making such tests as may be necessary, particularly for government agencies; cooperation in the establishment of standard practices for incorporation in codes and specifications; advisory service to government agencies on scientific and technical problems; invention and development of devices to serve special needs of the Government; assistance to industry, business, and consumers in the development and acceptance of commercial standards and simplified trade practice recommendations; administration of programs in cooperation with United States business groups and standards organizations for the development of international standards of practice; and maintenance of a clearinghouse for the collection and dissemination of scientific, technical, and engineering information. The scope of the Bureau's activities is suggested in the following listing of its four Institutes and their organizational units.

Institute for Basic Standard8. Electricity. Metrology. Heat. Radiation Physics. Mechanics. Applied Mathematics. Atomic Physics. Physical Chemistry. Laboratory Astrophysics. ${ }^{*}$ Radio Standards Laboratory: Radio Standards Physics; Radio Standards Engineering. ${ }^{*}$ Office of Standard Reference Data.

Institute for Materials Research. Analytical Chemistry. Polymers. Metallurgy. Inorganic Materials. Reactor Radiations. Cryogenics.** Office of Standard Reference Materials.

Central Radio Propagation Laboratory.** Ionosphere Research and Propagation. Troposphere and Space Telecommunications. Radio Systems. Upper Atmosphere and Space Physics.

Institute for Applied Technology. Textiles and Apparel Technology Center. Building Research. Industrial Equipment. Information Technology. Performance Test Development. Instrumentation. Transport Systems. Office of Technical Services. Office of Weights and Measures. Office of Engineering Standards. Office of Industrial Services.

* NBS Group, Joint Institute for Laboratory Astrophysics at the University of Colorado.

** Located at Boulder, Colorado. 


\title{
NATIONAL BUREAU OF STANDARDS Technical Note 232
}

ISSUED OCTOBER 21, 1964

\section{BIBLIOGRAPHY ON THE MEASUREMENT OF BULK RESISTIVITY OF SEMICONDUCTOR MATERIALS FOR ELECTRON DEVICES}

\author{
Judson C. French
}

NBS Technical Notes are designed to supplement the Bureau's regular publications program. They provide a means for making available scientific data that are of transient or limited interest. Technical Notes may be listed or referred to in the open literature. 
Contents

\section{Page}

1. Introduction. . . . . . . . . . . . . . . . . 1

2. Index to Subject Matter . . . . . . . . . . . . 2 2a. Index Categories . . . . . . . . . . . . . 3 2b. Index Tabulation ................ 4

3. Index to Authors. . . . . . . . . . . . . . . 7

4. Bibliography. . . . . . . . . . . . . . . . 13 
Bibliography on the lleasurement of isulk Resistivity of Semiconductor Haterials for Electron Devices

Judson C. French

In support of a study of accurate measurements of the bulk resistivity of semiconductor materials such as germanium and silicon which are used in electron devices, a literature search has led to the development of a rather large collection of references. The file cards making up the bibliography are reproduced in this Teclmical iote for the assistance of others who are concerned with the improvement and standardization of these measurements.

\section{Introduction}

In the course of a study of accurate measurements of the bulk resistivity of semiconductor materials for use in electron devices, for example, germanium and silicon, a search of the literature has been conducted. I The result has been the accumulation of quite a number of references which appear to offer promise of being helpful to the study. The subject matter includes topics which are obviously pertinent, such as neasurement procedures and correction factors, as well as topics of a more indirectly applicable nature, such as sample preparation techniques and dimensional measurements, surface and pressure effects, uniformity, and so on. In regard to the former category, the collection is believed to be quite complete through 1963; a number of entries in the period to about mid-1964 are also included. The entries in the latter category are only those which have been selected as being of present or anticipated application to some of the specific problems of this study.

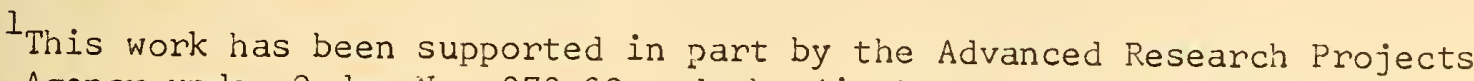
Agency under Order No. 373-62 and the Air Force Cambridge Research Laboratories under PRO 61-560 and 62-200. 
Nevertheless, others who are concerned with semiconductor resistivity measurements have expressed interest in having the collection made available for their use. For this reason, the file cards which form the working bibliography have been reproduced in this Tecinical Note with the hope that they will indeed be helpful in this field.

Because of the overlapping nature of the topics in many of the articles, no attempt has been made to group the cards by subject matter. Instead, they are arranged alphabetically on the basis of the author's name. An abstract is included as a guide to the article's content. This is frequently the author's abstract. To provide a brief index to the material covered by the references, a breakdown of the bibliography into thirteen categories of especial interest to the present study has been made. This has been based in many instances only on the statement in the abstract. It is recommended that this index be considered only to be a means of quickly finding the major references in these categories. It provides a starting point in seeking a complete set of references on a given topic. The identification numbers of the cards, which are used in the index, are to be found typed near the upper right hand corners of the cards.

Although the cards nave been reproduced on both sides of a page, they may be cut out and attached to suitable file cards using two copies of the Technical itote if this should be desirable to provide a working file.

2. Index to Subject Matter

The subject matter of the collected references has been divided into the thirteen categories listed on page 3 in Section 2a. In Section $2 \mathrm{~b}$ on pages 4 to 6 , the identification numbers of appropriate cards are listed below the numbers corresponding to these categories. 


\section{2a. Index Categories}

1. Bulk resistivity measurement: dc methods requiring probes or other contacts.

2. Bulk resistivity measurement: ac methods requiring probes or other contacts. (See also Category 1 for applicable techniques.)

3. Bulk resistivity measurement: ac contactless methods, excluding microwave frequencies, but including measurements of related properties such as dielectric constant and carrier lifetimes.

4. Bulk resistivity measurement: microwave methods, including microwave measurements of related properties such as dielectric constant and carrier lifetimes.

5. Bulk resistivity measurement: other methods than in Categories 1 to 4 .

6. Layer resistivity measurement, including epitaxial and diffused layers. (See also Categories 1 to 5 for bulk measurement methods applicable to thin samples.)

7. Resistivity measurements at resistivities of $100 \mathrm{ohm}-\mathrm{cm}$ or greater.

3. Material properties, excluding inhomogeneities and anisotropies, but including surface and contact phenomena (see also Category 10), probe wear effects, mobility and lifetime measurements not included elsewhere, the dependence of resistivity on pressure, temperature and nuclear radiation, etc.

9. Sample inhomogeneities and anisotropies, including growth methods and defect determination.

10. Sample preparation and associated techniques, including crystal orientation, sawing, lapping, polishing, dimensional measurement, plating, application of leads, surface evaluation, etc. (See also Category 8 , especially for contact phenomena.)

11. Miscellaneous equipment and techniques.

12. Correction factors, mathematical principles, and error analysis. (See also references on the specific method of measurement of interest. This category generally excludes papers with a theoretical introduction to a described method of measurement unless extensive correction factors are tabulated also.)

13. Survey articles. 
2b. Index Tabulation

Categories $1-6$

\begin{tabular}{|c|c|c|c|c|c|c|c|c|}
\hline \multicolumn{3}{|c|}{1} & 2 & 3 & \multicolumn{2}{|c|}{4} & \multirow{2}{*}{$\frac{5}{\mathrm{~A} 3}$} & \multirow{2}{*}{$\frac{6}{A 6}$} \\
\hline A4 & L7 & V3 & A6 & B25 & A 8 & K27 & & \\
\hline A5 & Ll3 & V4 & A7 & C2 & A9 & LI & A6 & Al2 \\
\hline A7 & M2 & V5 & BII & C10 & Alo & L8 & B9 & BI \\
\hline A9 & M6 & V6 & C6 & D9 & All & L10 & B14 & B18 \\
\hline AlO & M9 & V8 & D9 & D14 & Al5 & Lll & В30 & B30 \\
\hline All & MII & V10 & D16 & D16 & B5 & L12 & D14 & B34 \\
\hline $\mathrm{Al} 2$ & N7 & WI & F9 & G7 & B9 & M21 & D16 & $\mathrm{Cl3}$ \\
\hline $\mathrm{Al3}$ & 03 & W3 & L13 & G13 & $\mathrm{B} 10$ & $\mathrm{NI}$ & E2 & D7 \\
\hline B2 & 05 & W7 & P3 & HII2 & $\mathrm{B} 13$ & N2 & F3 & D16 \\
\hline B6 & P3 & W9 & P16 & I3 & B17 & N3 & F6 & Fll \\
\hline B15 & P6 & W13 & P17 & K28 & B28 & N4 & G3 & Gl \\
\hline B22 & $\mathrm{P} 12$ & W14 & R19 & K29 & B29 & N5 & G7 & G6 \\
\hline B26 & P13 & W16 & R20 & M6 & C5 & $\mathrm{N} I 1$ & Gll & GlI \\
\hline В30 & P16 & & V12 & $M 20$ & C7 & RI & H3 & H9 \\
\hline $\mathrm{C} 4$ & R7 & & & iN 10 & C8 & R2 & K8 & 1123 \\
\hline $\mathrm{Cll}$ & R9 & & & N13 & C9 & R4 & K9 & I4 \\
\hline $\mathrm{Cl6}$ & RI2 & & & 01 & $\mathrm{ClO}$ & R16 & K15 & KI \\
\hline $\mathrm{C} 20$ & $\mathrm{R} 18$ & & & 04 & $\mathrm{Cl3}$ & $\mathrm{S} 2$ & $\mathrm{~K} 26$ & $\mathrm{~K} 7$ \\
\hline D6 & R19 & & & P15 & D9 & $\mathrm{S} 21$ & L14 & K19 \\
\hline D7 & R20 & & & P16 & D16 & $\mathrm{S} 29$ & M12 & $\mathrm{K} 23$ \\
\hline Dll & S9 & & & P17 & $\mathrm{Fl}$ & S30 & 01 & K25 \\
\hline D13 & S10 & & & P19 & Fll & S3.9 & 06 & $\mathrm{~K} 26$ \\
\hline D14 & SII & & & R19 & F15 & $\mathrm{Tl}$ & 07 & K28 \\
\hline D16 & $\mathrm{S} 20$ & & & S26 & G13 & T2 & 08 & L3 \\
\hline E7 & S22 & & & TI & Gl7 & T24 & P2 & M6 \\
\hline F2 & S24 & & & T24. & G18 & $\mathrm{Z3}$ & P8 & MI2 \\
\hline F6 & S27 & & & N6 & HIl & & R5 & iN5 \\
\hline F8 & $\mathrm{S} 28$ & & & Y1 & $\mathrm{H} 20$ & & R6 & N7 \\
\hline G3 & S33 & & & $\mathrm{Z} 2$ & 1121 & & R20 & $\mathrm{Pl}$ \\
\hline $\mathrm{Gll}$ & S35 & & & & $\mathrm{H} 22$ & & S22 & P15 \\
\hline $\mathrm{H} 4$ & 540 & & & & $\mathrm{H} 25$ & & S40 & P21 \\
\hline H5 & S42 & & & & $\mathrm{H} 27$ & & 541 & R6 \\
\hline H6 & S46 & & & & $\mathrm{H} 28$ & & S50 & R18 \\
\hline H7 & 547 & & & & I3 & & T 8 & S9 \\
\hline $\mathrm{H} 15$ & 548 & & & & $\mathrm{Jl}$ & & W16 & S10 \\
\hline $\mathrm{H} 19$ & 549 & & & & J2 & & & Sll \\
\hline $\mathrm{H} 23$ & S50 & & & & J3 & & & S16 \\
\hline H126 & T5 & & & & $J_{4}$ & & & S33 \\
\hline I3 & T6 & & & & J5 & & & S35 \\
\hline K5 & T19 & & & & K2 & & & S42 \\
\hline K6 & T20 & & & & $\mathrm{K} 17$ & & & S47 \\
\hline K7 & Ul & & & & K18 & & & S49 \\
\hline K14 & $\mathrm{Vl}$ & & & & K19 & & & T8 \\
\hline K25 & V2 & & & & K24 & & & T18 \\
\hline & & & & & & & & T19 \\
\hline
\end{tabular}


2b. Index Tabulation

Categories $7-9$

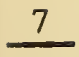

A7

B22

C5

F9

HI9

I3

J3

J5

K8

K29

$\mathrm{N} 10$

P12

RI6

R19

T6
8

\begin{tabular}{llll}
\hline A1 & F12 & N8 & V9 \\
A2 & F13 & N10 & V11 \\
A14 & F14 & O2 & V12 \\
A16 & F16 & P1 & W2 \\
B2 & G2 & P4 & W5 \\
B5 & G4 & P5 & W7 \\
B7 & G7 & P7 & W8 \\
B8 & G9 & P10 & W10 \\
B10 & G13 & P14 & W15 \\
B15 & G15 & P16 & W17 \\
B19 & G16 & P18 & Y1 \\
B20 & H2 & P20 & Z1 \\
B21 & H6 & P21 & Z3
\end{tabular}

B27 I2 R9

$\begin{array}{lll}\text { B29 } & \text { I4 } & \text { RI0 } \\ \text { B33 } & \text { I5 } & \text { RII }\end{array}$

C2 J6

C6 J7

C9 J8

C12 J9

C14

C15

C17

$\mathrm{Cl} 8$

$\mathrm{C} 20$

C22

DI

D2

D4

D5

D9

D15

D16

D17

D18

D19

El

E5

E6

E7

F4

F5

F9

$\mathrm{KI}$

K4

$\mathrm{KII}$

$\mathrm{K} 13$

K16

K22

K24

L5

L14

L15

L16

M3

M4

M5

147

M13

M17

M19

M21

M22

M23

NI

N6
RI2

RI3

RI4

RI5

RI8

R2I

SI

S5

S6

57

S8

S18

S2 4

S25

S28

S29

S35

S40

S4I

$\mathrm{Tl}$

T2

T3

T4

T7

T10

TII

TI 4

V7
9

Al

H8

S19

A4

A5

$\mathrm{HIO}$

S23

Al2

HI3

S24

A 15

H14

S25

A 16

H17

S27

B6

B7

B8

B12

B14

B15

B16

B23

B26

B29

В30

Cl

C3

C5

ClO

C14

C15

D2

D6

D8

D15

D16

El

E2

E5

E7

E10

G3

G4

G5

G8

G9

G12

$\mathrm{H} 24$

S28

H26

J9

KI

K3

$\mathrm{KII}$

K2O

K2I

K26

I.2

L 4

L5

L6

L9

MI

MII

M14

M15

M16

M19

iN 9

02

06

07

08

P9

P2I

R3

R8

RI4

RI 7

R19

G14

G15

$\mathrm{HI}$

14

117
S31

S 32

549

S50

T3

T4

T7

T 10

TII

T12

$\mathrm{T} 13$

T15

T16

T 17

T20

T22

T23

V6

V13

W3

W5

W7

W11

WI 4

W16 
2b. Index Tabulation

Categories $10-13$

10

Al

A6

Al3

B3

B4

B15

B20

B2 4

B31

B32

C6

C19

D10

D12

D16

E3

E4

E6

F2

F7

G14

Gl6

H6

H9

HII5

H16

II

KIO

K12

L2

L15

M7

198

MIO

09

P3

$\mathrm{Pll}$

P20

P22

P 23

R19

S12

S13

528

\begin{tabular}{l} 
11 \\
\hline A14 \\
D3 \\
D13 \\
H10 \\
H1 8 \\
P3 \\
S3 \\
S30 \\
T9 \\
V5 \\
V10 \\
W11
\end{tabular}

S36

537

S38

543

S44

545

T21

W7

W12

W17

W11

10

18

S3

S30

T9

V5

10

.

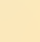

12

A4

A5

BI

B34

$\mathrm{C} 4$

Cl1

Cl6

C21

D16

F2

H4

H5

J6

K5

K7

L7

L13

L16

M18

N12

P14

R7

S20

S28

533

S34

546

547

548

S49

UI

V2

V3

V8

W. 4
13

В33

Cl7

C18

D16

I 3

$\mathrm{K} 14$

K29

N1O

P5

P13

R9

RIO

R19

S5

S6

57

58

540

W16 


\section{Index to Authors}

\section{A}

Abe, $T ., K 3$

Ablova, M.S., Al, A2

Acs, E., A3

Adlington, R.E., Gl2

Afanas'ev, V.F., M8

Airapetyants, S.V., A4

Albert, M.P., A5, C16

Allen, C.C., A6, A7

Allerton, G.L., A8, A9, A10, All

Amer, S., Al2

American Society for Testing and

Materials, Al3

Anderson, J.R., C6

Antler, M., A 14

Arai, Y., II

Armstrong, D.B., C7, C8, C9

Aron, C.P., A15

Arthur, J.B., Al6

Auletta, L.V., A14

Axelrod, S.D., W7

\section{$\underline{B}$}

Backenstoss, G., BI

Bailey, G.C., B2

Baker, D., B3, B4

Balchin, N.C., 1120

Banis, T. Ya., B5

Baranskii, P.I., B6, B7, B8

Bardeen, J., P7

Barlow, H.E.H., BlO, KI9

Barlow, H.M., B9

Barry, A.L., Bll

Bate, R.T., B12, D13

Baynham, A.C., B13.

Beach, A.L., T13

Bean, C.P., Bl4

Beer, A.C., B12, B15, D14

Bell, J.C., S28

Benanti, M., J1, J2

Benjamin, R., J1, J2, J3, J5

Bennett, D.C., Bl6

Benson, K.E., C3.

Berkova, A.V., MI4

Bhar, J.N., BI7

Biard, J.R., Bl8

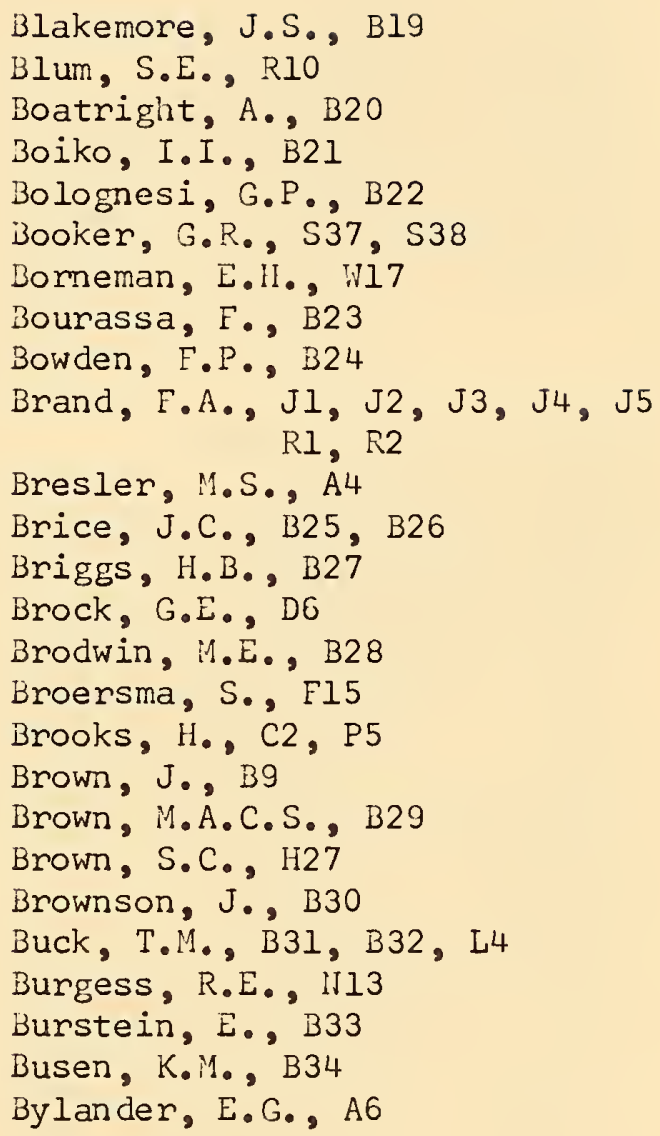

Blakemore, J.S., B19

Blum, S.E., RlO

Boatright, A., B20

Boiko, I.I., B2I

Bolognesi, G.P., B22

Booker, G.R., S37, S38

Bomeman, E.II., W17

Bourassa, F., B23

Bowden, F.P., B24

Brand, F.A., J1, J2, J3, J4, J5 $\mathrm{R} 1, \mathrm{R} 2$

Bresler, M.S., A4

Brice, J.C., B25, B26

Briggs, $H_{.} B ., B 27$

Brock, G.E., D6

Brodwin, H.E., B28

Broersma, S., F15

Brooks, H., C2, P5

Brown, J., B9

Brown, M.A.C.S., B29

Brown, S.C., $\mathrm{H} 27$

Brownson, J., B30

Buck, T.M., B31, B32, L4

Burgess, R.E., III3

Burstein, E., B33

Busen, K.M., B34

Bylander, E.G., A6

$$
\text { C }
$$

Cahn, J.H., S28

Camp, P.R., Cl

Cardona, $\mathrm{H}_{\bullet}, \mathrm{C} 2$

Carruthers, J.R., C3

Caspari, M.E., R4

Catalano, S.B., C4

Cerny, L., C5

Chaikin, S.U., $\mathrm{C} 6$

Champlin, K.S., C7, C8, C9, Cl0

Ch'ang-hou, S., Cli

Chaplin, it.J., P6

Chapman, P.W., Cl2

Chatterji, C.K., Cl3, il3, $N 4$

Chizhevskaya, S.N., G7

Chukichev, M.V., CI4 
Cihelka, J., C5

Cleland, J.W., C15

Combs, J.F., A5, Cl6

Conley, J., Al4

Conwell, E.M., C17, C18, D5, Z3

Coughlin, B.J., Cl9

Crawford, J.H., Jr., Cl5

Creamer, R.H., C2O

Crow, E.L., C2l

Csavinszky, P., C22

Cuevas, H., Fl3

\section{$\underline{D}$}

Danielson, G.C., N1l

Das, P., N1, is 5

Dash, W.C., D1, D2

Dauphinee, T.M., D3

Davies, E.A., D4

Davis, G.L., C19

DeBlois, R.W., Bl 4

Debye, P.P., D5

Denene, $M_{\bullet} V_{.}, B 5$

Dew-Hughes, P., D6

Dickey, D.H., D7, 119

Dickhoff, J.A.M., D8

Donovan, B., D9

Donovan, T.M., D10

Dorin, V.A., Dll

Dorosheski, G., K7

Dreiner, R., Dl2

Drickamer, H.G., $1117, \mathrm{SI}$

D'Stefan, D.J., K12

Duga, J.J., D13, D14

Dumas, G., D15

Dunlap, W.C., Jr., D 16

Dunsmuir, R., H25, L11

Dykman, I.1., D17, D18, D19

Dzong, T.K., Rl2

Dzyubenko, G.M., B7

\section{$\underline{E}$}

Easton, W.B., G6

Eckhardt, G., El

Edwards, D.F., E2

Edwards, J.W., E3, E4

Edwards, N.D., E5, Bll

Egli, Р.H., В3З
Eisner, R.L., E6

Ertel, A., E7

F

Fabricand, B.P., :15

Falkvik, I., Fl

Fan, H.Y., Tl

Feucht, D.L., H2O, H2l, H22

Finne, R.M., S45

Fischer, E., F2

Fischer, G., E3

Fistul', V.I., F4, F5, R18

Fivaz, R., F6

Flores, J.M., F7

Foster, L.M., K22, L9

Fowler, V.J., Z3

Frank, H., F8, F9

Frank, R.C., F10

Frei, A.H., Fll

Frenkel', V. Ya., V13

Freundlich, J.J., D13

Fritzsche, H., F12, Fl3

Fuller, C.S., F14

Furdyna, J.K., F15

Fursenko, V.D., K28

Furukawa, Y., Fl6

G

Gardner, E.E., GI

Garnache, R., D12

Gurtner, W.W., G2

Geballe, T.H., P16, P17

Georges, C., D15

Gergely, Gy., G3

Gibbs, W.E.K., G4

Gibson, A.F., A16, B13, G5

Gilman, J.J., W10

Glang, R., G6

Glazov, V.M., G7

Glazunov, P. Ya., V9

Goetzberger, A., G8, H1, S23

Goldey, J.M., Hi27

Golovin, B.I., 115

Good, R.H., Jr., L12

Goorissen, J., G9

Gorid'ko, i. Ya., Glo

Gorkun, Yu. I., Gll 
Gosling, D.S., D4

Goss, A.J., Gl2

Gossenberger, H.F., MlO

Gossick, B.R., Gl3

Gould, H.G., Gl4

Grandjean, C., G15

Grantham, L.F.,. YI

Granville, J.W., Al6, Bl3

Greenwood, J.A., Gl6

Greig, D., F3

Gubenko, A. Ya., F4

Guldbrandsen, T., M12

Guldner, W.G., T13

Gunderson, P.D., C9

Gunn, M.H., G17, G18

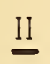

Helgglund, J., HIl

Haitz, R.H., G8, Hl

Hall, G.L., H2

Hallenback, J.F., Jr., Gl, S9, Slo

Hamer, H., 113

Hanamura, E., T2

Hansen, $\mathrm{L} . \mathrm{B} ., \mathrm{H} 4$

Hantay, 0., 63

Hargreaves, J.K., H5

Harman, G. . ., H6, H15

Harman, T.C., H7

Harrick, N.J., H8, 119

Harris, P.T., T 16

Haupin, W.E., H10

Hedvall, P., Hll

Heinecke, W.J., 1

Heitz, A., Wl2

Hellinger, O., Nlo

Helpert, E., MlO

Henisch, H.K., H12

Herring, C., H13

Heywang, W., $\mathrm{Hil} 4$

Higier, T., H6, H15

Hillegas, W.J., Jr., HI6

Hirshon, J.M., Hl7

Hitchcox, G., Hl8

Hoffman, A., H19

Holmes, D.A., H2O, H2l, H22, J5

Hora, $\mathrm{H}_{\bullet}, \mathrm{H} 23$

Horn, F.H., H24

Horner, F., 1125
Hornstra, J., Ii26

IIsieh, H., H27

Husa, (Gusa) V., C5, 1128, K27

I

Ida, I., II

Il'ina, $1 . A .$, I2

Ioffe, A.F., I3

Irvin, J.C., I 4

Isaacs, J., 02

Ittner, W.B. III, I5

Ivko, V.1., S3

\section{J}

Jackson, H., H25

Jacobs, H., H22, J1, J2, J3, J4, $\mathrm{J} 5, \mathrm{R} 1, \mathrm{R} 2$

Jacobson, A.H., Jr., J6

Jaggi, R., J7

Johnson, W.E., J8

Jones, A.H., D6

Jorgensen, $\mathrm{M} .11 ., \mathrm{J} 9$

$\underline{K}$

Kahng, D., Kl, T8

Kalvenas, S.P., K2

Karstensen, F., 69

Kato, $\mathrm{H}_{0}, \mathrm{~K} 3$

Keller, J.B., $K 4$

Kennedy, D.P., K5

Kennedy, J.K., K6

Keywell, F., K7

Khotkevich, V.I., K8, K9

Kingsnorth, R.L., C19

Kirvalidze, I.D., KlO, Kll

Klein, D.L., KI2, $\$ 45$

Klein, M.A., K23

Klinger, M.I., $\mathrm{Kl} 3$

Knight, G., Jr., K14

$\mathrm{Ko}, \mathrm{N}_{-}-\mathrm{H} ., \mathrm{K} 15$

Kobayashi, M., T2

Koenig, S.H., Kl6

Kohane, T., K17, Kl8

Koike, R., Kl9

Kokorev, D.T., K20

Kolb, E.D., K2l 
Kolb, G.A., S45

Konoplyasova, N.S., B7

Kovtonyuk, N.F., K20

Kozak, M.I., ZI

Kozlov, M.M., DII

Kramer, R.A., K22

Kressel, H., K23

Krishna, K.V.G., BlO

Kroemer, H., K24

Krongard, R.R., ClO

Ksoll, G., K25

Kudman, I., K26

Kurilo, P.H., B8

Kurova, I.A., I2

Kutsko, 11., Llo

Kuz'menko, P.P., Glo

Kvasil, B., $\mathrm{H} 28, \mathrm{~K} 27$

Kynev, St., K28

Kytoniemi, T., K29

\section{$\underline{L}$}

Labuda, E.F., Ll

LaChapelle, T.J., L2

Lamb, J , H25

Lamorte, M.F., L3

Lander, J.J., L4

Landwehr, G., L5

Lang, A.R., L6

LaP Iume, J., L7

Lark-Horovitz, K., J8

Larrabee, R.D., L8

Leboeuf, M.B., T10

LeCraw, R.C., LI

Lederhandler, S.R., El

Lewis, J.E., L9

Liben, W., PI5

Lindmayer, J., L10

Linhart, J.G., LII

Liu, S.H., Ll2

Logan, M. A., LI3

Logan, R.A., FI4

Long, D., Cl2, L14

Lorinczy, A., Ll5

Love, E.R., Ll6

Luchsinger, E.B., Yl

$M$

MacDonald, A.L., MI, M2

Machlup, S., M13
Maczuk, J., M3, M4, M5

Magill, P.J., I5

Maita, J.P., M22, 1123

Maker, P.D., E2

Many, A., 06, 07, 08

Manz, R.C., Kl, T8

llarch, i.tl., D3

Martinuzzi, S., 116

Maruyama, S., M7

Maslova, L.V., M8

Matare, H.F., R8

Mathews, J.R., L4

ilatveev, O.A., :18

hazur, R.G., 149

McDonald, B., G8

Uckean, W.J., P18

HcKim, F.S., B32

Uciamara, J.E., W12

Mehl, W., MlO

Meindl, J., JI, J2, J3, J4, J5

Meltzer, G., Mll

Merkl, N.A., B20

llette, 1I., B20

lleyer, iN.I., J9, 1112

Michels, A., 1113

Millard, D., 15

Mills, A.D., T4

Mil'vidskii, M.G., M14, M15, T23

Minden, H.T., MI6

Minomura, S., $M 17$

Mircea, A., M18

Miselyuk, E.G., VIl

Mizuguchi, K., S4

Mlavsky, A.I., W7

Montgomery, H.C., M19

Moore, P., B25

Hooser, E., F3

Mordike, B.L., M20

Morgan, T.i.., 1121

Morin, F.J., M22, M23

Morozov, G.P., P8

Motchenbacher, C.D., L14

Muraoka, H., K3

Myers, J., LI4

N

Nag, B.R., Cl3, il, iv2, iN $3, \mathrm{~N} 4, \mathrm{i} 5$ Nakamura, M., N6

Nakhodkin, i. G., N7

ivemeth, T., LI5 
Nemtsev, V.P., N7

Nesbitt, L.B., B14

Neuberger, M., N8

Newkirk, J.B., N9

Nibler, F., N10

Nishina, $\mathrm{Y}_{.}, \mathrm{L} 12, \mathrm{Nll}$

Nomura, $\mathrm{Y}_{\bullet}, \mathrm{N} 12$

Novikov, N.N., Glo

Nyberg, D.W., N13

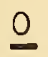

Ogawa, T., 01

Okada, O., M7

Okkerse, B., G9

Oldfield, W.W., Jr., S29

Oliver, C.R., 02

Olshefski, P.J., 03, 04

Omel'yanovskii, E.M., F5

Oroshnik, J., 05, 06, 07, 08

Ostapkovich, P.L., O9

\section{$\underline{P}$}

Pagnia, H., Pl

Paige, E.G.S., Al6, G5

Pankey, T., P2

Pany, F.G., P3

Patskevich, V.M., V9

Paul, W., C2, P4, P5

Paulnack, C.L., P6, S27

Pearson, G.L., P7

Peat, A.J., Wll

Petrusevich, V.A., P8

Pfann, W.G., P9, P10

Pharo, W.B., Pll

Pierno, A., B22

P'i-huang, C., P12

Pistoulet, $\mathrm{B}_{\text {., }} \mathrm{P} 13$

Plakida, iv.M., P14

Poehler, T.0., P15

Pollak, M., P16, P 17

Poltinnikov, S.A., S42

Pompliano, L.A., S45

Potter, R.F., Pl8

Powell, R. C., P19

Pozhela, Yu.K., B5, K2

Presnov, V.A., P20

Price, P.J., P2I

Pugh, E.N., P22, P23
Ramsa, A.P., RI, R2

Ranaldi, M., 09

Ransom, P., R3

Rashba, E.I., B2I

Rasmussen, A.L., P19

Rau, R.R., R4

Ravich, Yu.I., R5

Rawlins, T.G.R., R6

Reber, J.M., R7

Redaelli, G., B22

Reed, B., R8

Regel, A.R., A2

Reid, F.J., R9, R10

Reuschel, K., H19

Reynolds, J.E., RIl

Richter, G., R12

Rindner, W., RI3

Roberts, D.H., RI4

RoDin, J., RI5

Rose, K., Rl6

Rothberg, M., W6

Rouse, R.L., RI7

Roy, S.K., Cl3, N2, N3, N4, N5

Rubinshtein, R.N., Rl8

Rudenberg, H.G., R19

Runyan, i.R., A7

Rupprecht, H., H19

Ryan, F.M., R2O

Ryuzan, O., R21

S

Samara, G.A., SI

Samuels, L.E., P22, P23

Sandus, $0 .$, S2

Santos, G.J., Jr., C6

Sapogin, L.G., S3

Sasaki, H., N6, 54

Sawyer, B., B16

Scarlett, R.M., G8, HI

Scheibner, E.J., S5, S6, S7, S8

Scinmidt-Tiedemann, K.J., J9

Schnable, G.L., H16

Schreiber, H., L4

Schumann, P.A., Jr., Gl, S9, S10

Sll

Schwabe, G., S12 
Schwuttke, G.II., S13, S14, S15, S16, S17

Seeger, A., S18, S19

Seifert, J.R., A8, A9, Al0, All

Selikson, B., 520

Seraphin, B.O., D10

Seybolt, A.U.,

Sharpe, C.B., S2l

Sheiner, L.S., SII

Sheinkman, M.K., K28

Shelykh, A.I., S22

Shibuya, M., $S 4$

Shirn, G.A., B34

Shockley, H., Hl, S23

Shou-wu, W., S24

Shul'ga, I.B., K28

Shuvalov, Yu. N., S25

Sidyakin, V.G., S26

Silverman, S.J., S27

Simon, R., S28

Sirvetz, M.H., K18, S29

Skorik, E.T., S26

Smith, K.D., V3

Smith, M.J.A., S30

Smith, R.C., S31, S32

Smits, F.1., S33

Smythe, W.R., S34

Snodgrass, R.J., S35

Soffa, A., Wl

Soled, J., $\$ 12$

Spitzer, H.G., S36

Statz, H., S19

Stearns, C.A., M2

Stickler, R., S37, S38

Strause, B.M., T10

Stride, A.A., B26

Strutt, M.J.O., Fll

Stubb, T., S39

Stuttard, B., S40

Subashiev, V.K., P8, S4l

Subashchiev, V.K., S42

Sullivan, M.V., S43, S44, S45

Sunde, E.D., S46

Swartzendruber, L.J., S47, S48, 549

Swiderski, J., S50

Synorov, V.F., P20

Szebeni, P., L15

\section{$\underline{T}$}

Tanaka, S., Tl, T2

Tanenbaum, M., K21, S36, T3, T4

Tarnick, U., T5

Tarui, Y., T6

Tauc, J., T7

Taylor, T.A., H25

Templeton, I.M., LII

Ten Seldam, C.A., MI3

Thomas, C.O., Kl, T8

Thomas, J.L., T9

Thompson, B.A., Tlo

Thornton, F.D., Tll

Thornton, P.R., I 12

Thurmond, C.D., TI3

Tillman, J.R., 35

Tomchuk, P.M., D17, D18, D19, T14

Tomono, M., TI5

Trainor, A., Tlo

Tramposch, R.F., RI3

Trofimenko, A.P., B2I

Trumbore, F.A., T17

Tufte, O.iN., C12, T18, T19

Tulk, A.S., T20

Turner, D.R., T21

Turovskii, B.M., T22, T23

Tzoar, i., T24

$\underline{U}$

Uchinokura, K., T2

Uhlir, A., Jr., Ul

V

Valdes, L.3., V1, V2, V3

Van der Pauw, L.J., ${ }_{\text {V6 }}^{H 26}, V_{4}, V 5$,

Van Eck, J., $M 13$

Vasil'yev, G.F., V7

Vaughan, D.E., V8

Vavilov, V.S., C14, V3

Verbra, A.I., 35

Verma, J.K.D., V10

Vernon, R.J., B28

Vinetskii, R.M., Vll

Vodop'yanov, L. K., V12

Volchock, B. A., V13 
Wagrez, P., $\mathrm{WI}$

Ward, A.L., W2

Wasscher, J.D., W3

Watelski, S.B., B18

Weaver, F. W. , 14

Webb, J.K., 15

Weingarten, I. R., N6

Weinreich, O.A., R.8

Weinstein, $M_{0}, W 7$

Weisberg, L.R., W8

Weitz, S., J4, J5

Wenner, F., 19

Wernick, J.11., it9

Westbrook, J.il., W10, W1l

Whitten, W.i丁., Jr., W12

Wike, R., Yl

Willardson, R.K., D14, R10

Villiams, C.14., B2

Williamson, J.B.P., B24, G16

Wilson, B.L.H., RI4

Wintenberger, M., W13, W14

Wolfe, G.H., W15

Woods, J.F., W16

Wurst, E.C., Jr., W17
Yosim, S.J., YI

Yurkov, B. Ya., Vg

$\underline{Z}$

Zabara, M. Ya., K8, K3

Zerbst, M., HI 4

Zhukov, V.F., Klo, Kll

Zhuravlev, V.A., ZI

Zimmerman, J.E., Z2

Zook, J.D., Cl2

Zucker, J., H12, Z3

4. Bibliography

The bibliography is presented on the following pages.

The assistance provided by members of the Electron Devices Section, especially Barbara Averitt, Frank H. Brewer and Lydon J. Swartzendruher, in the preparation of the material for this Technical Note is gratefully acknow ledged. 


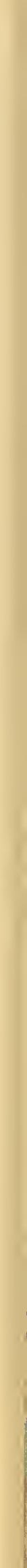



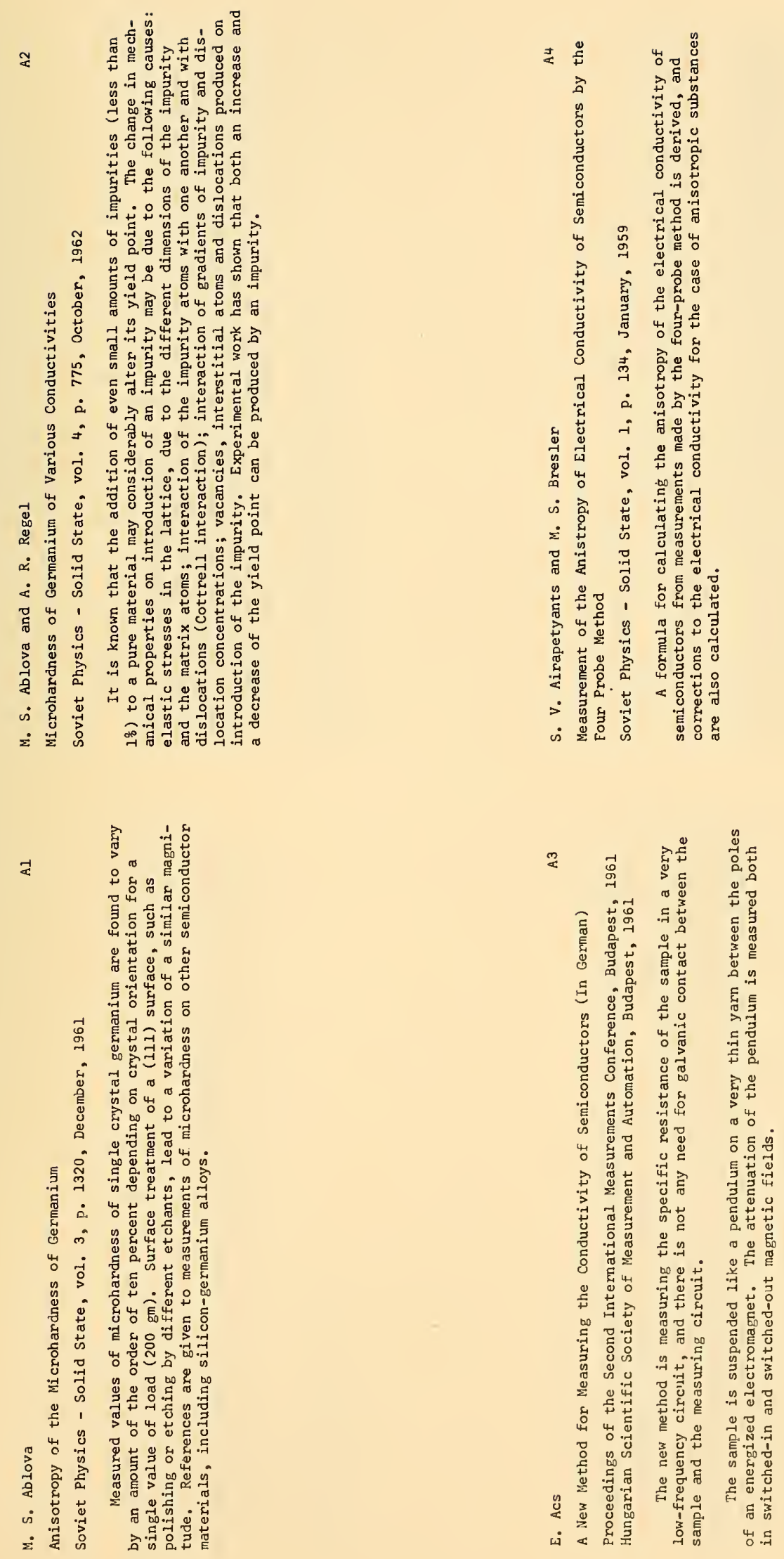

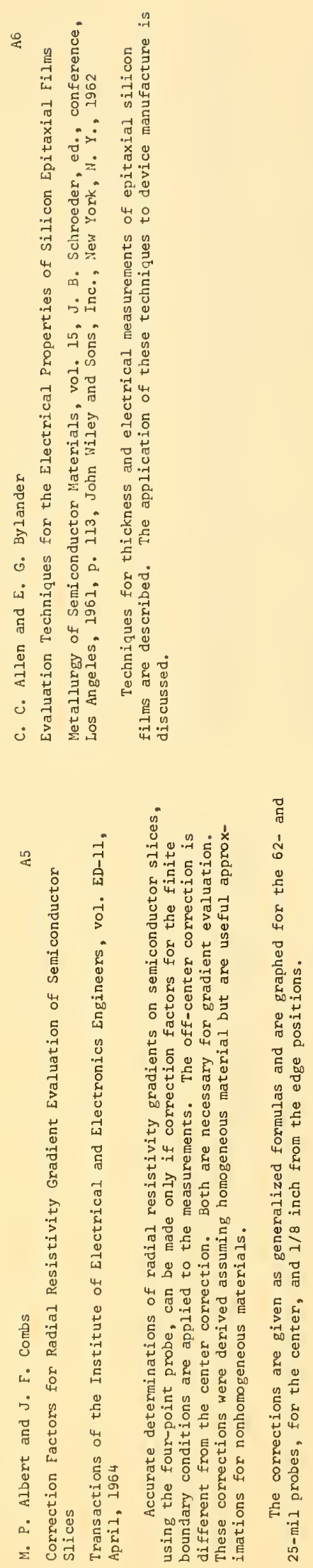
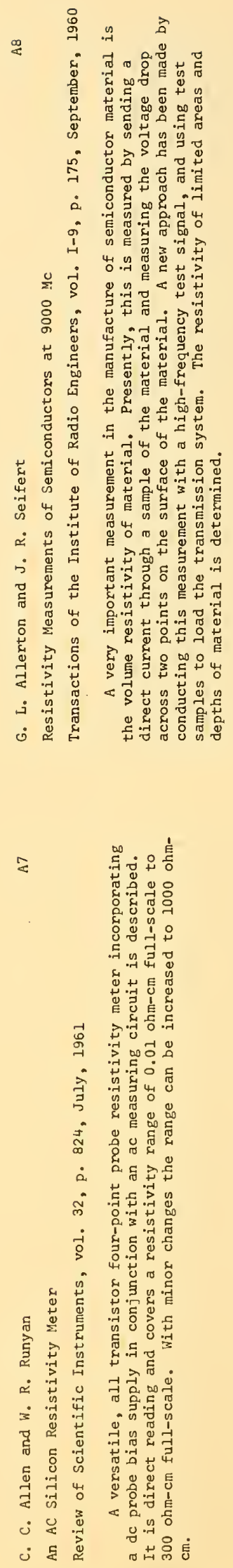

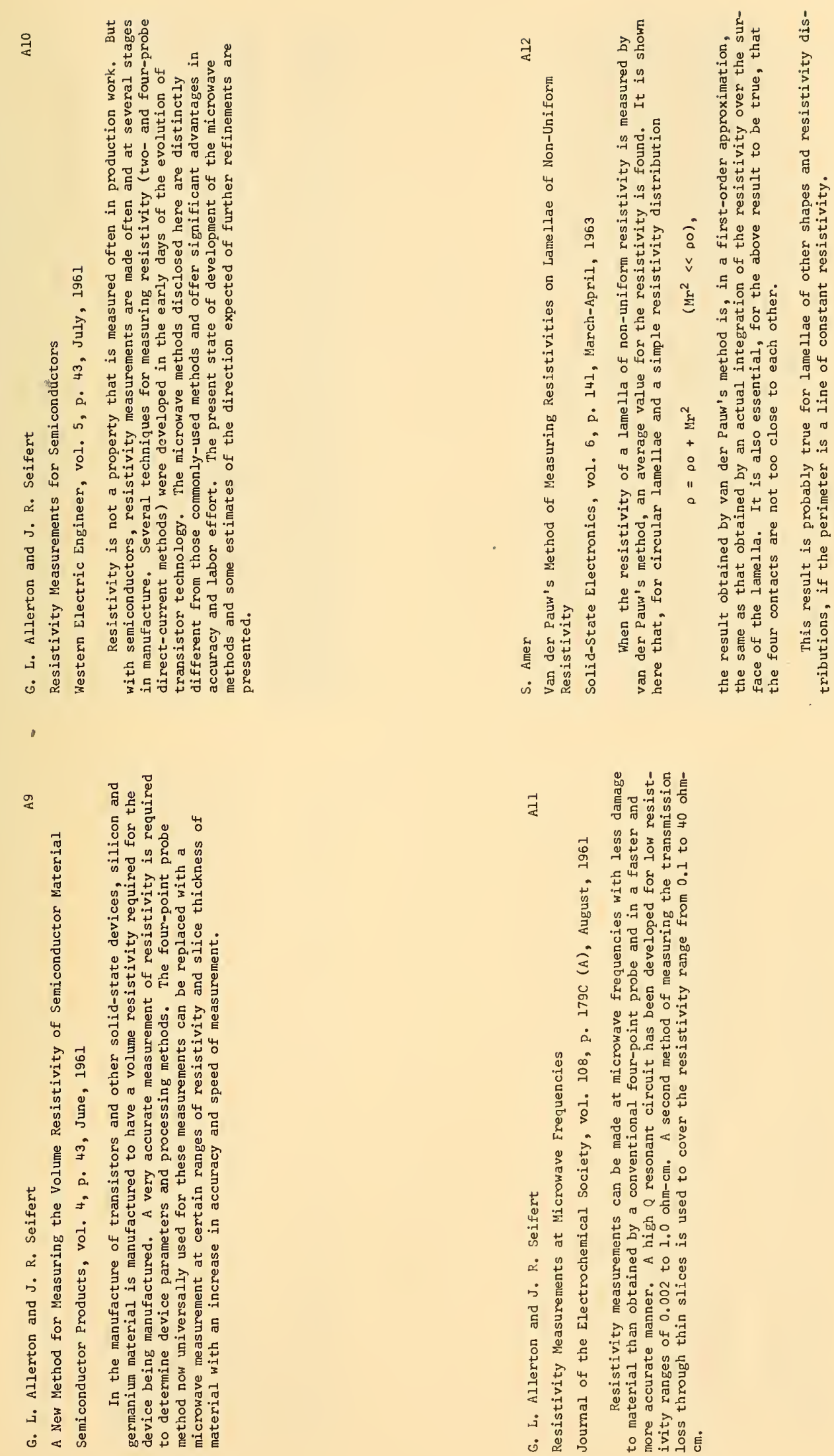

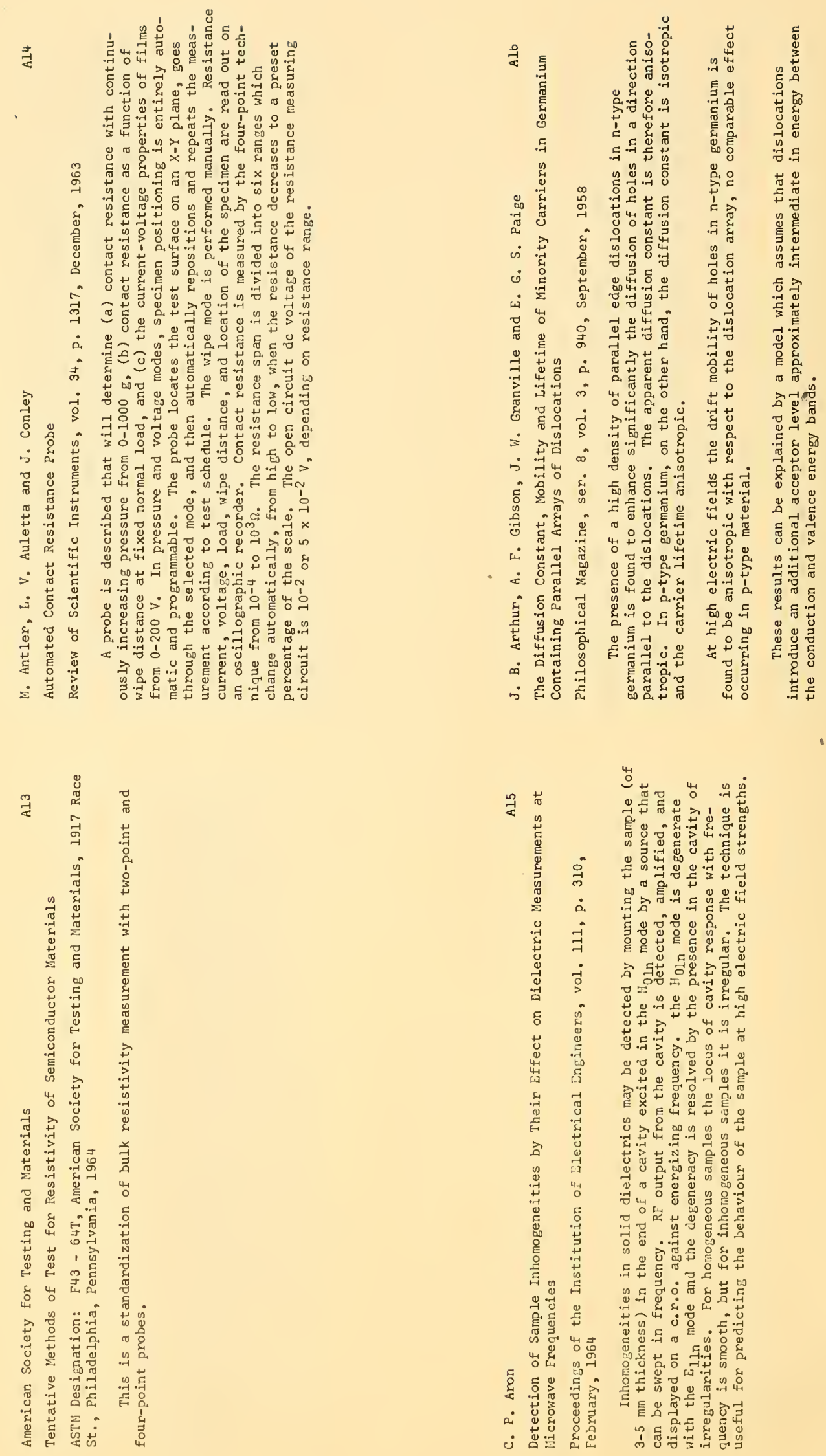

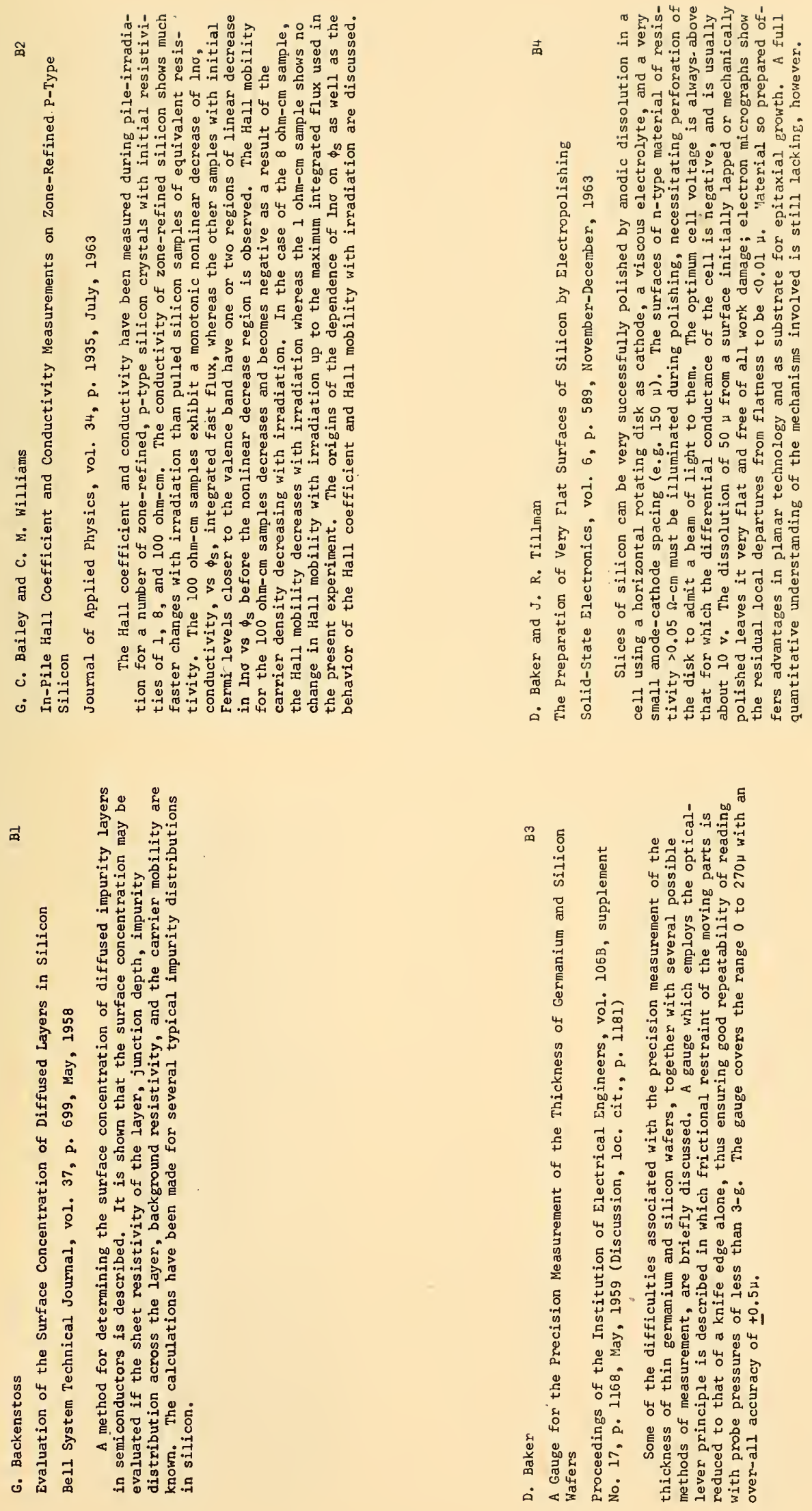

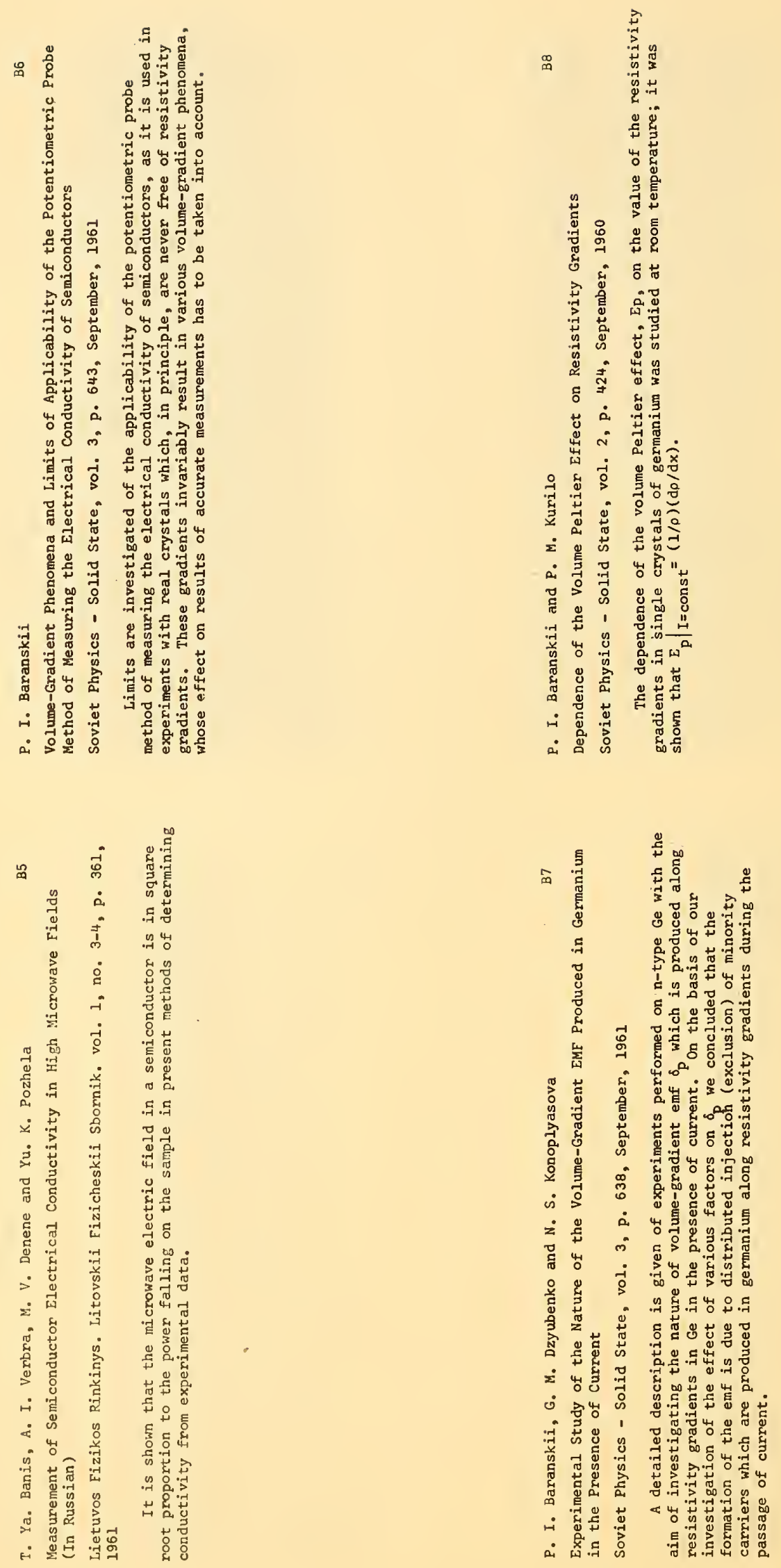


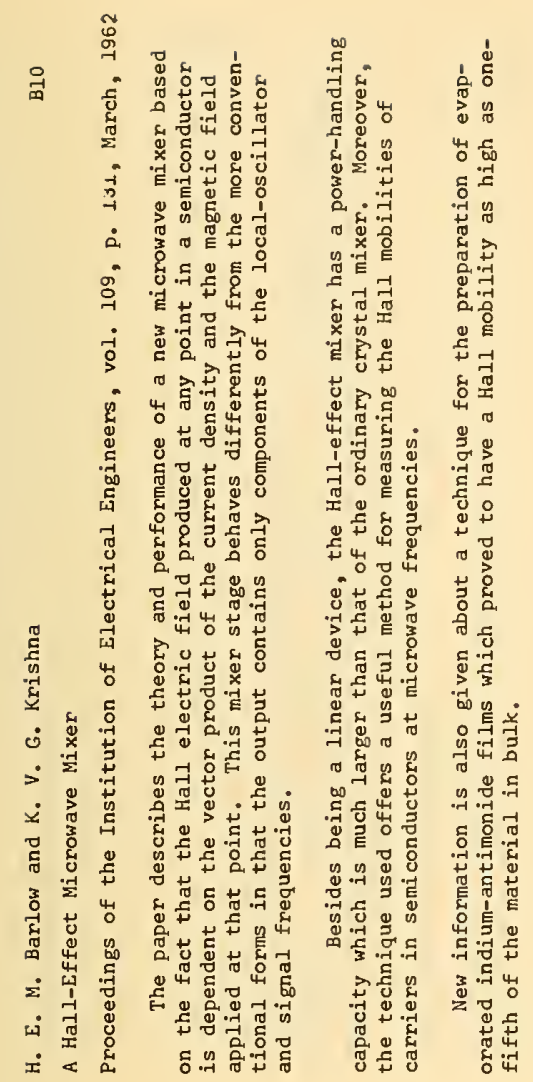

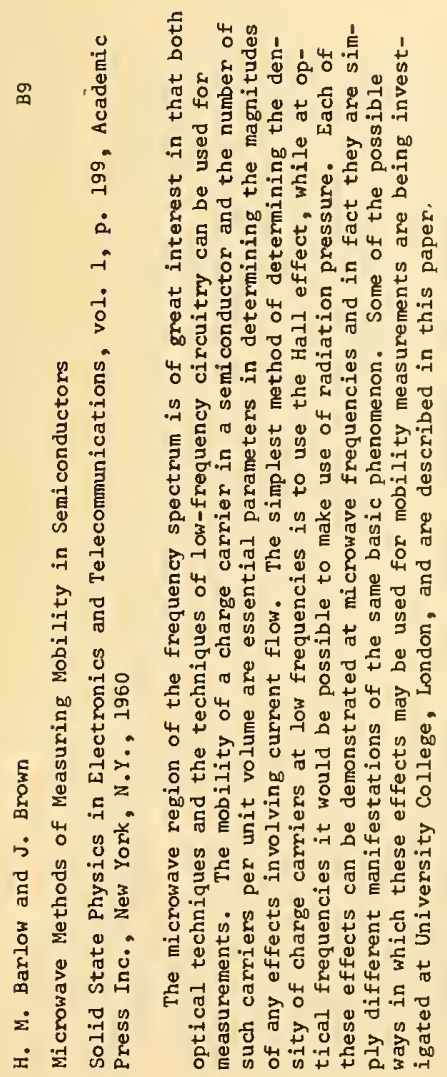

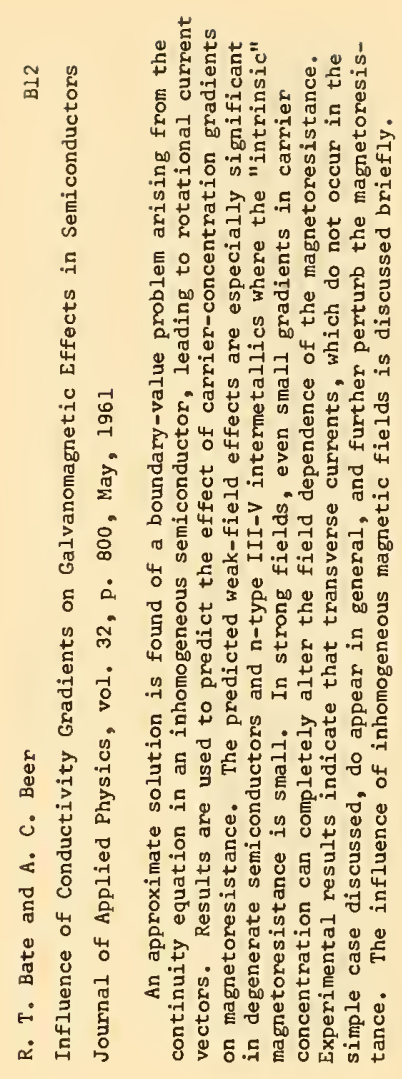

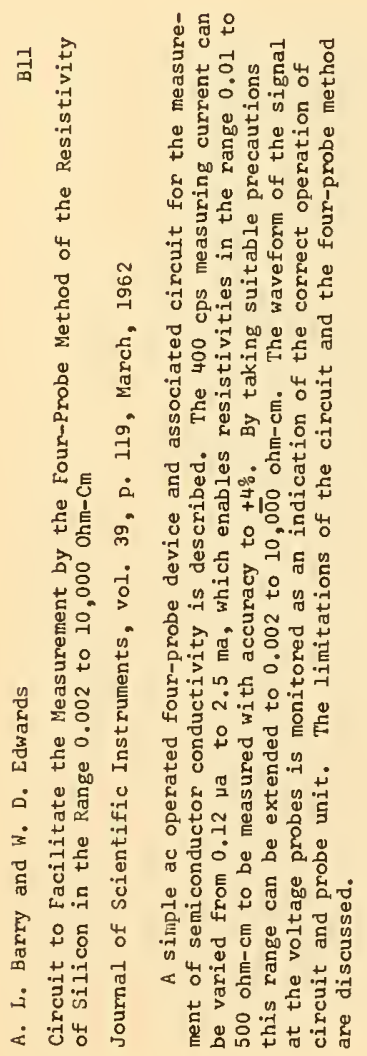



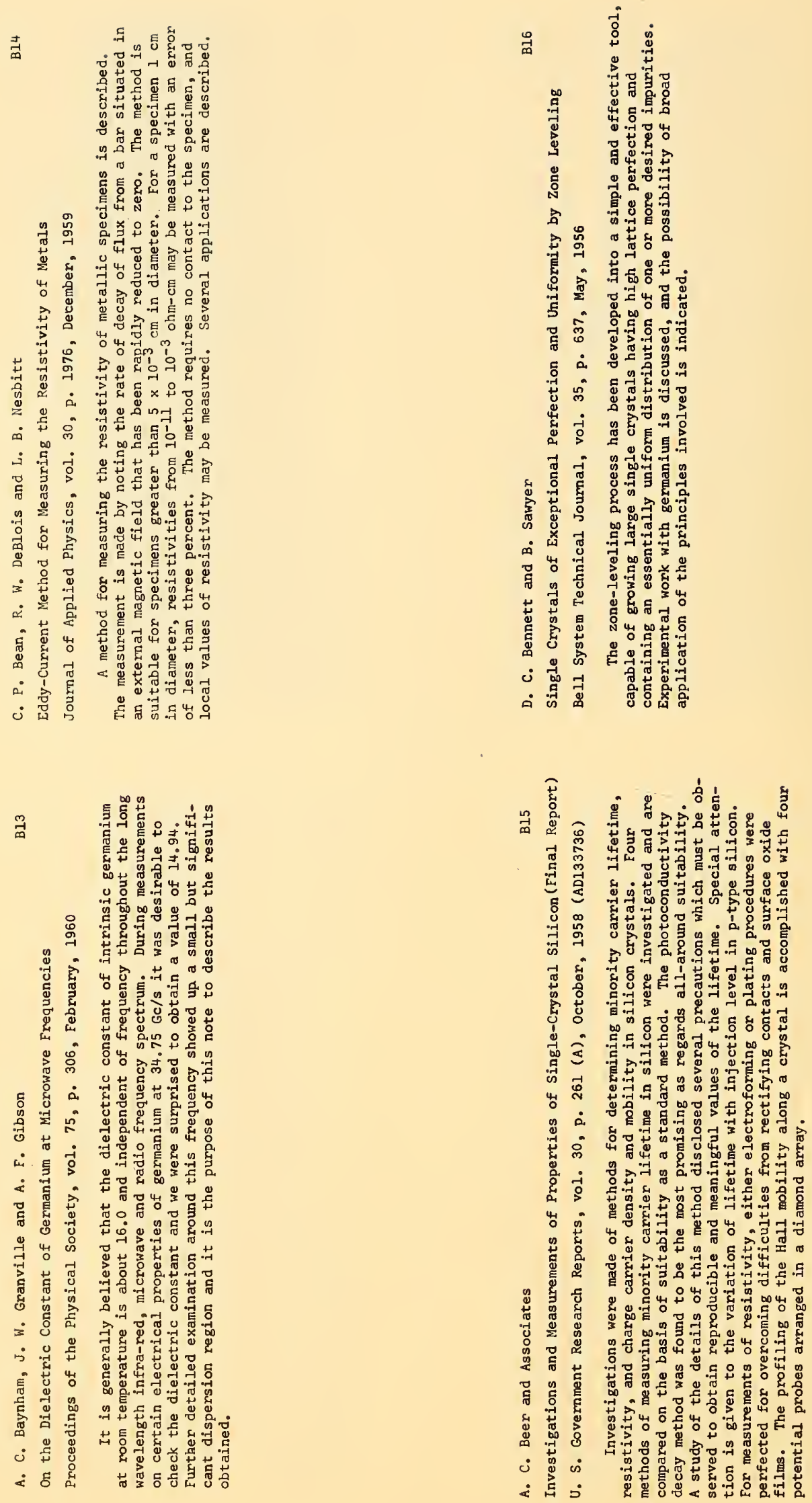


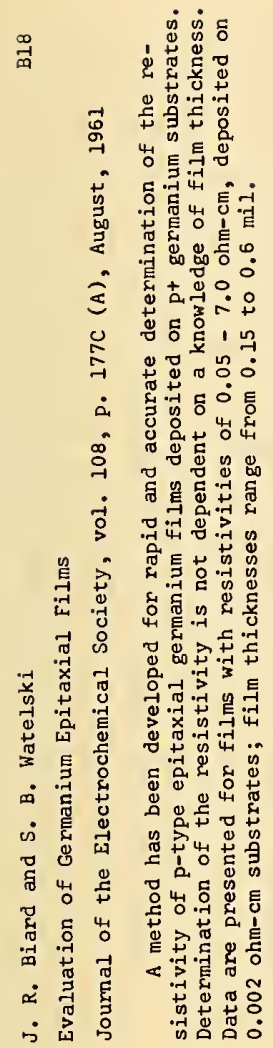

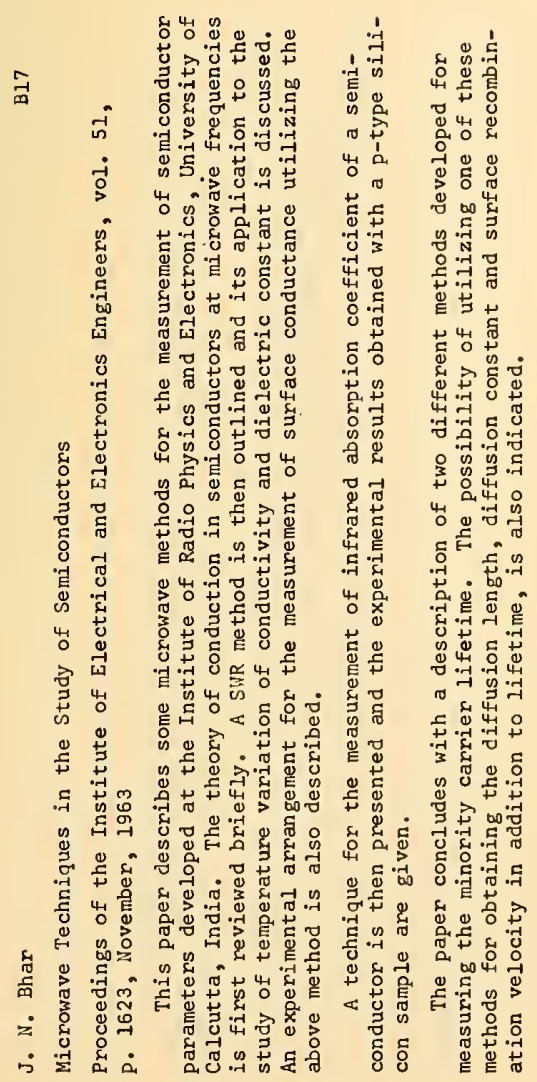
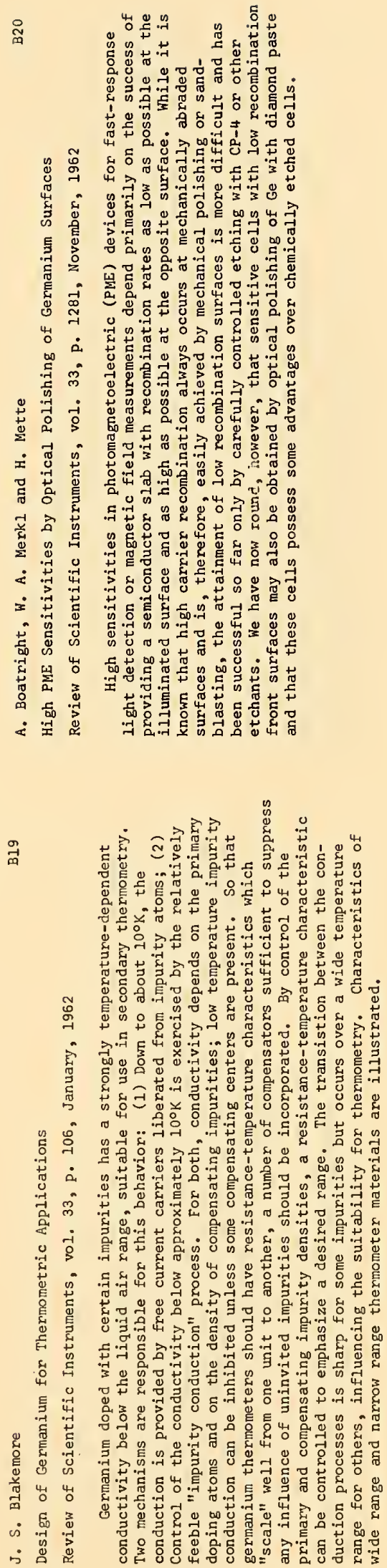

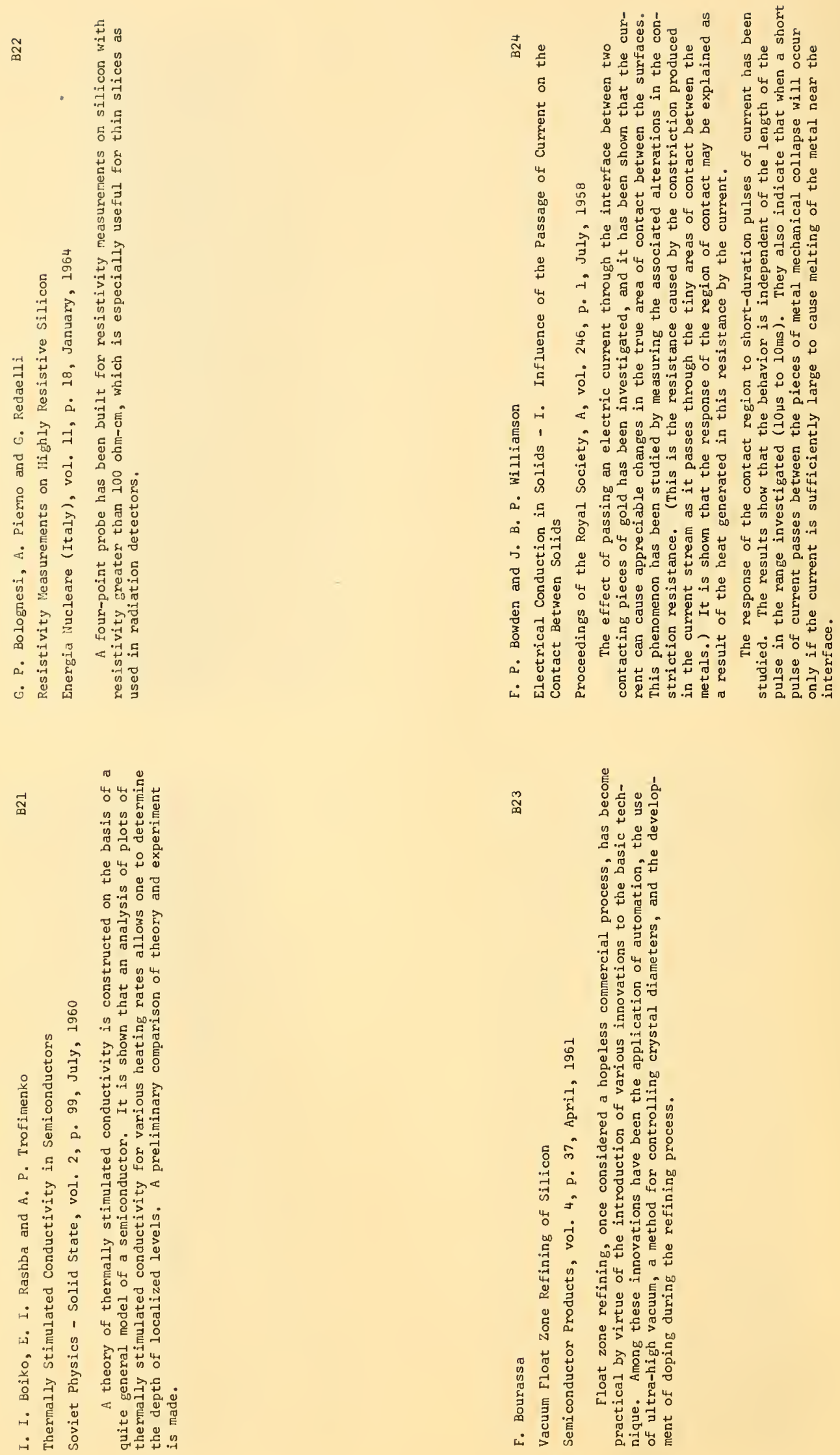

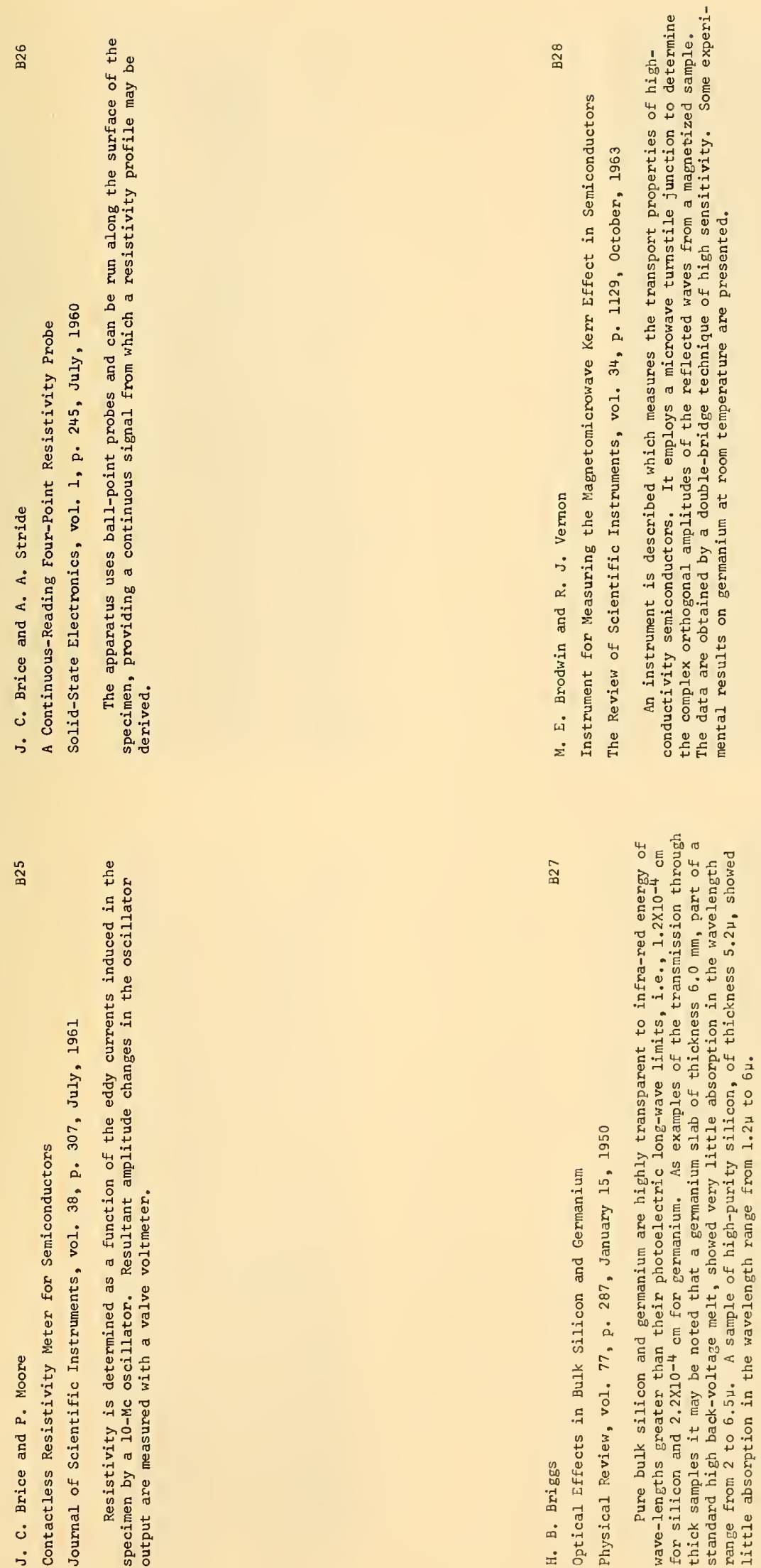

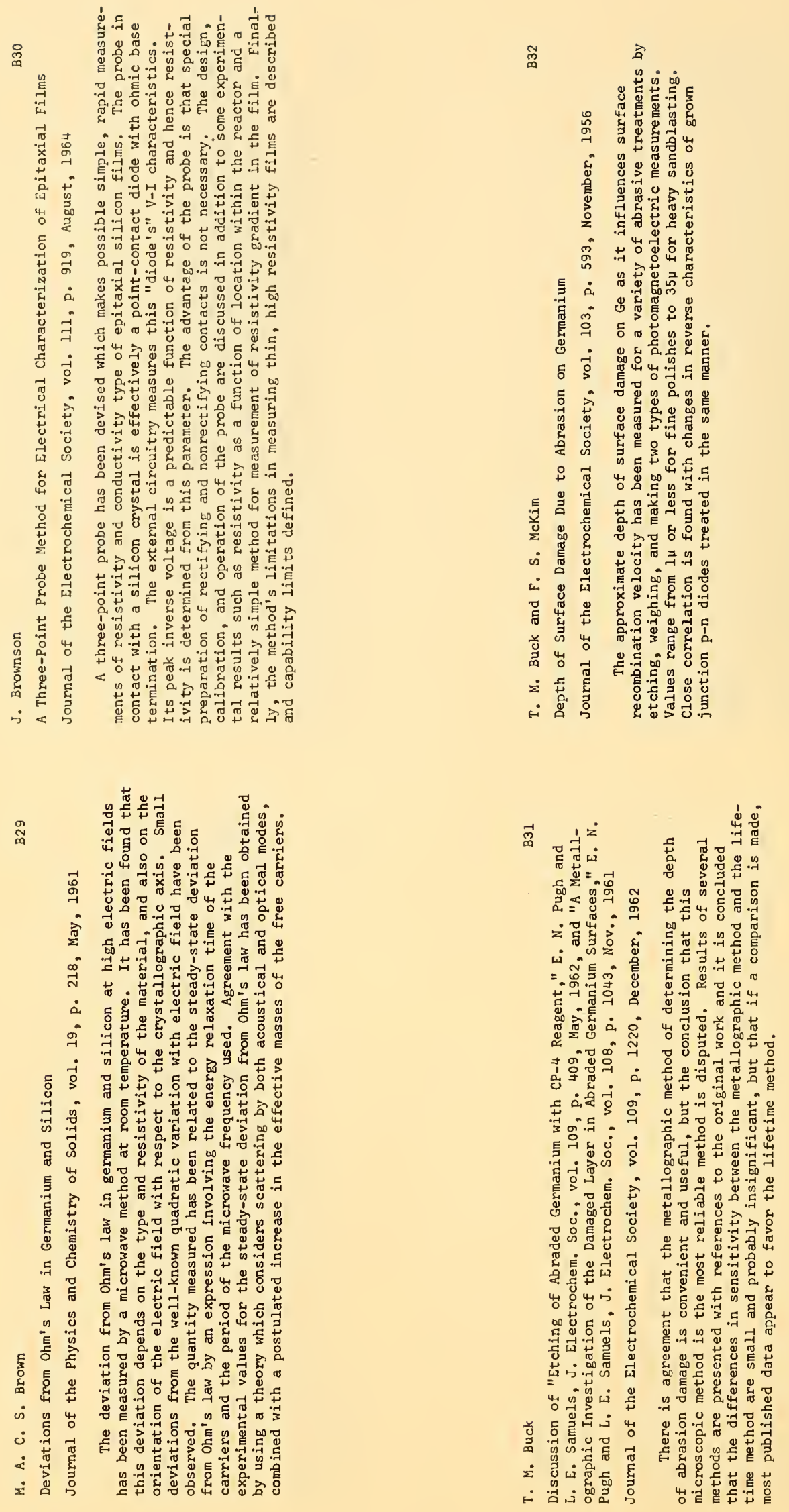

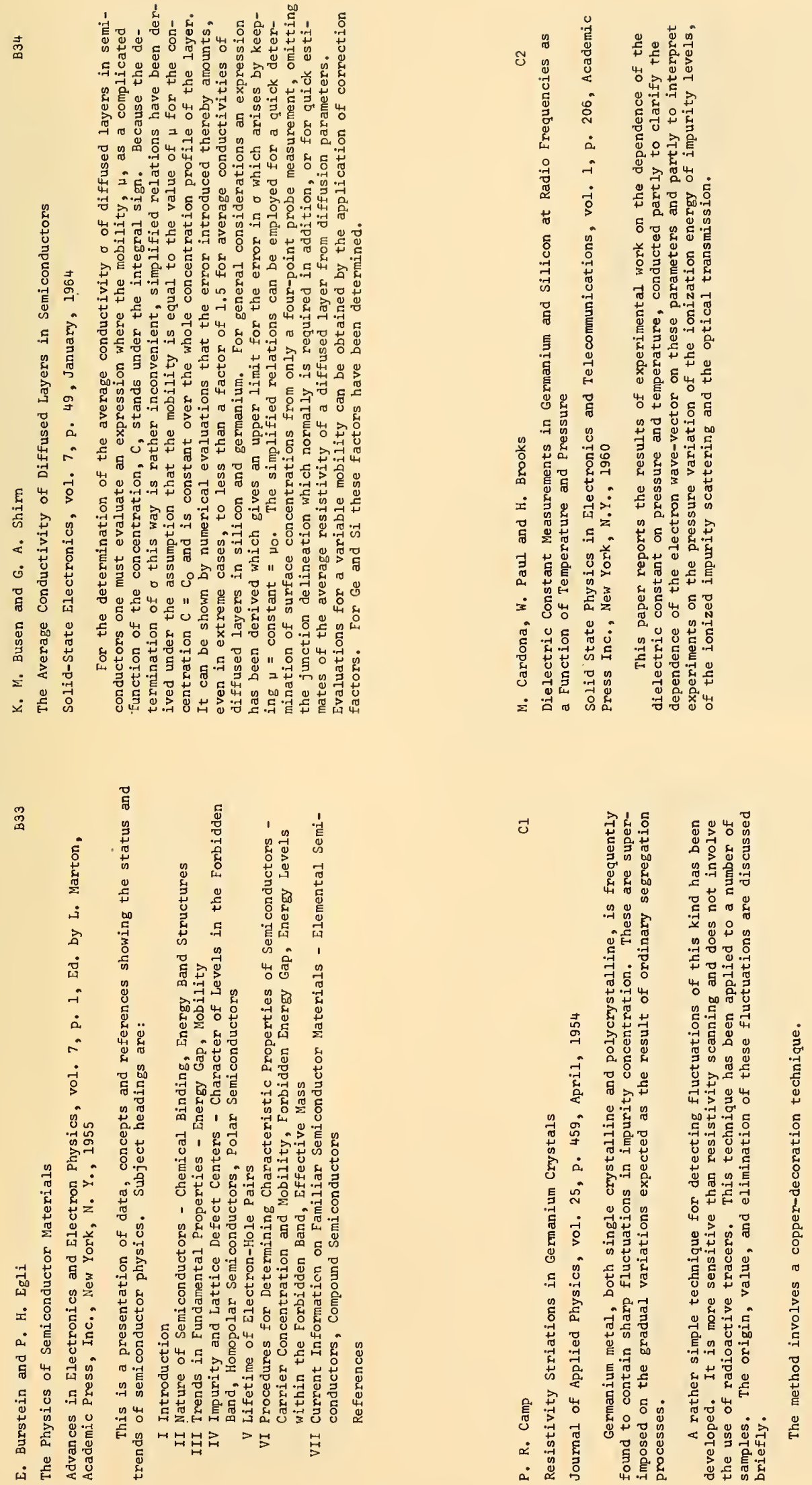

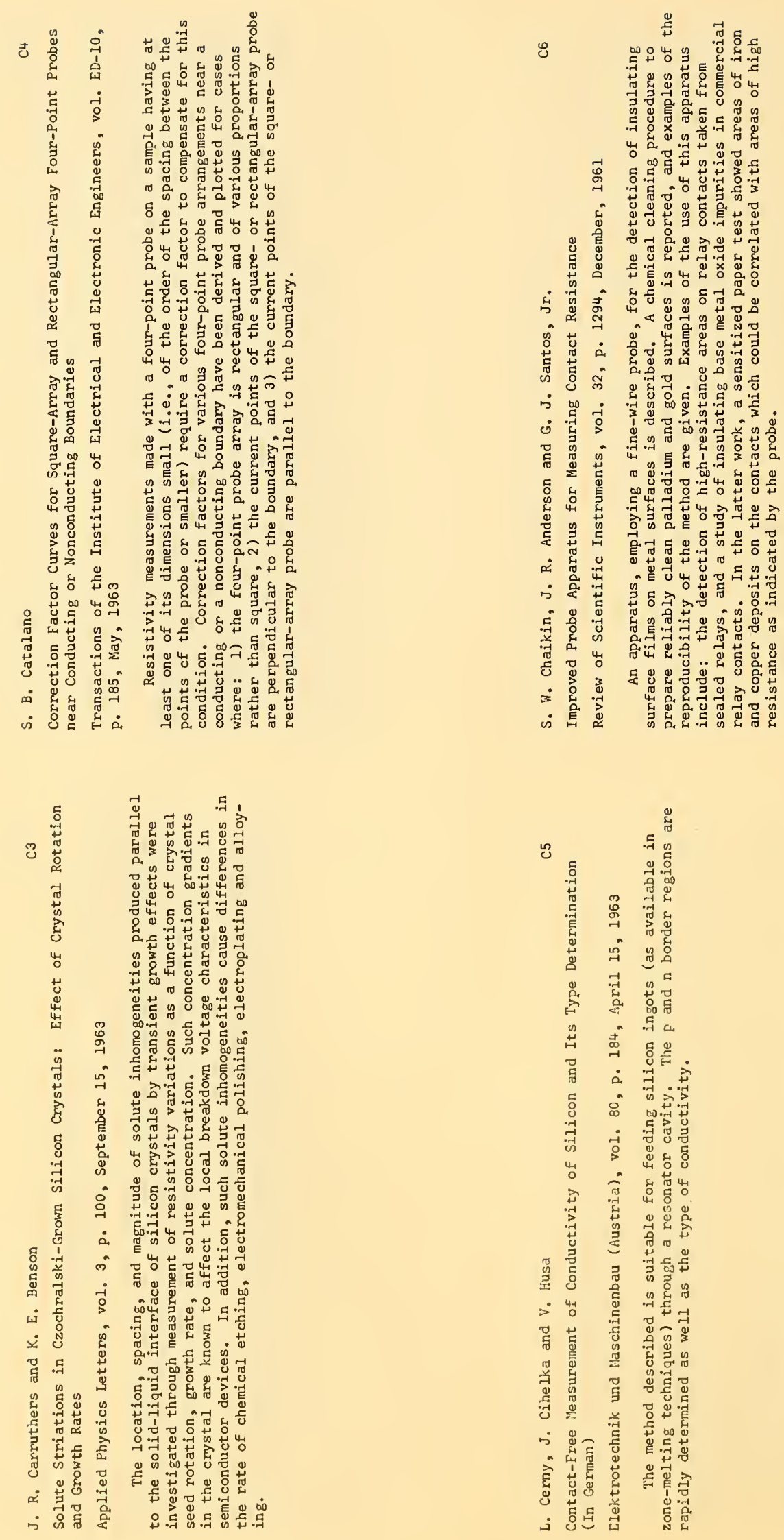

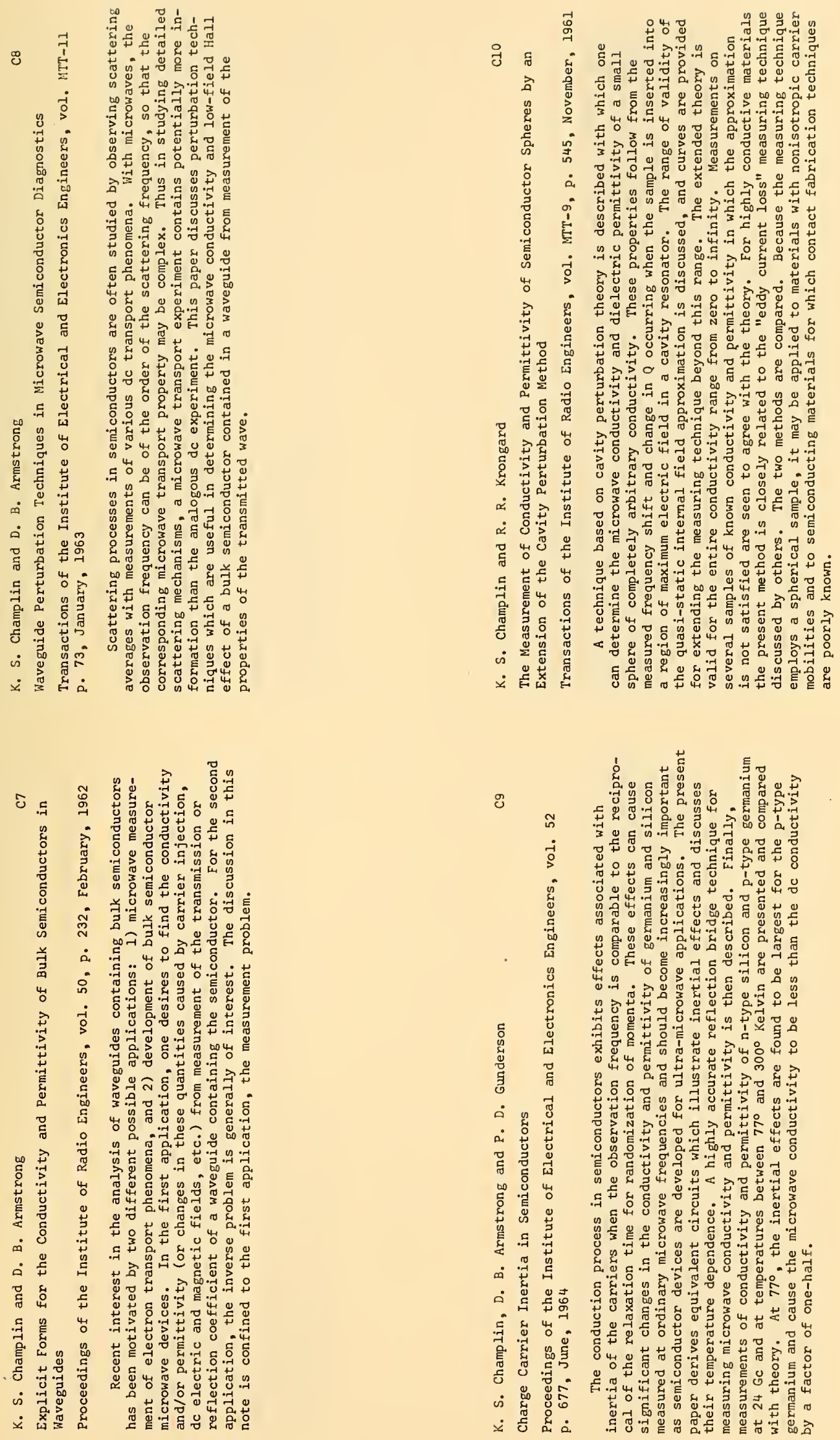

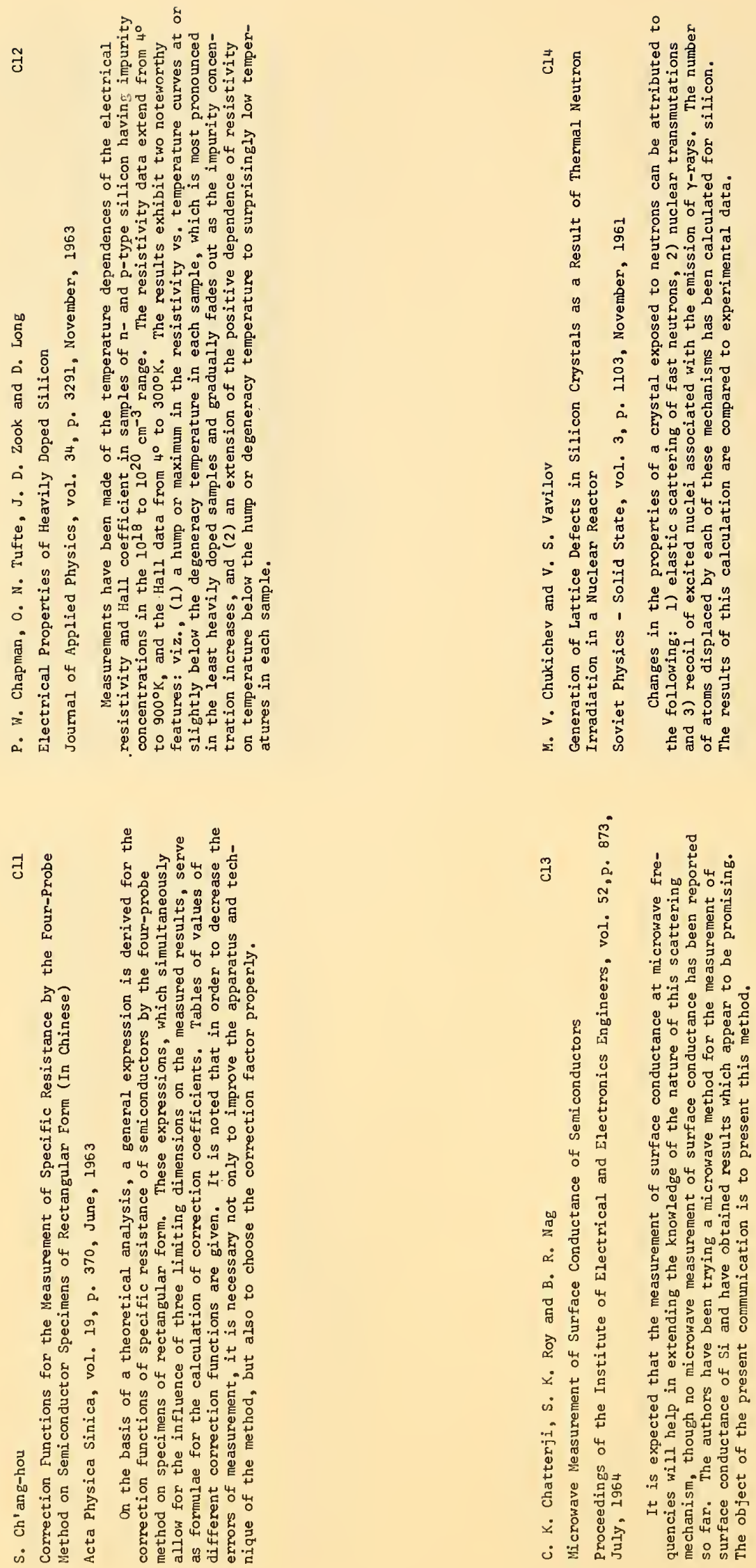

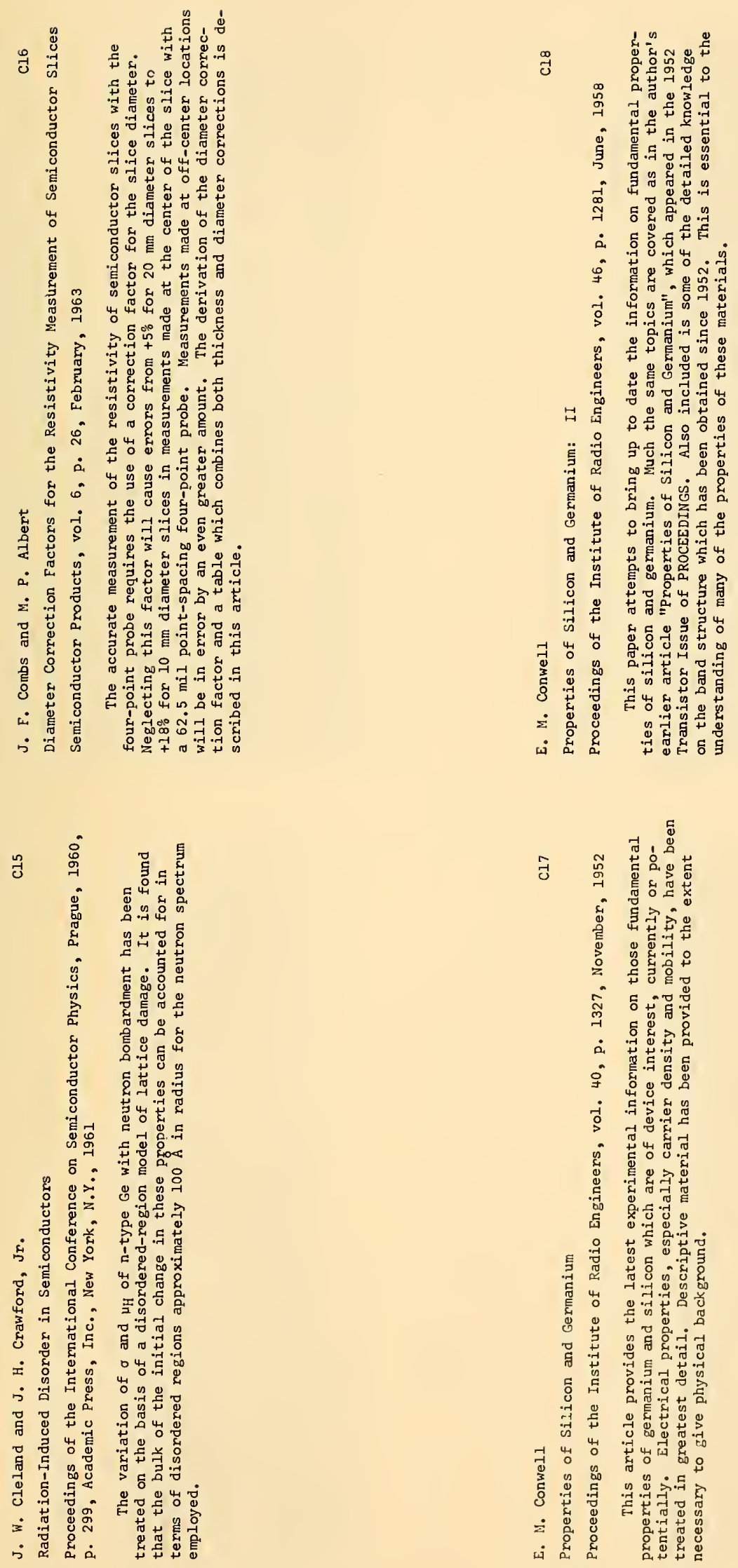

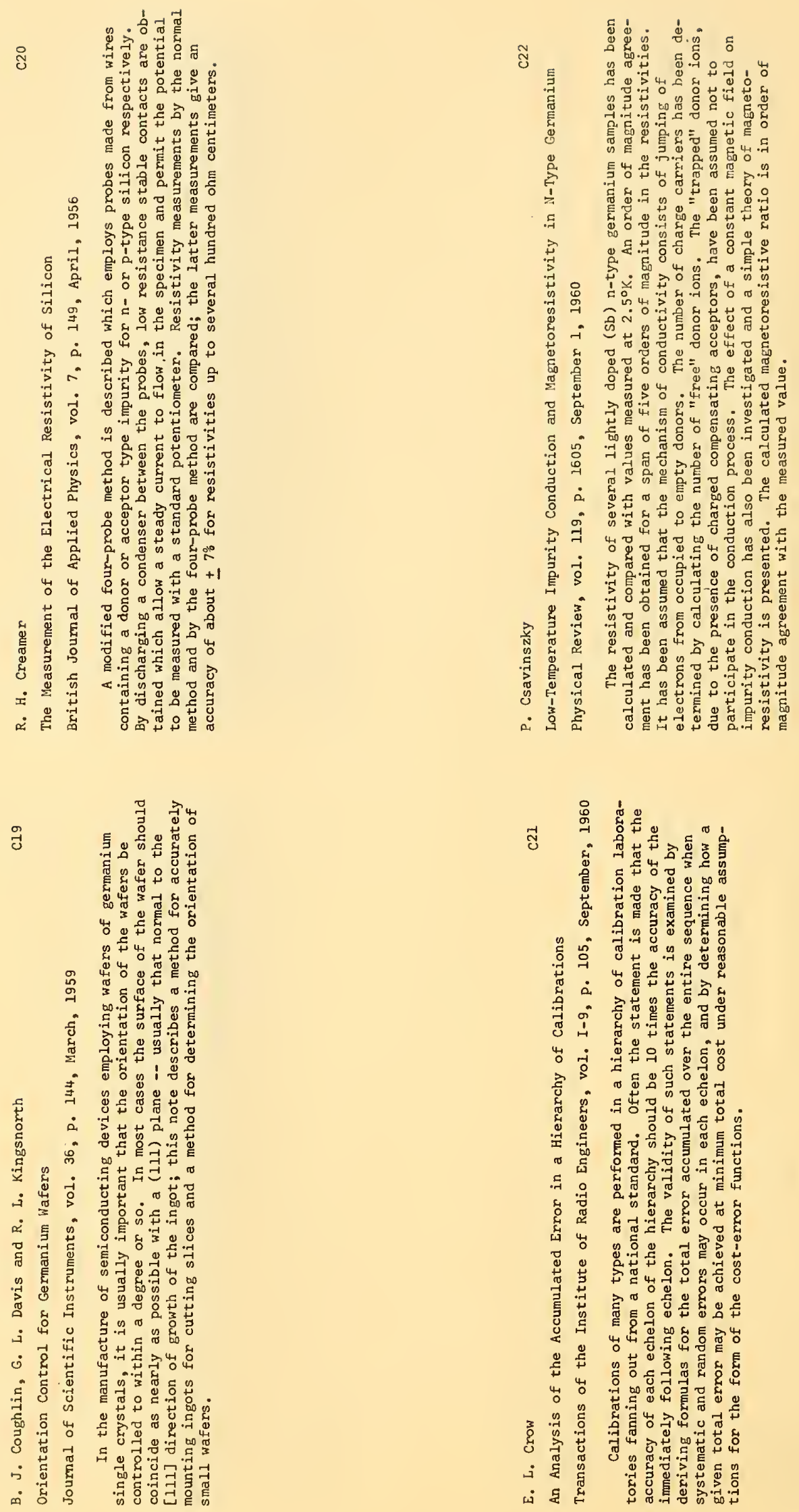
D.

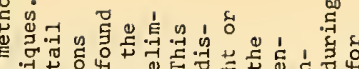

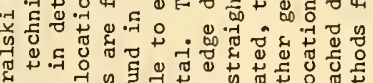

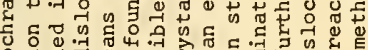

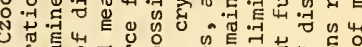

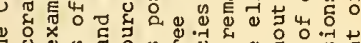

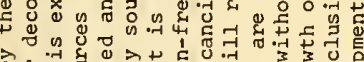

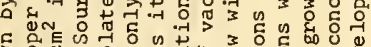

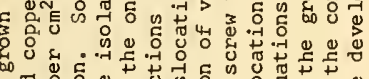

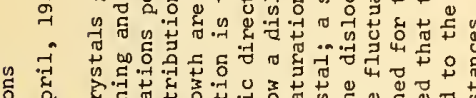

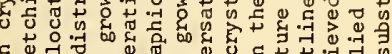
गु

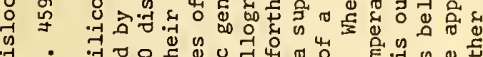

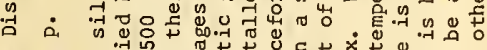

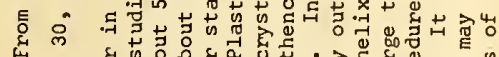
-i 氜 की 过

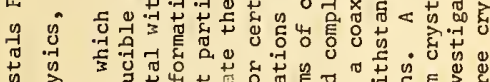

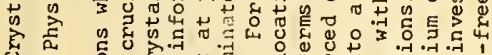

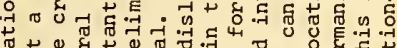

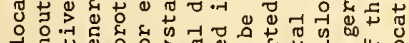

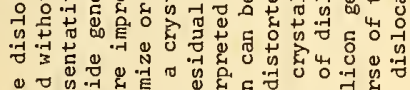

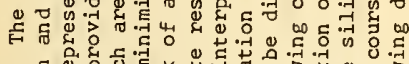

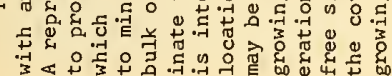

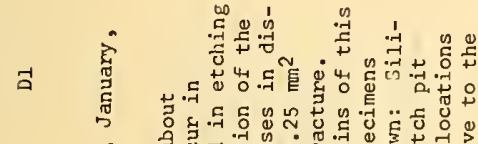

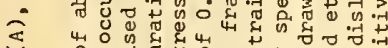

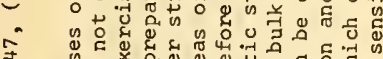

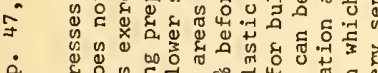

$\dot{a} \quad 4 \quad 0.7$.

$\neq$ ₹

तै

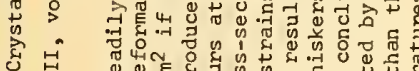

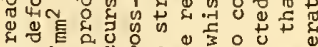

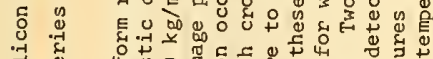

-7

क व

品

出

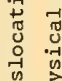

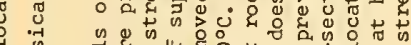

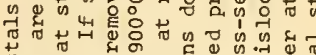

落

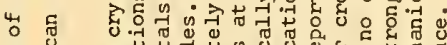

o

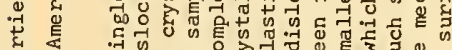

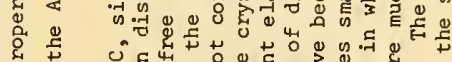

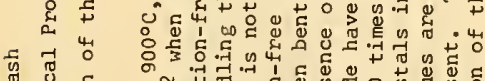

婴

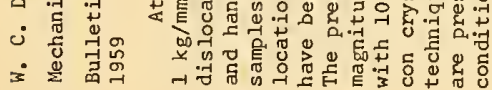

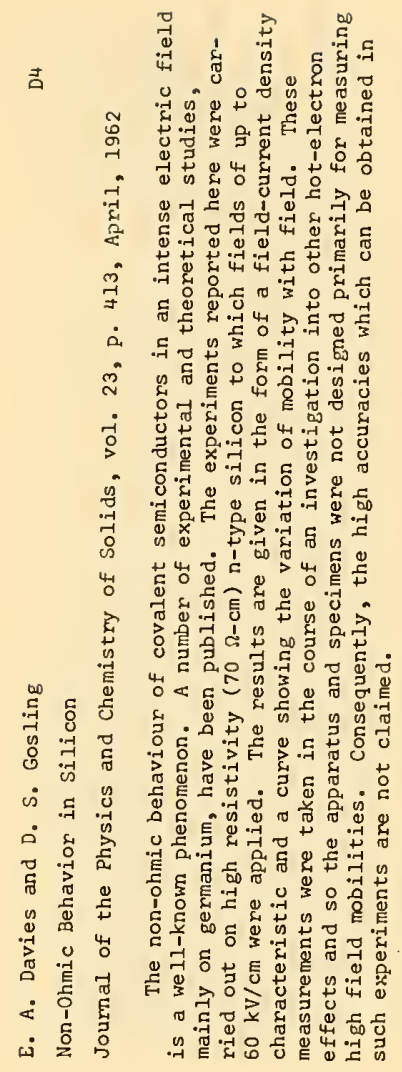

ฉ

品

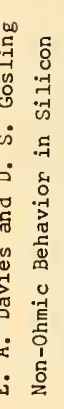

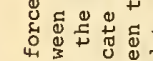

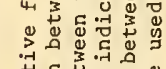

-

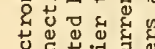

-

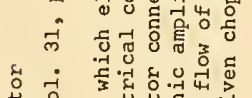

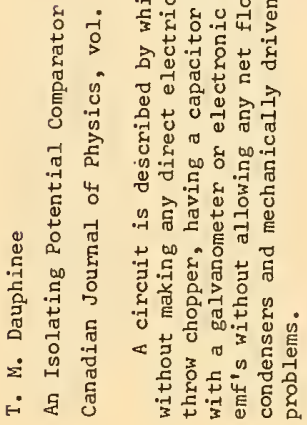

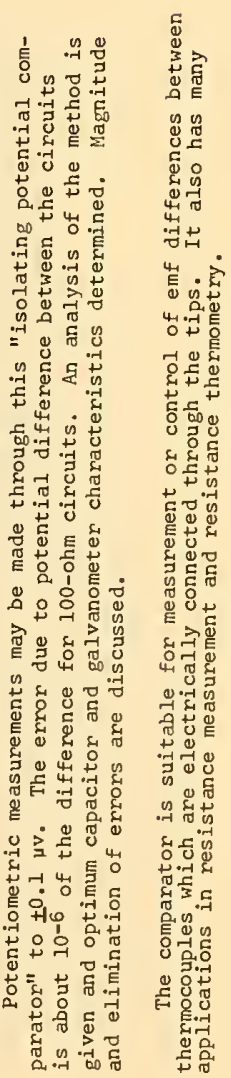




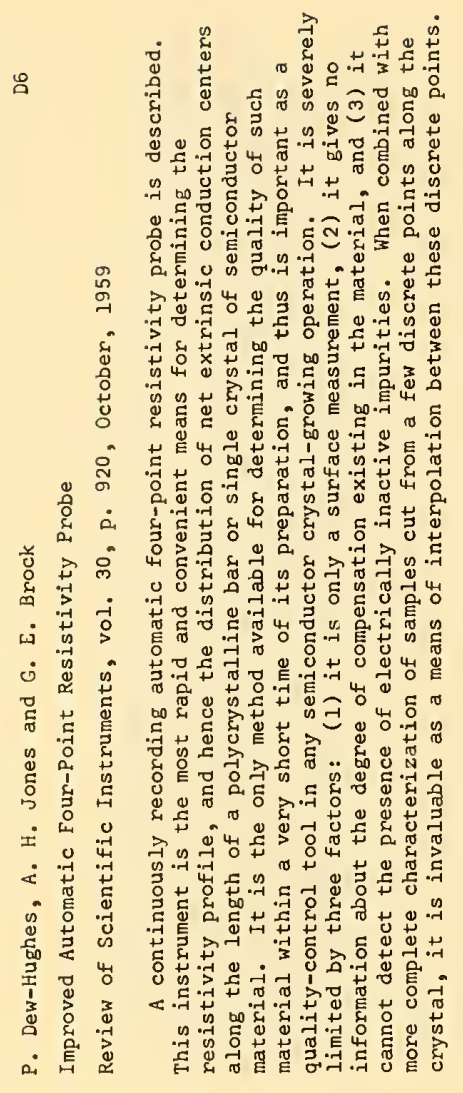

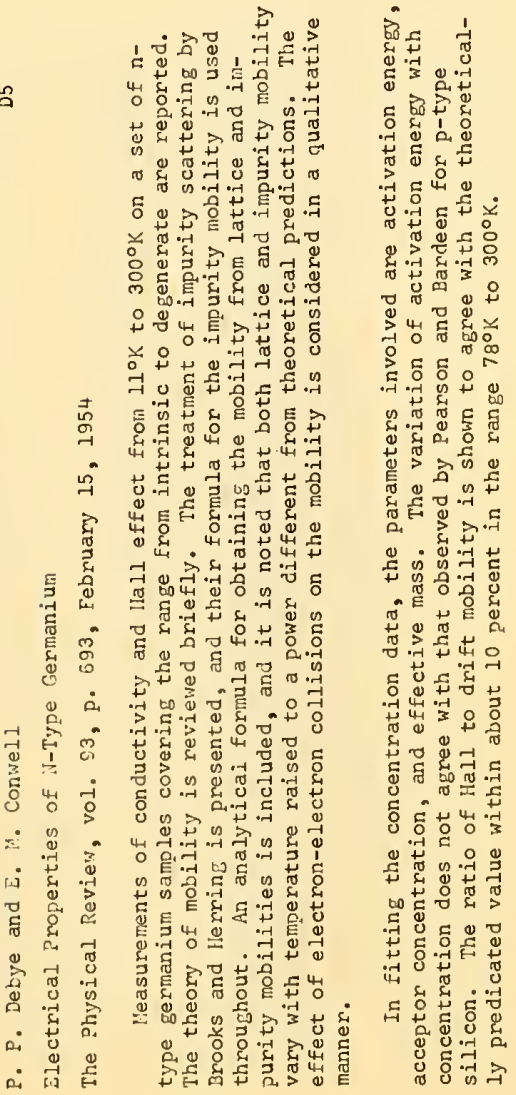

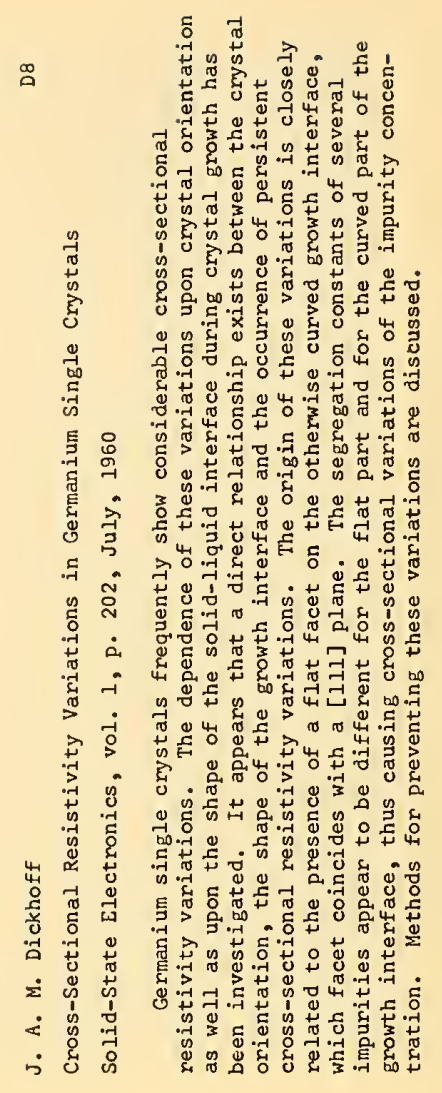



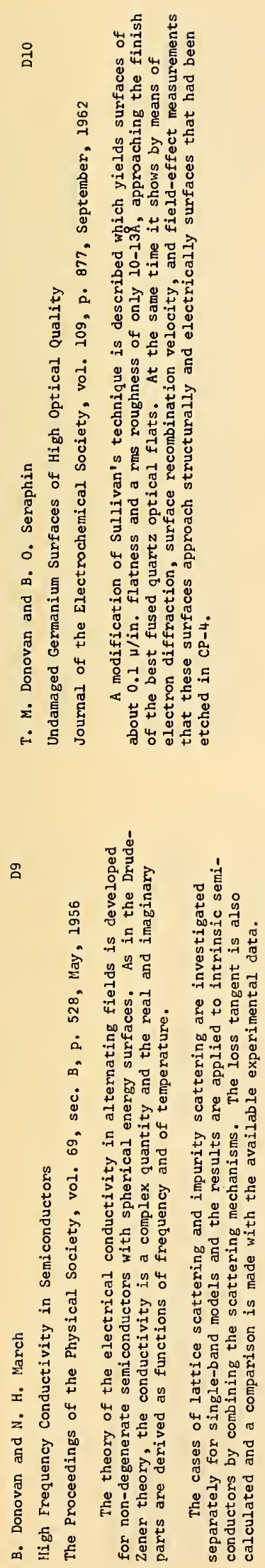
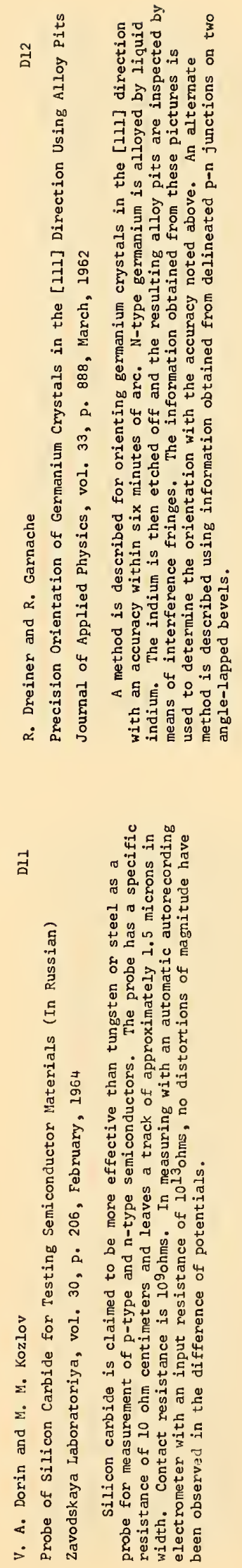

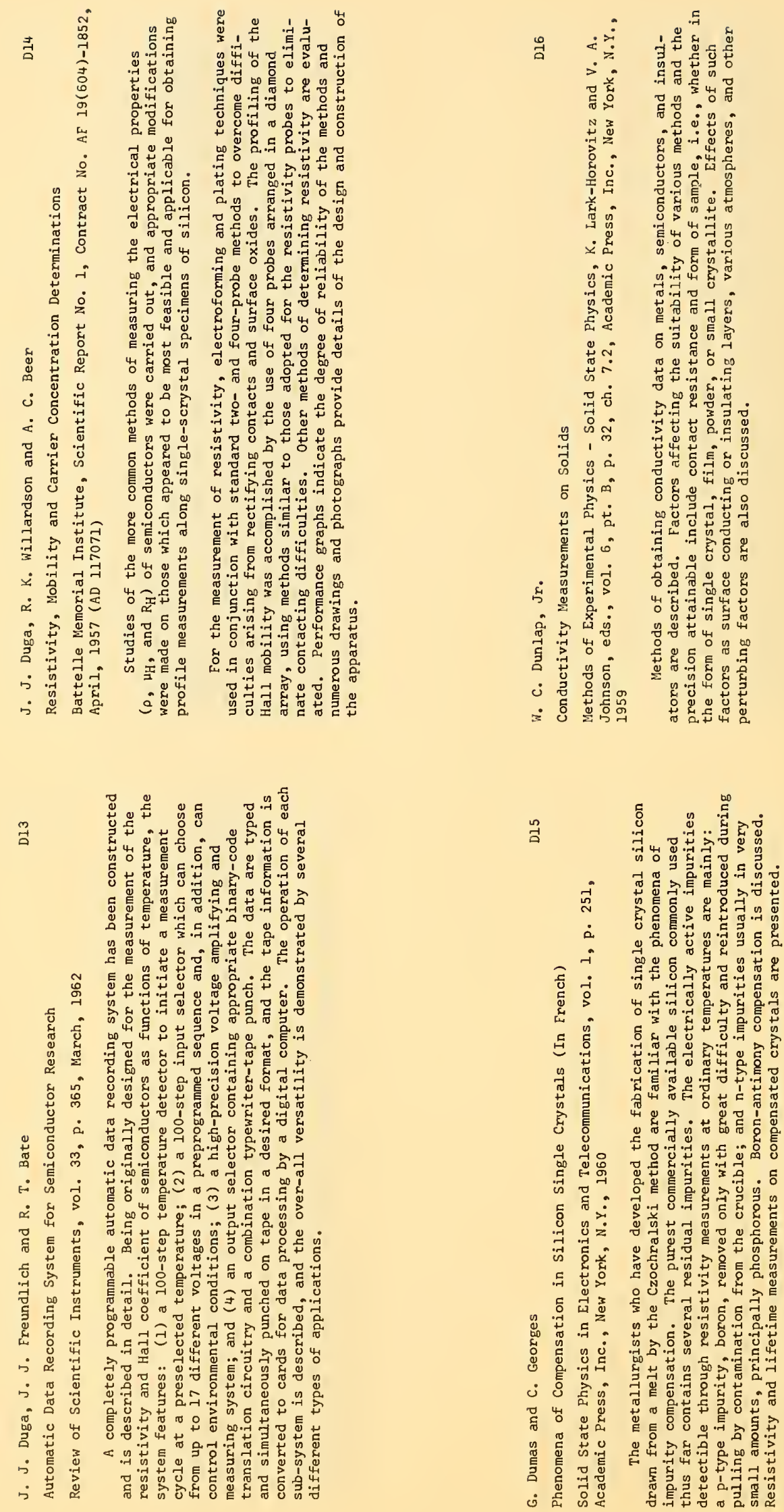

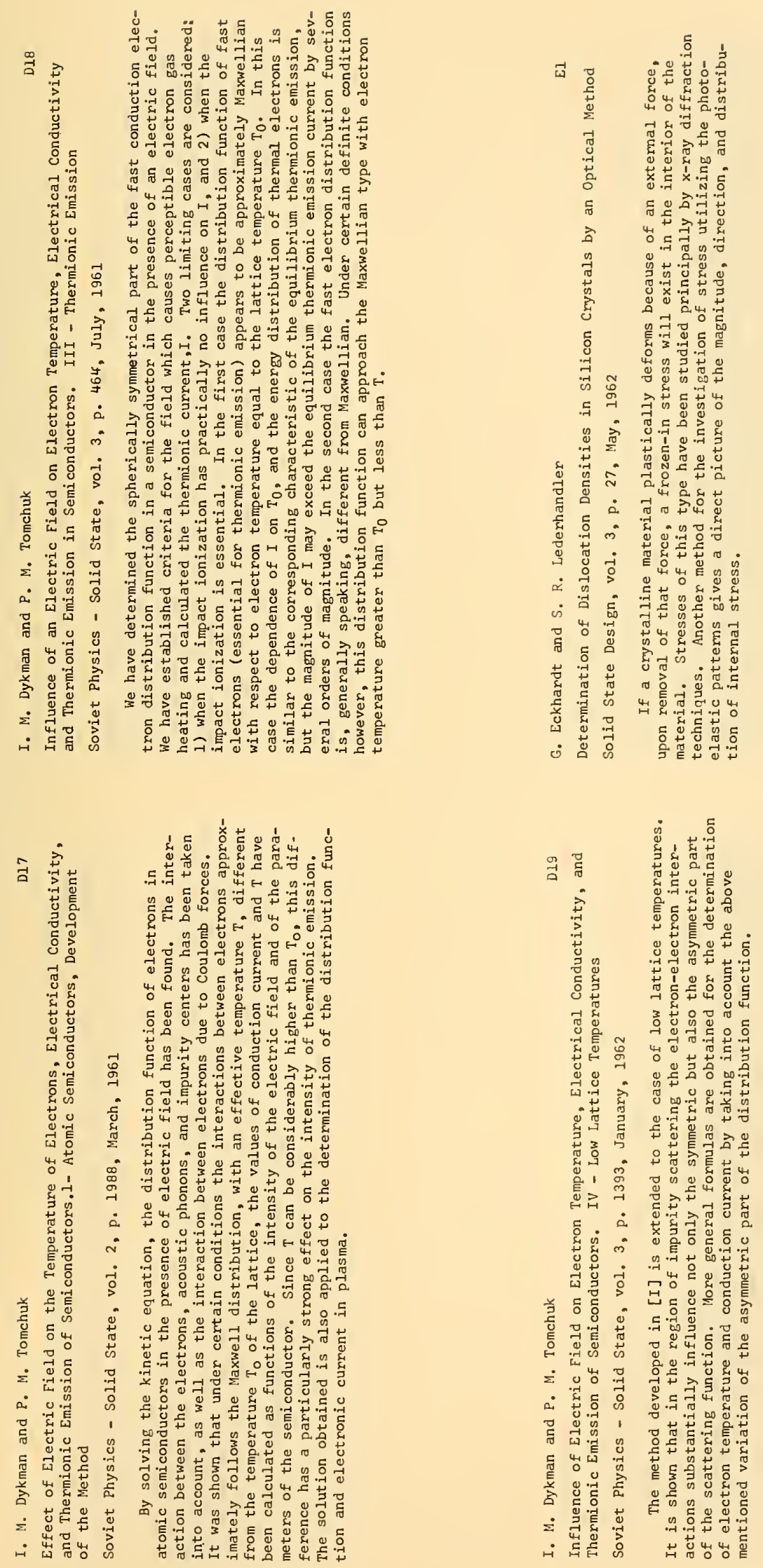

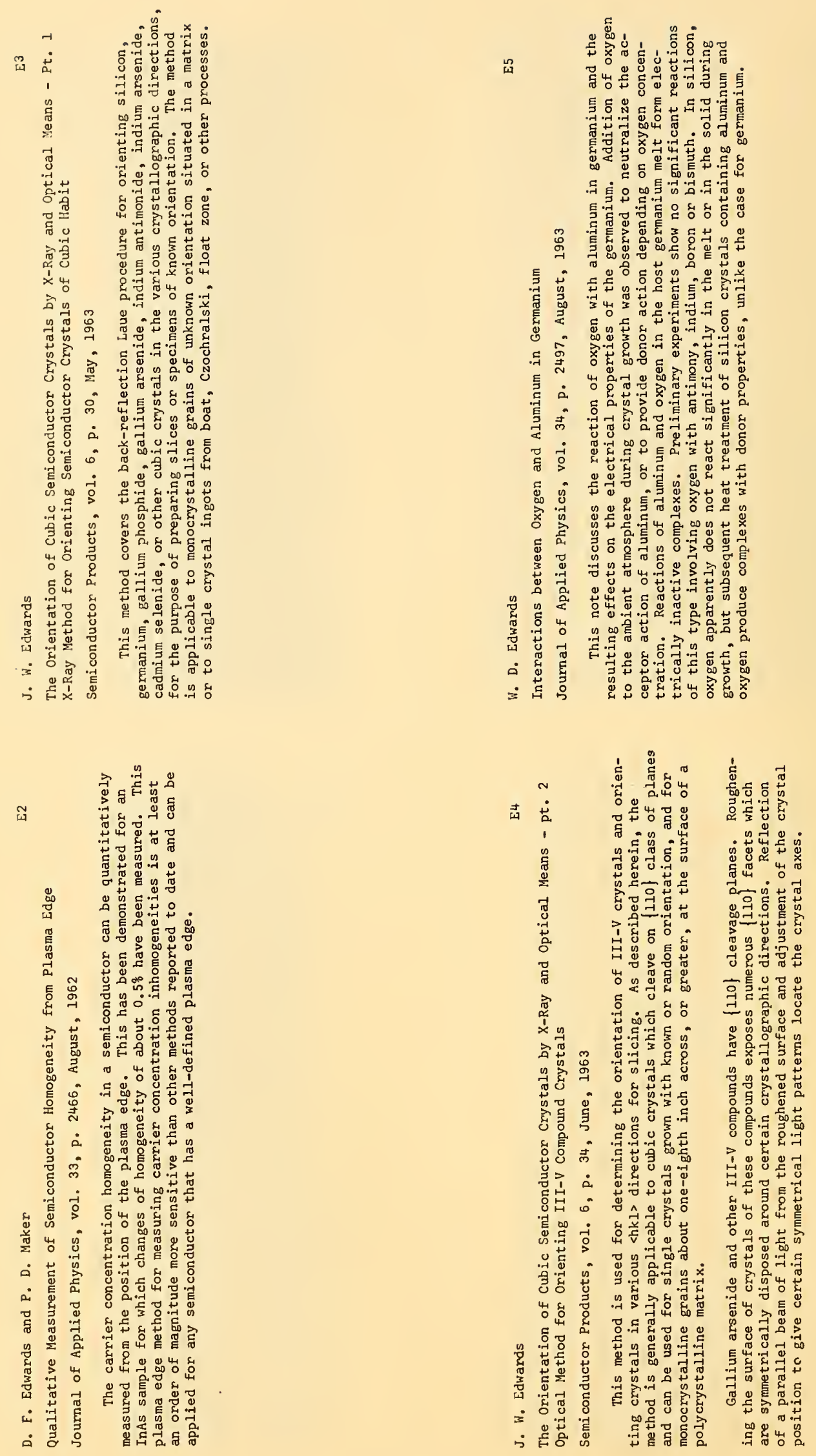

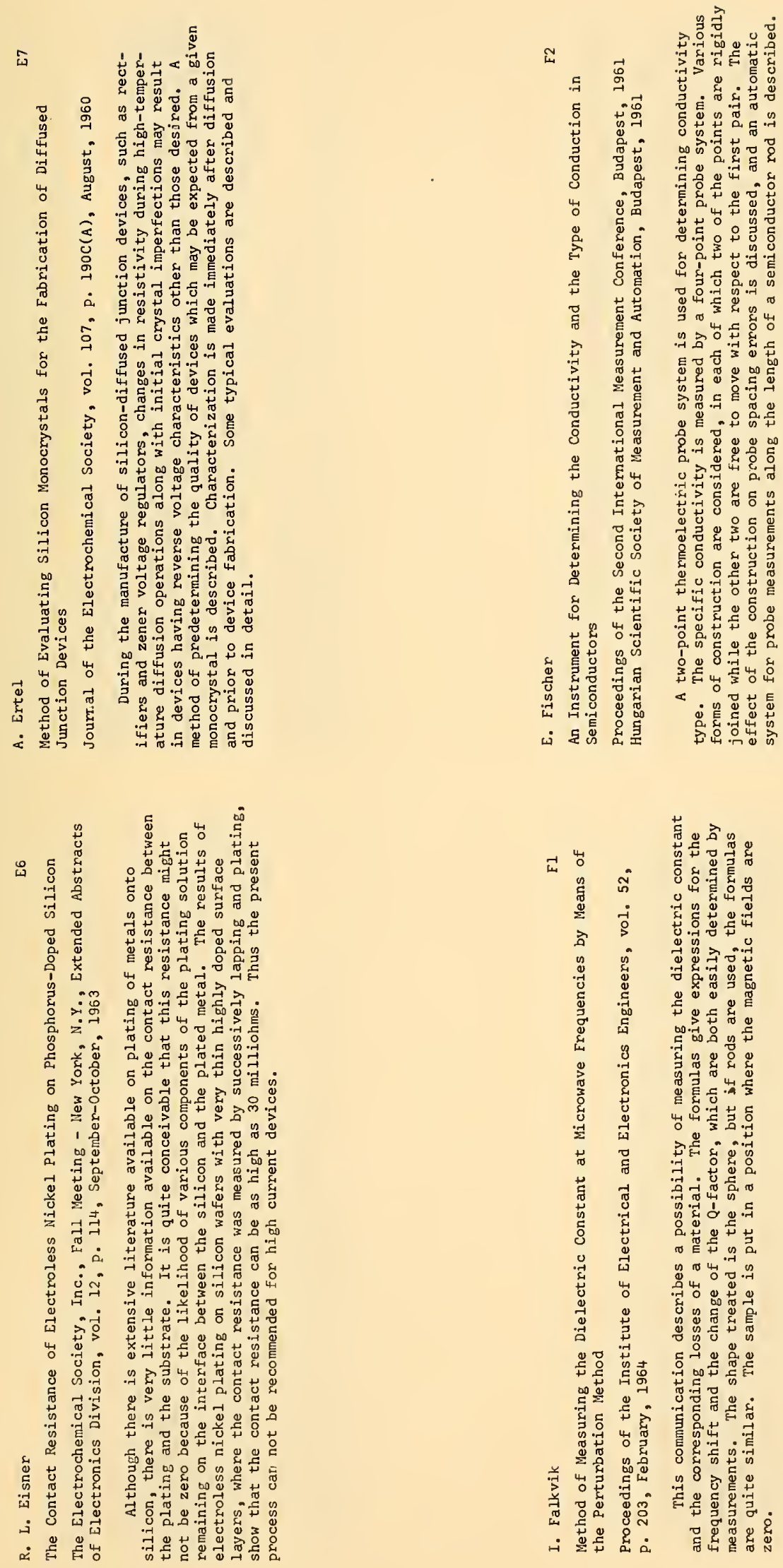

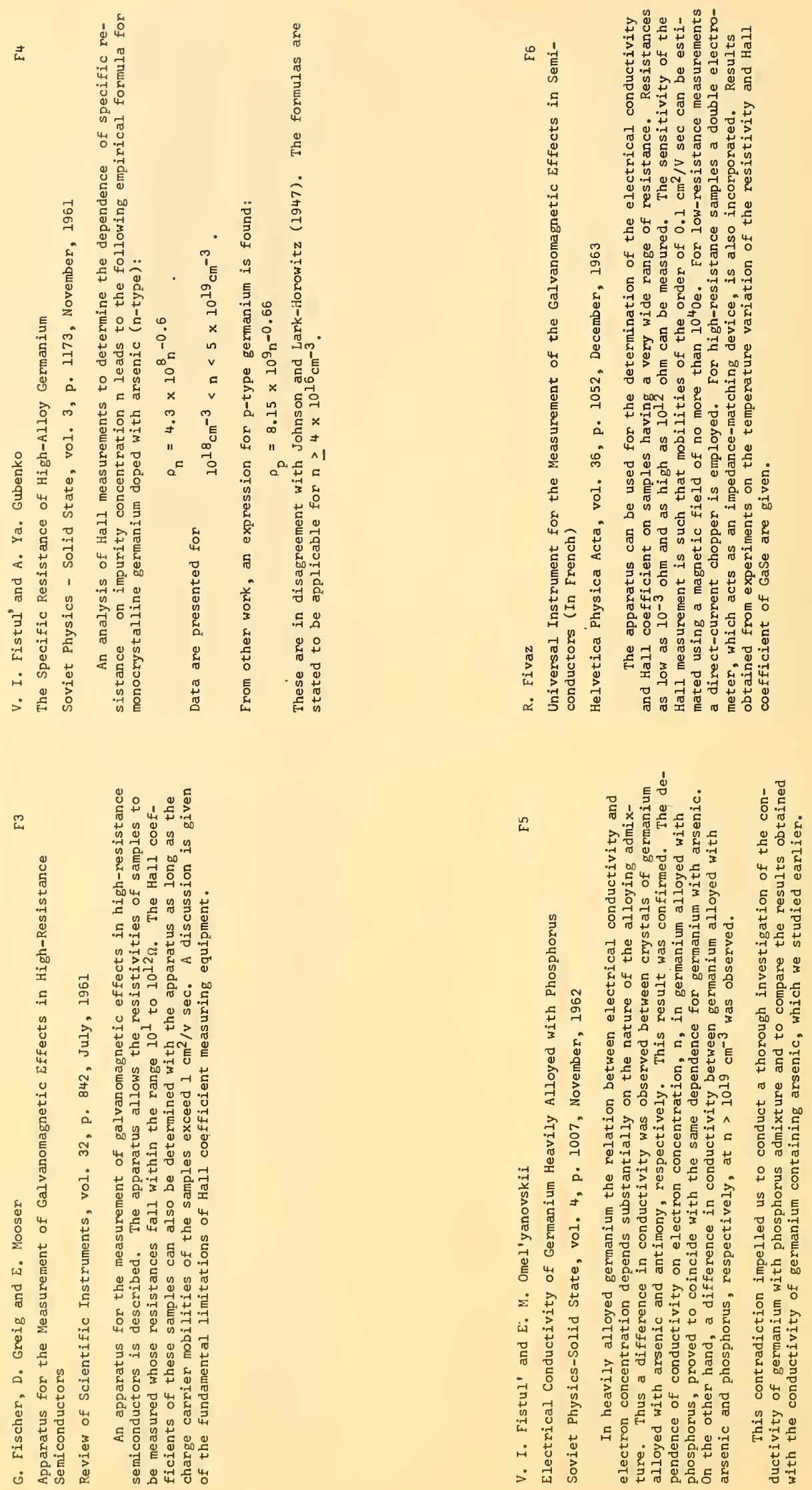

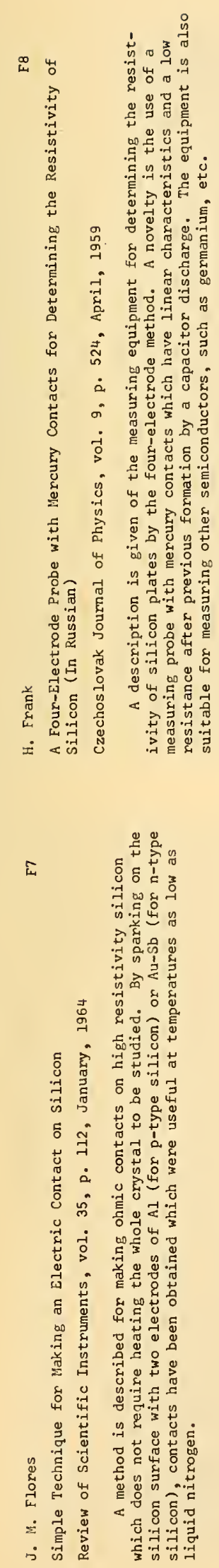
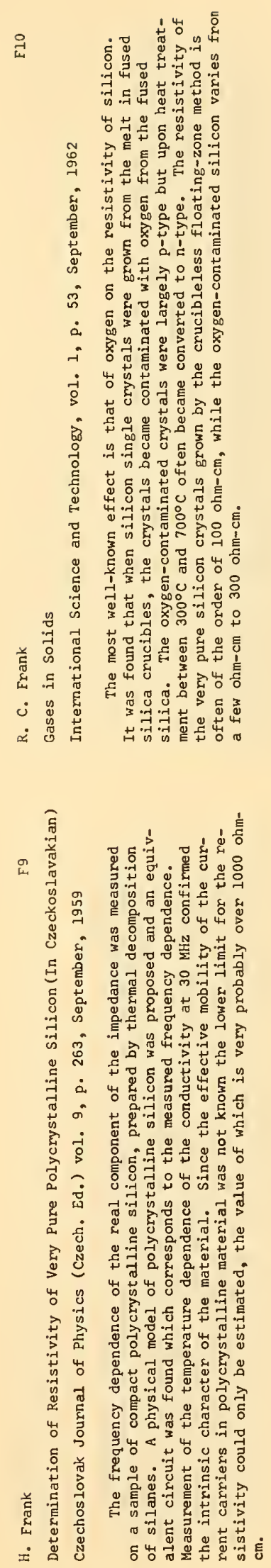

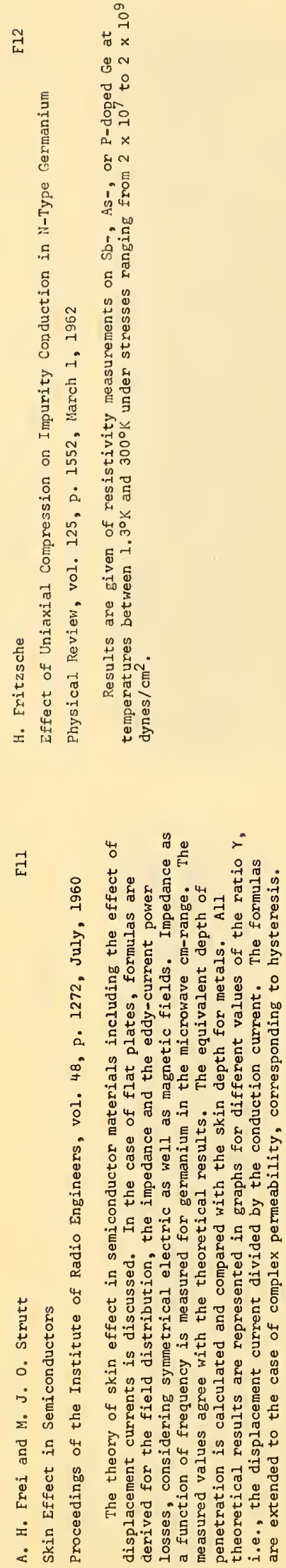

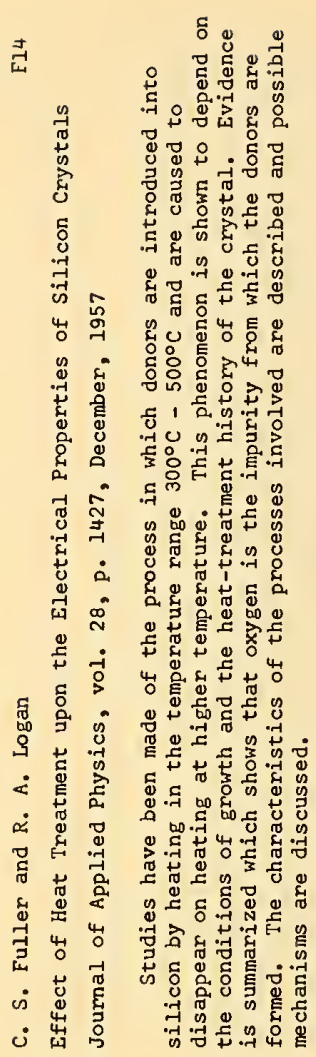

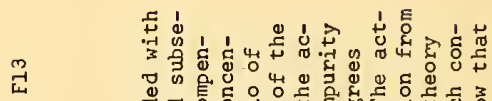

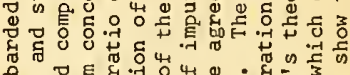

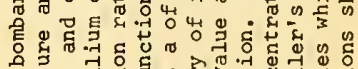

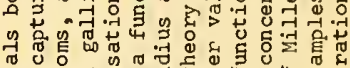

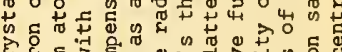

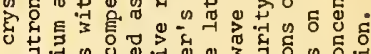

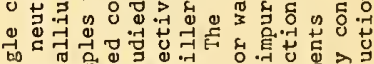

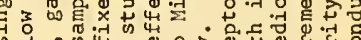

⿻日木

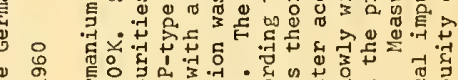

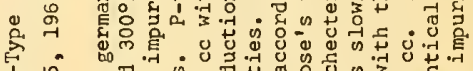

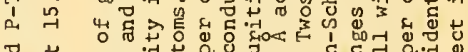

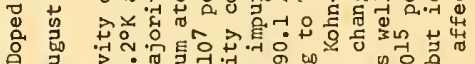

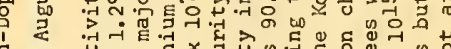

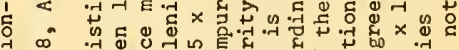

学

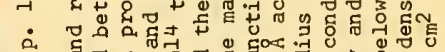

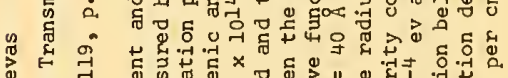

势 ने

- न

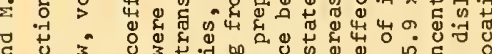

ช

\&

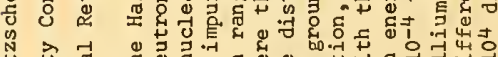

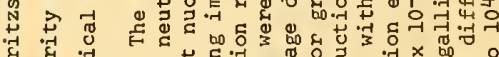

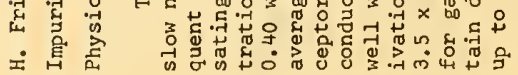




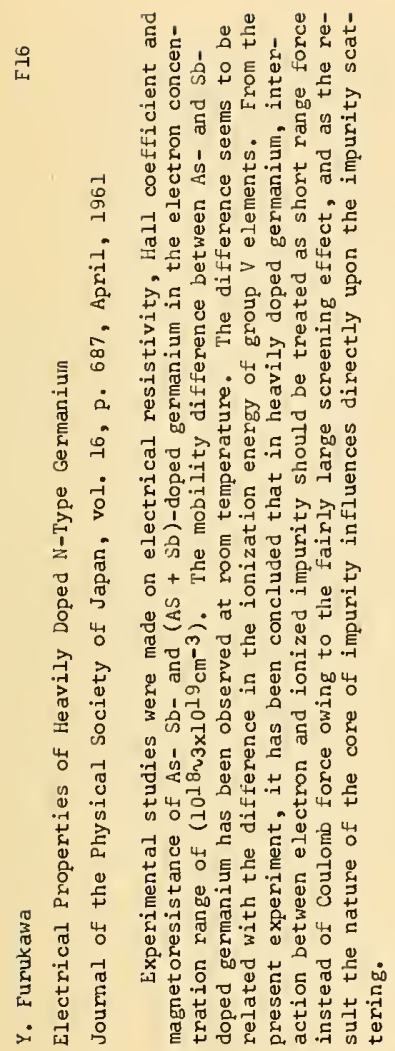

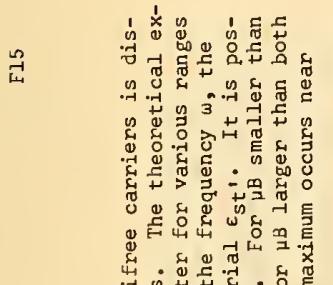

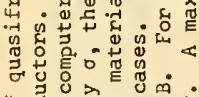

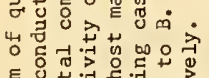

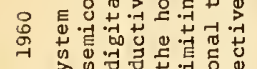

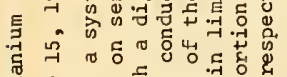

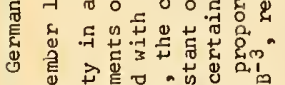

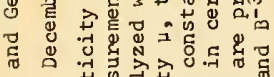

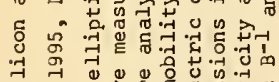

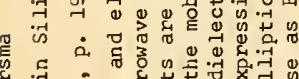

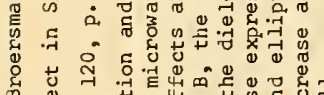

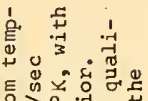

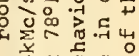

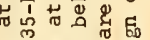

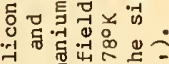

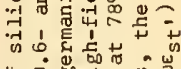

出 $\sigma^{\circ}$ 政

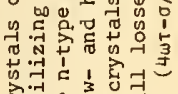

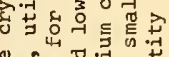

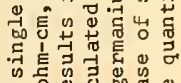

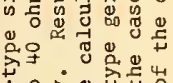

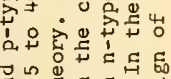

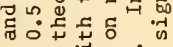

1 E 둔

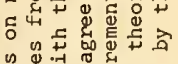

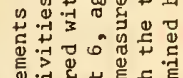

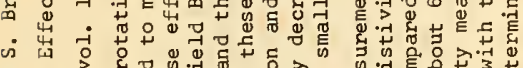

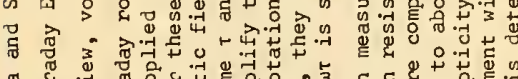

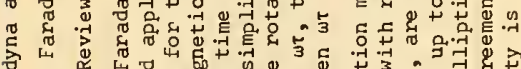

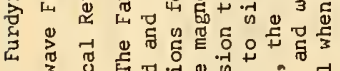

क

$\dot{x}$ 年

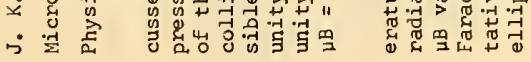
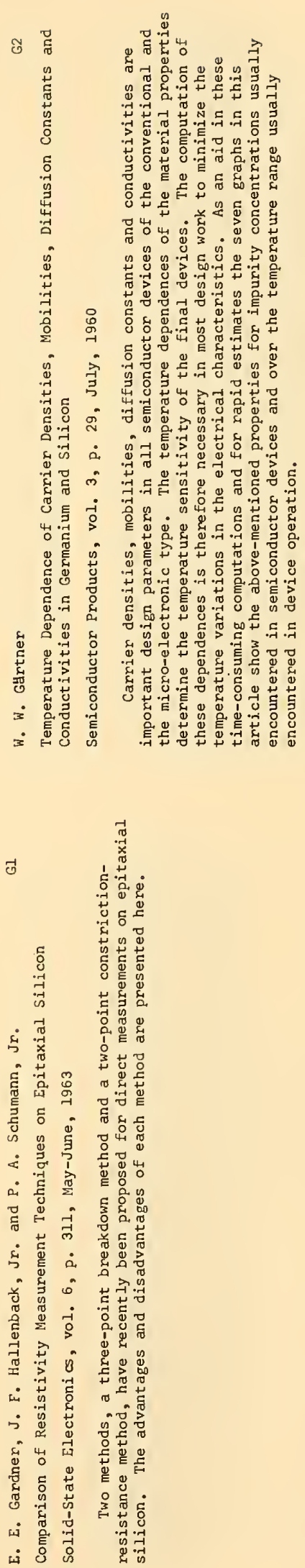

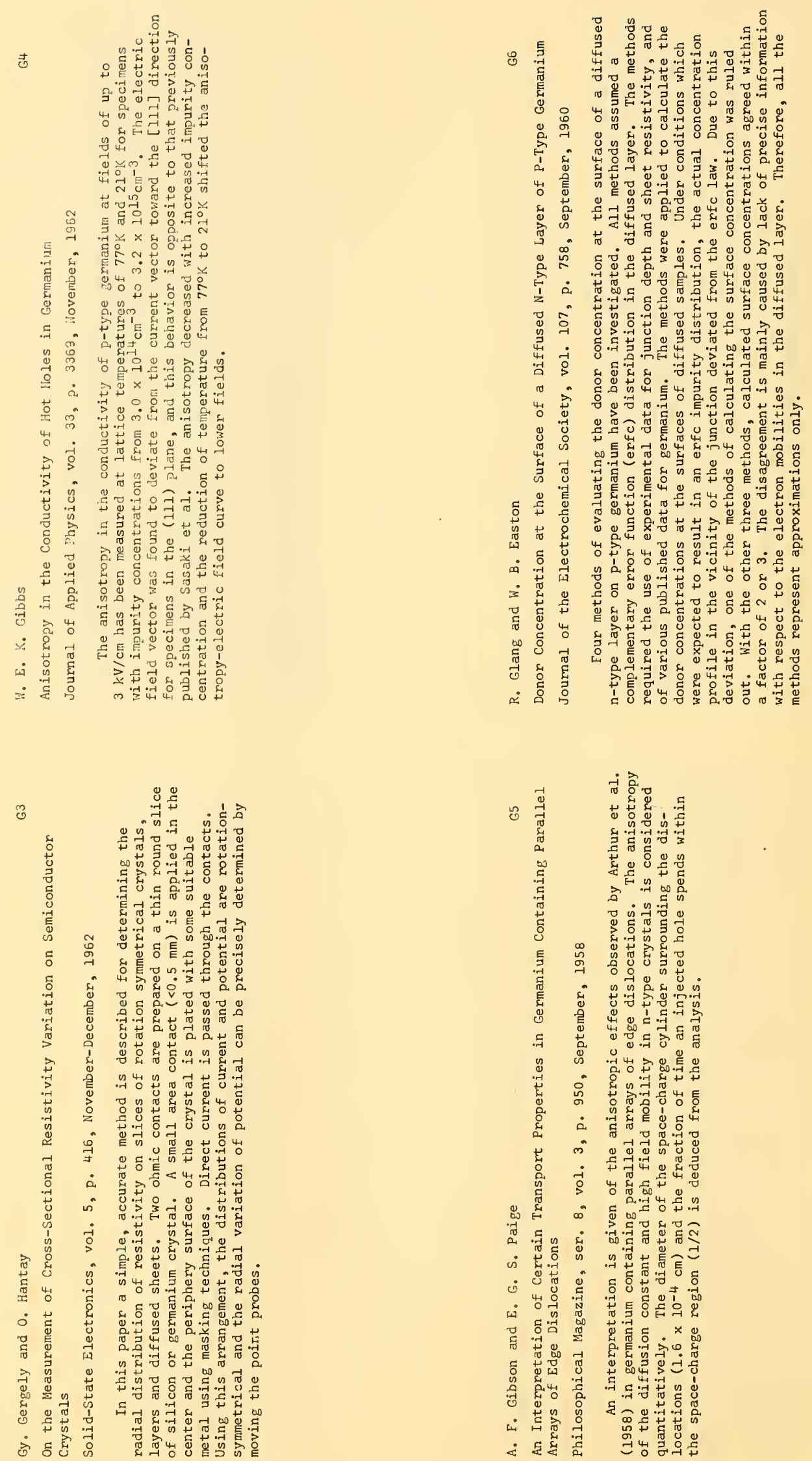

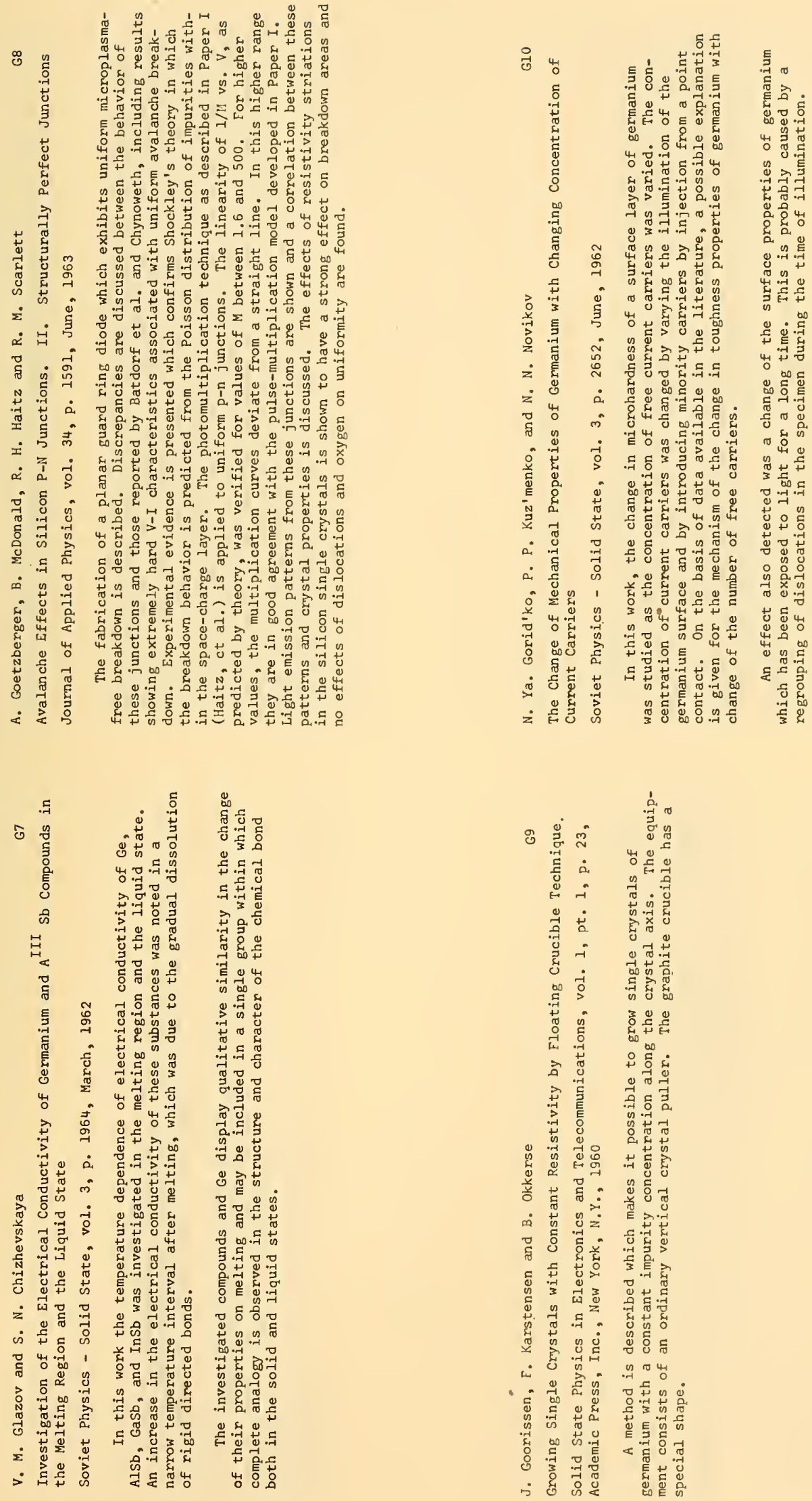

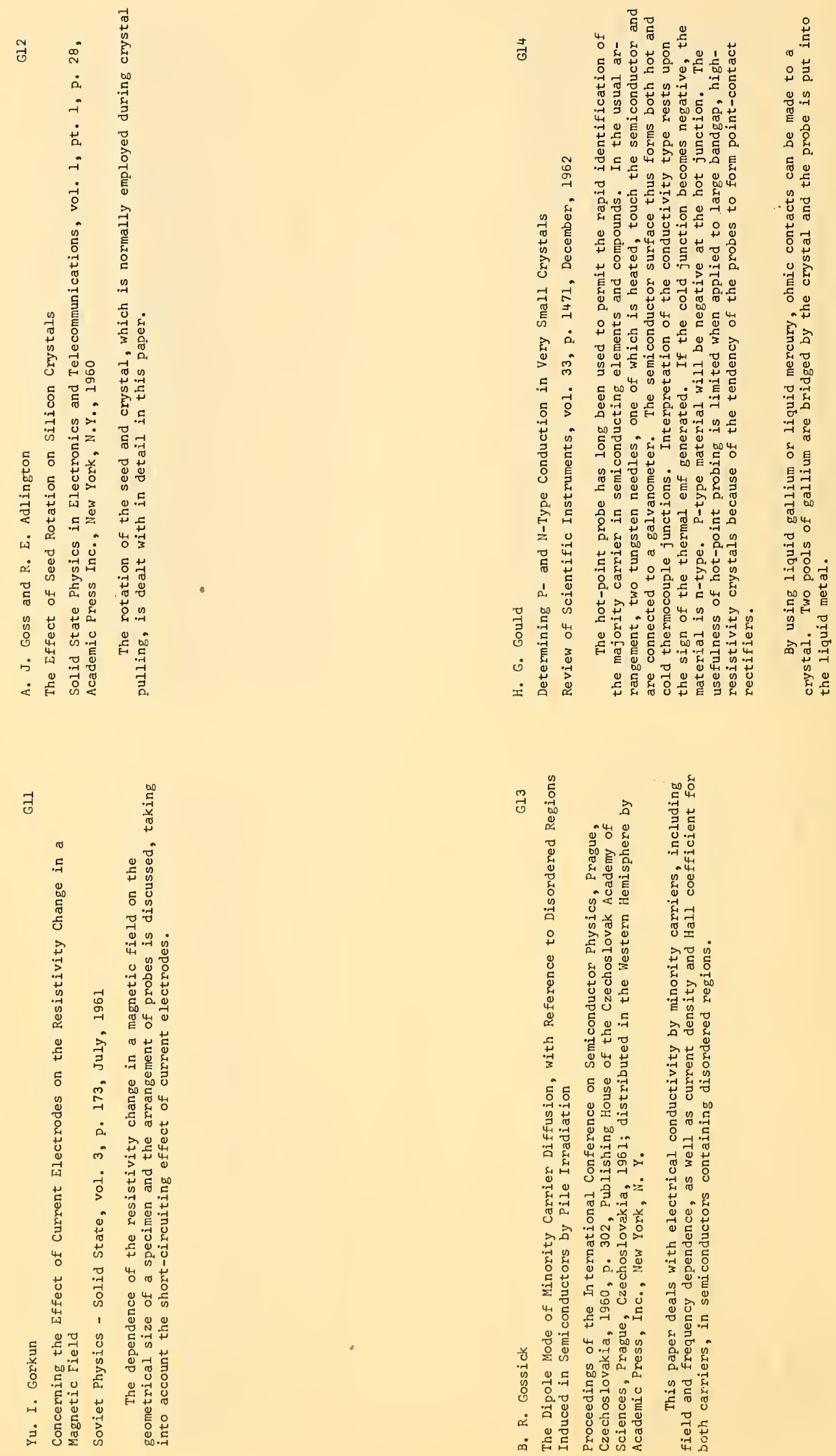

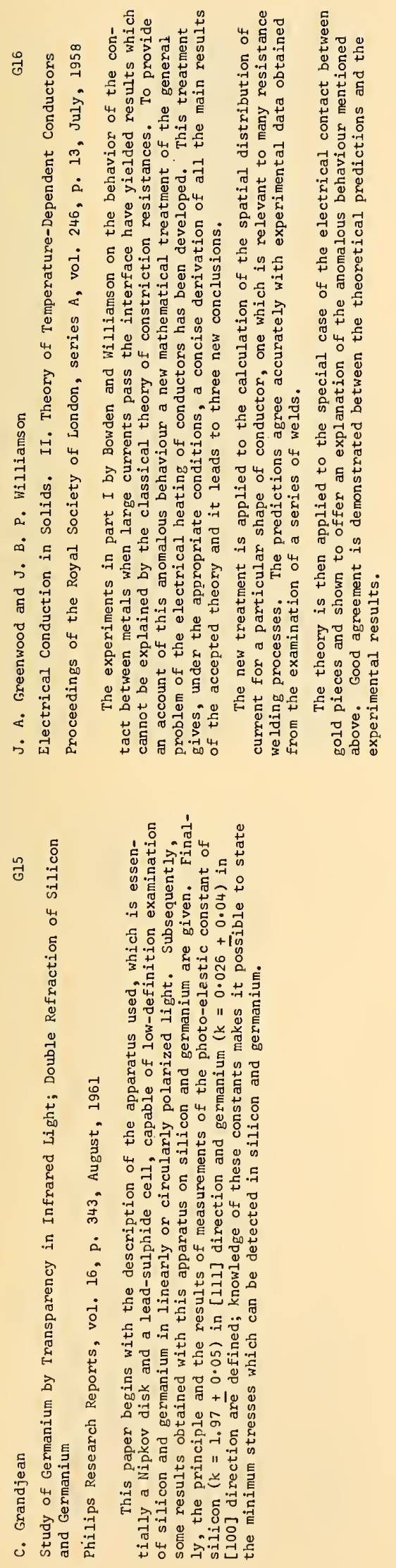
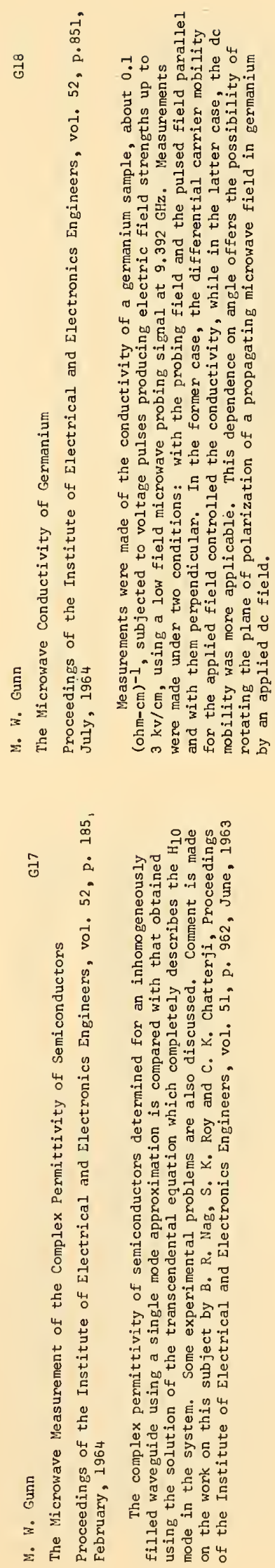

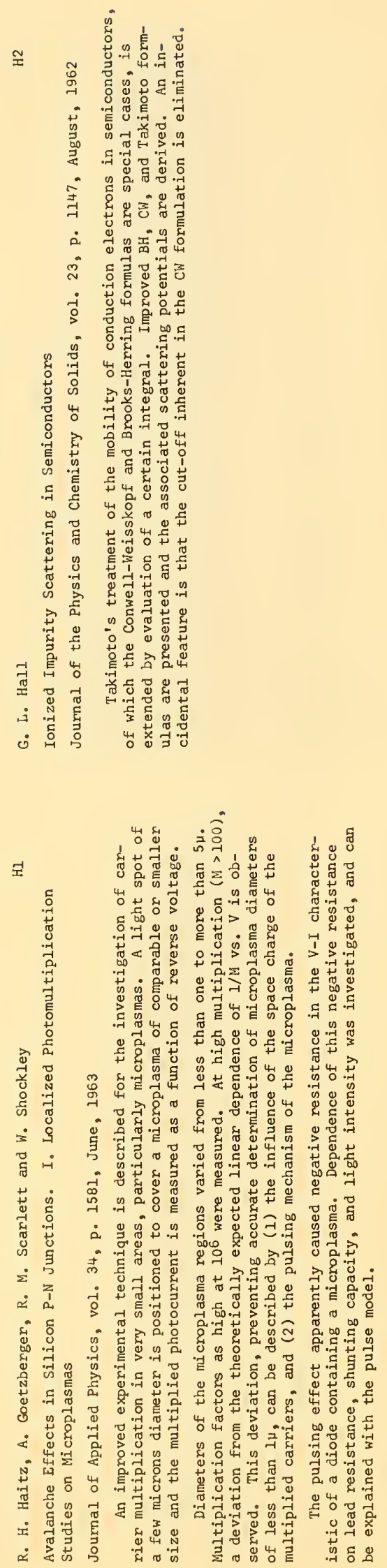
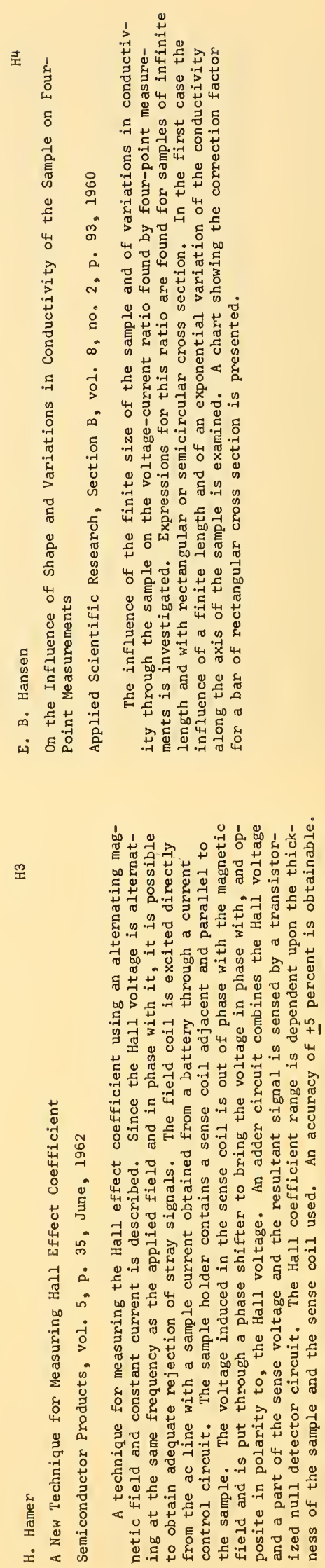


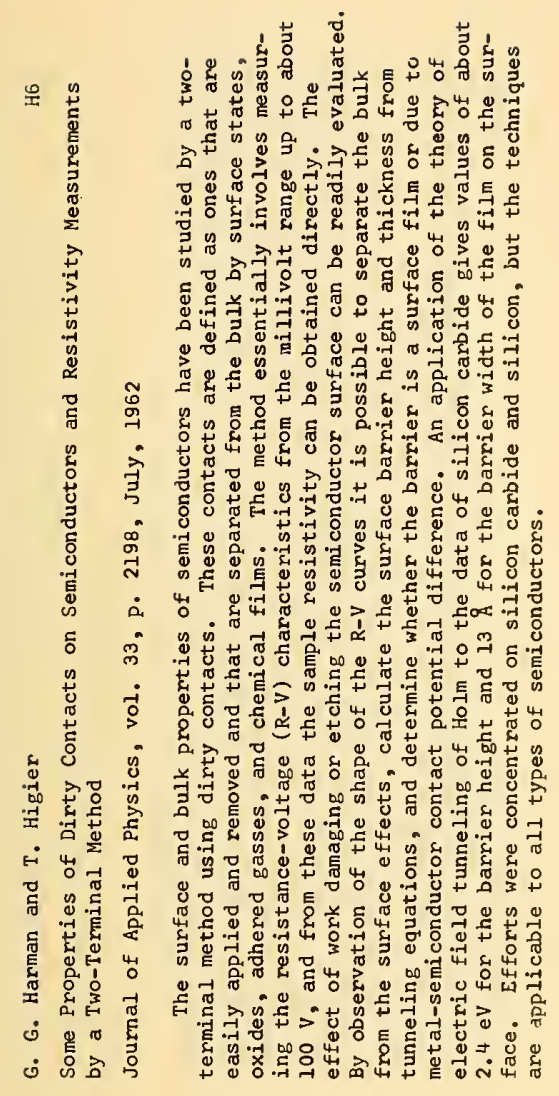

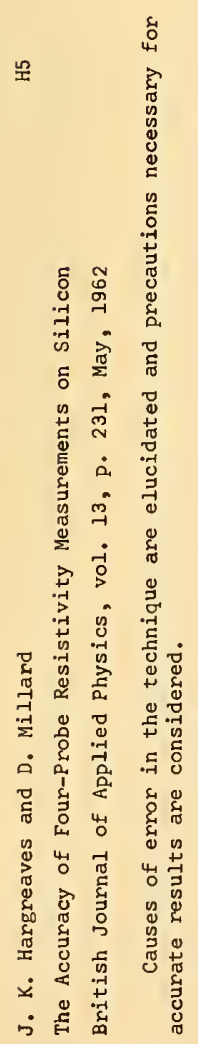
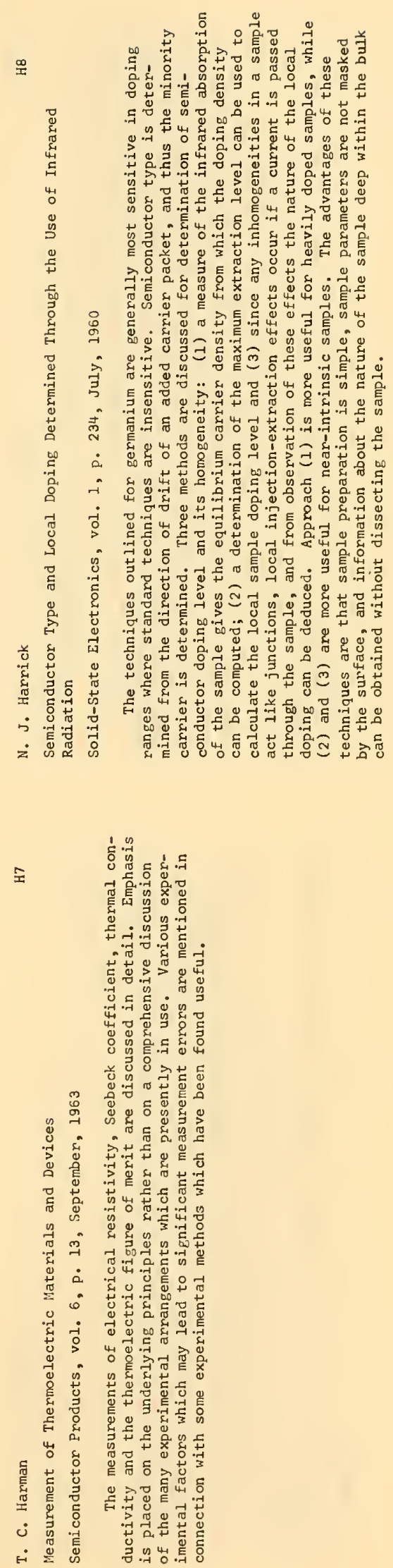

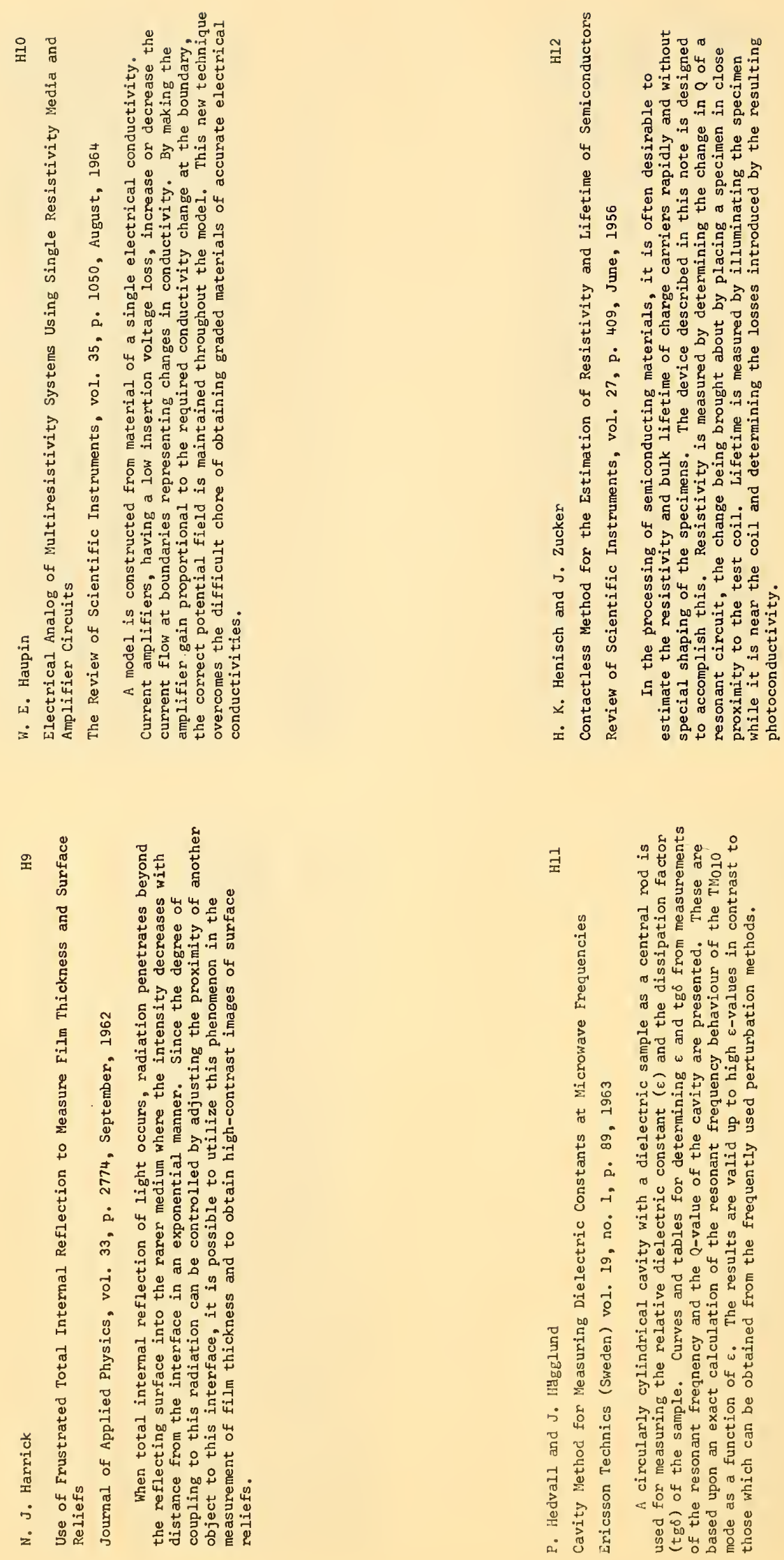

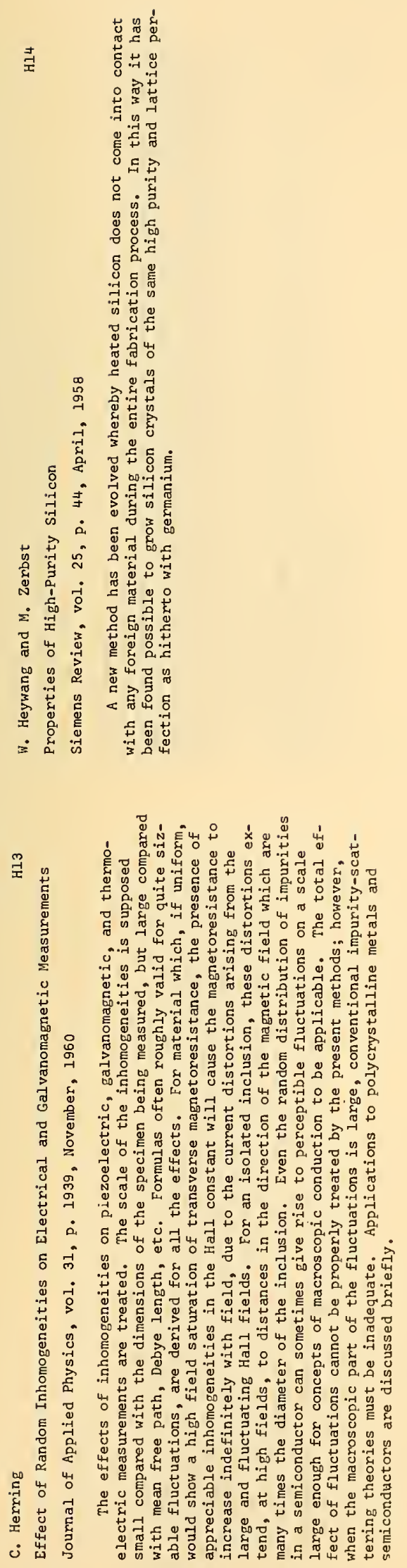
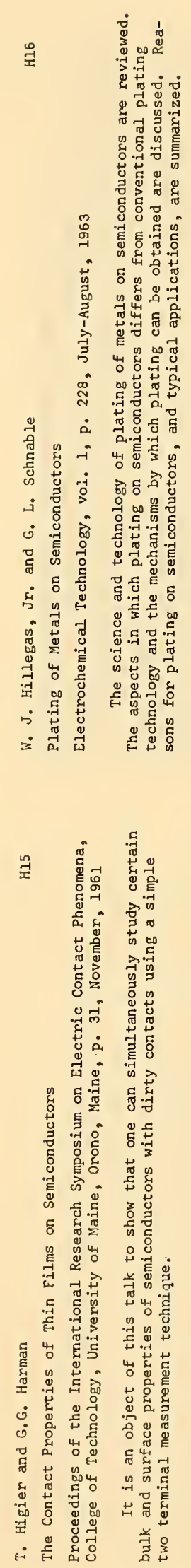

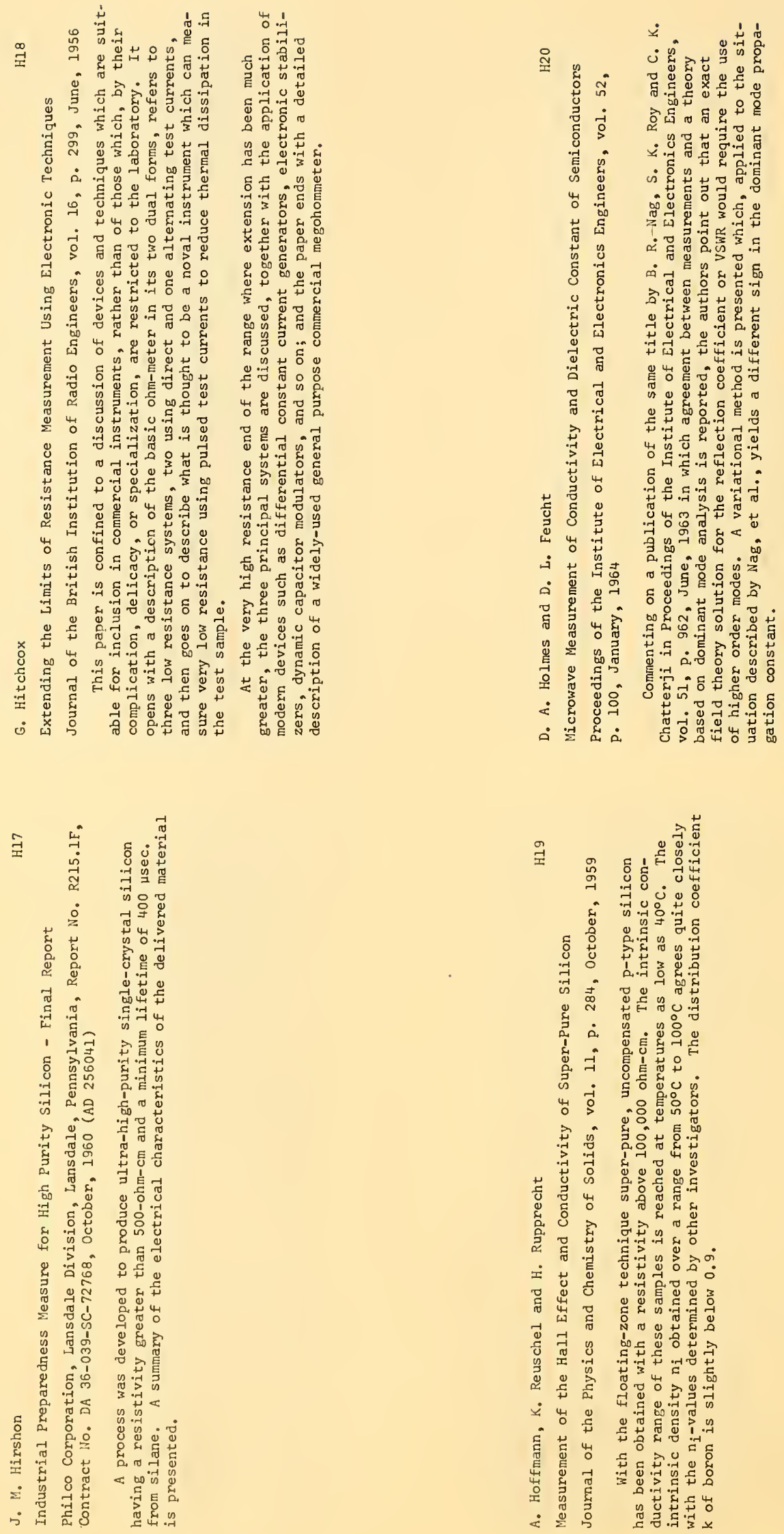


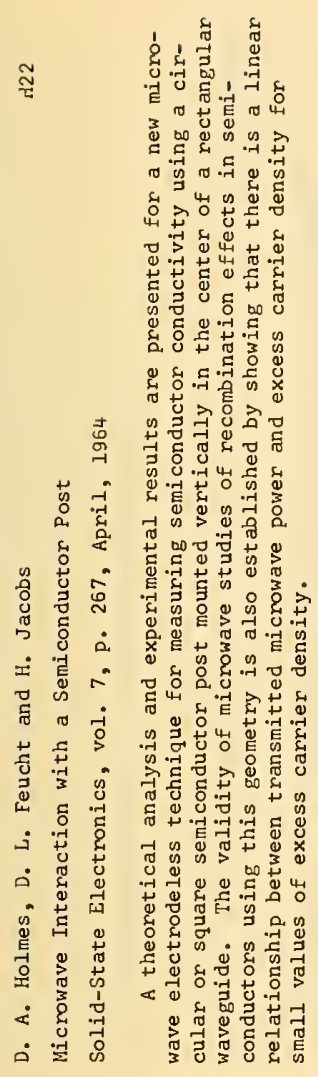

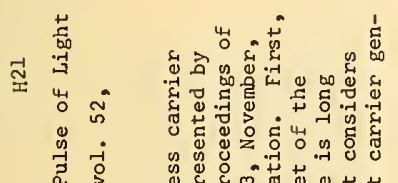

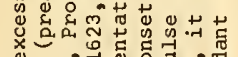

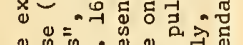

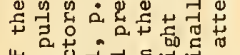

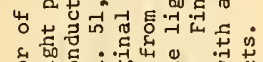

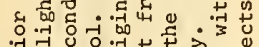

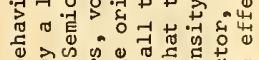

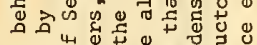

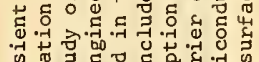

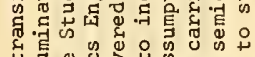

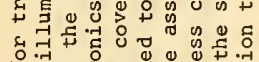

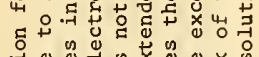

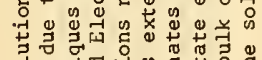

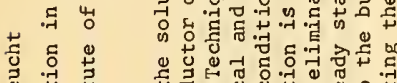

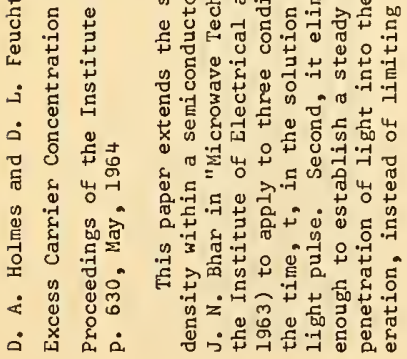
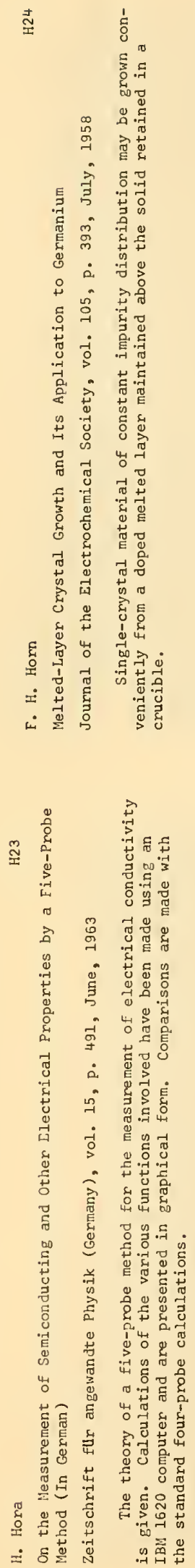

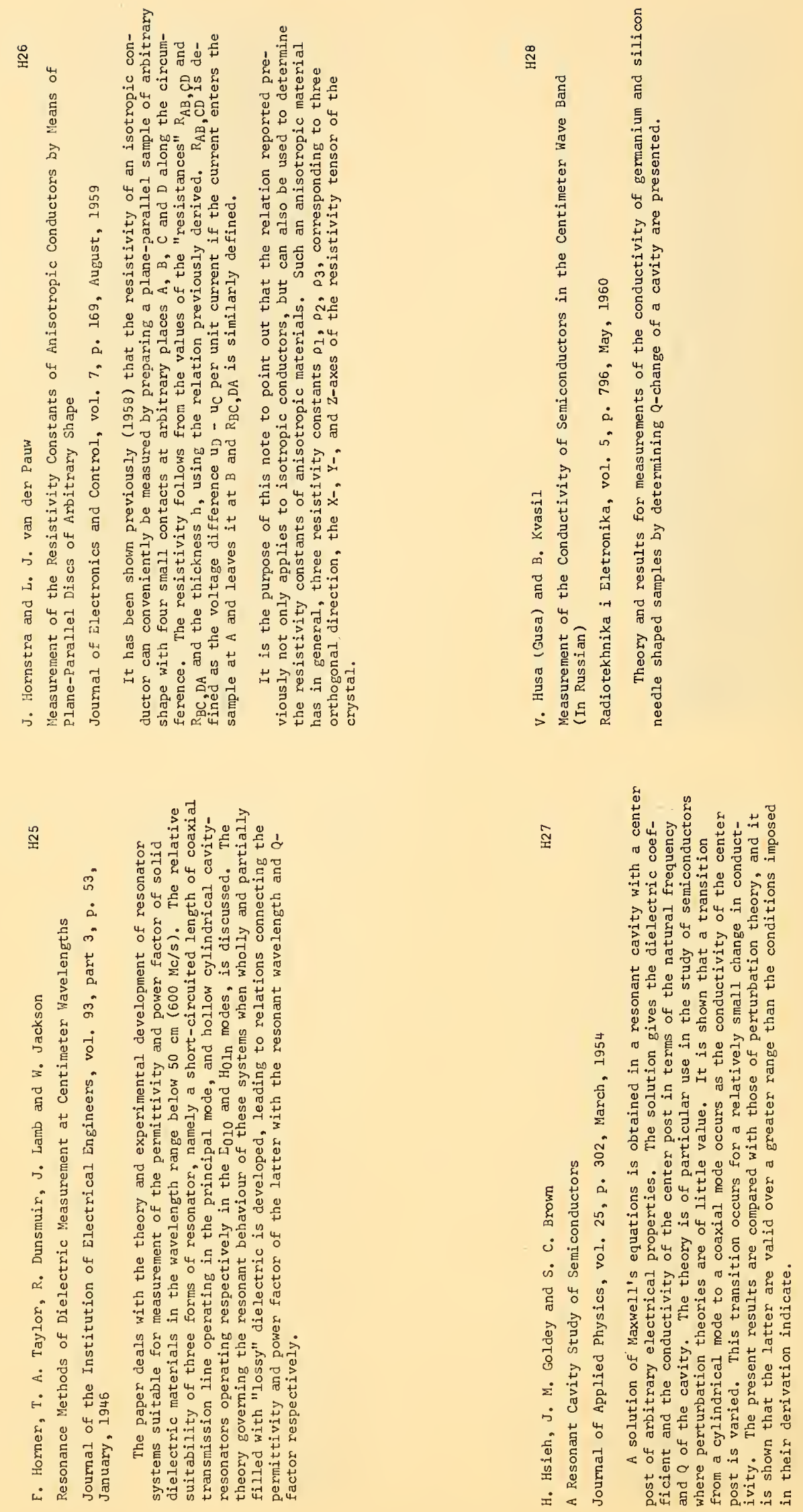

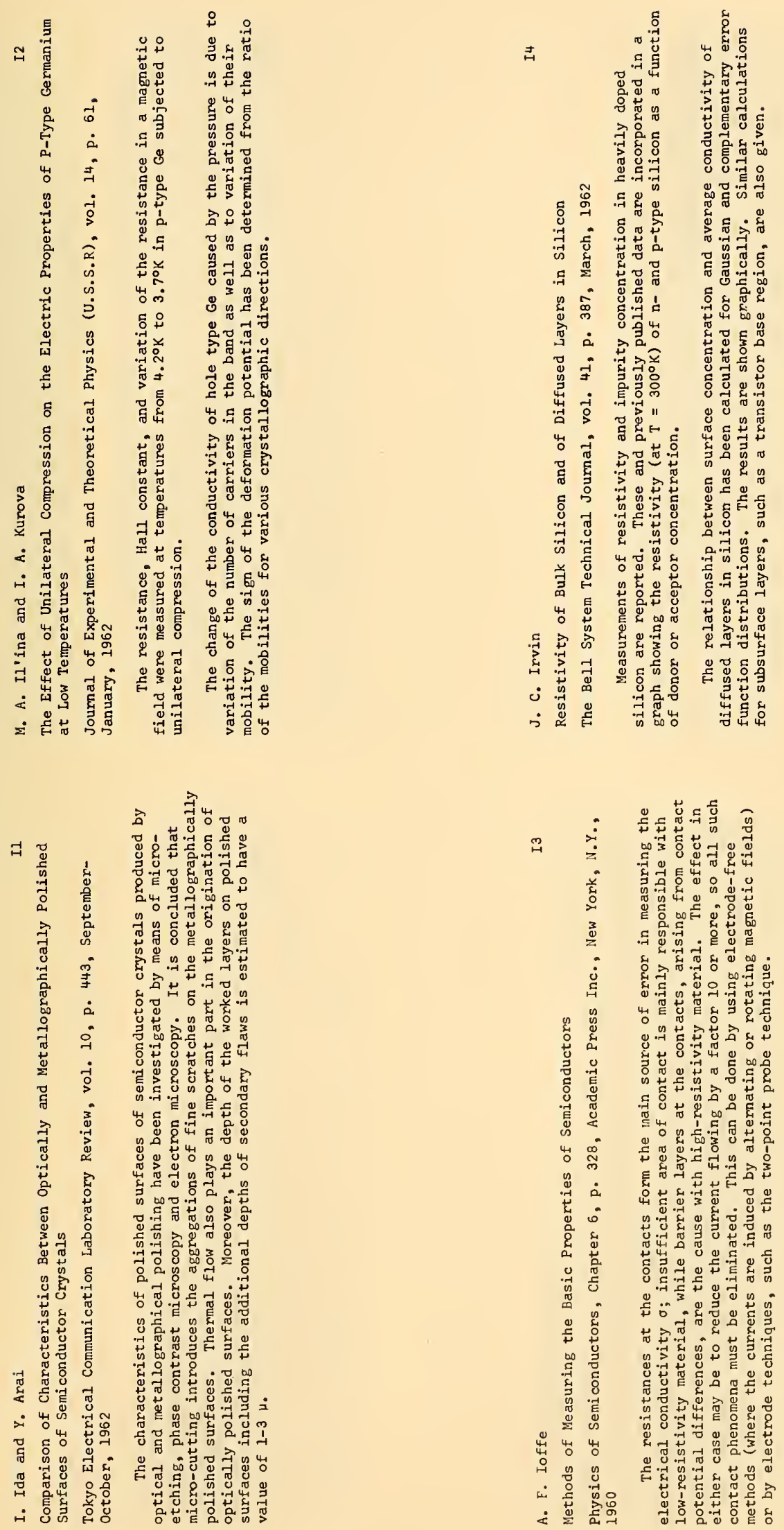

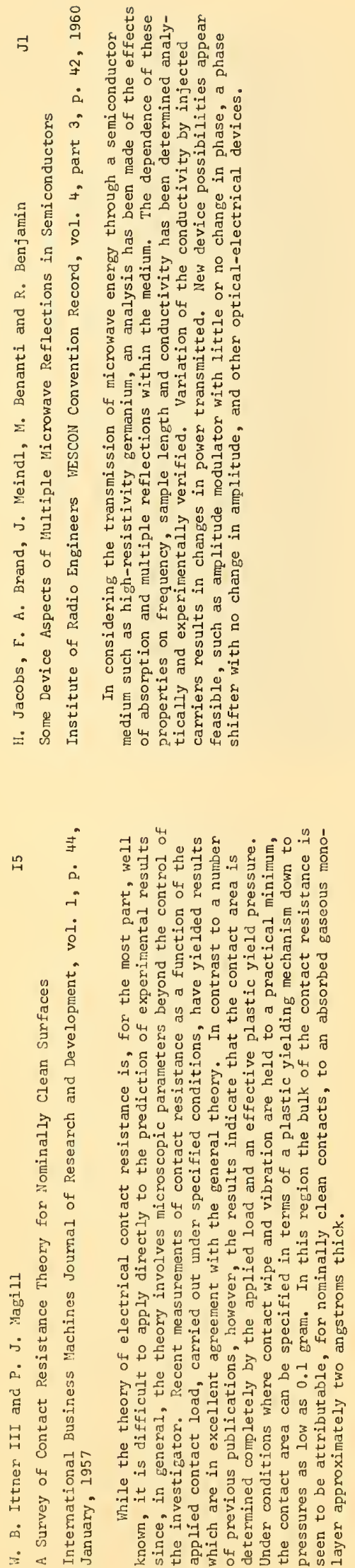
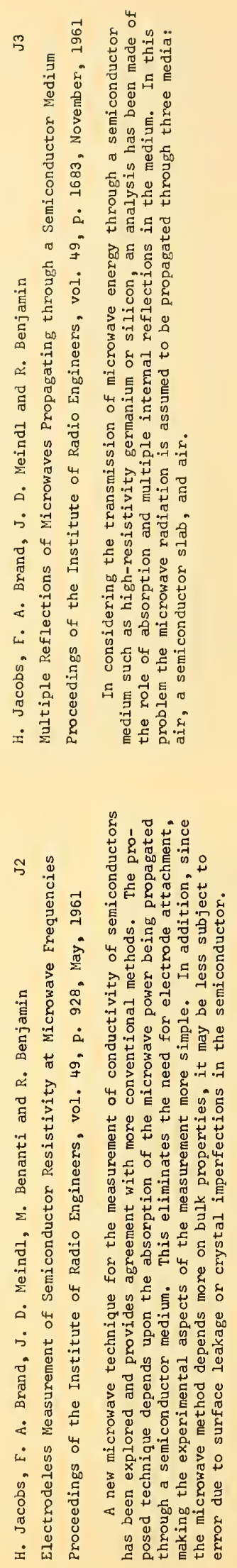

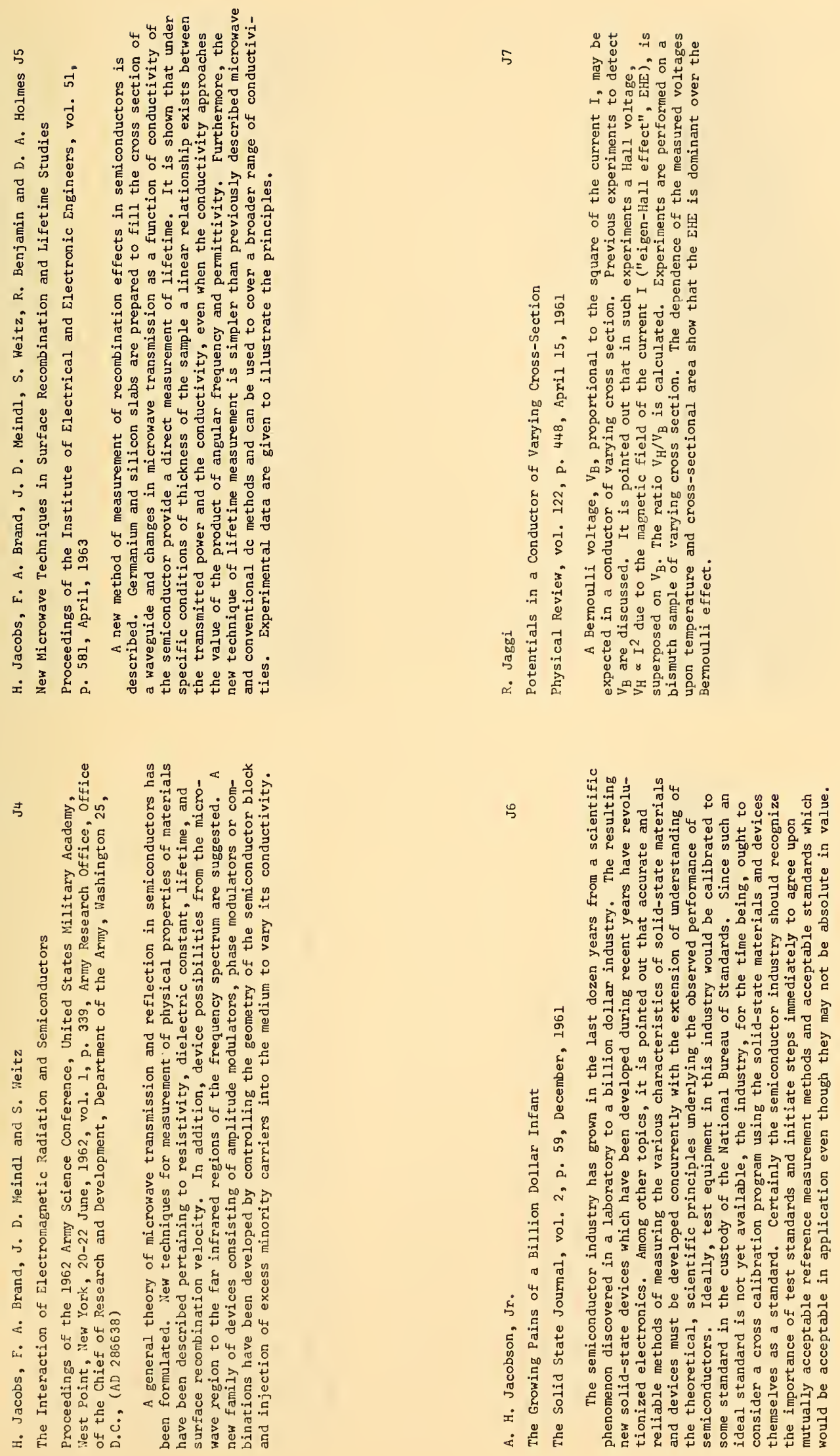

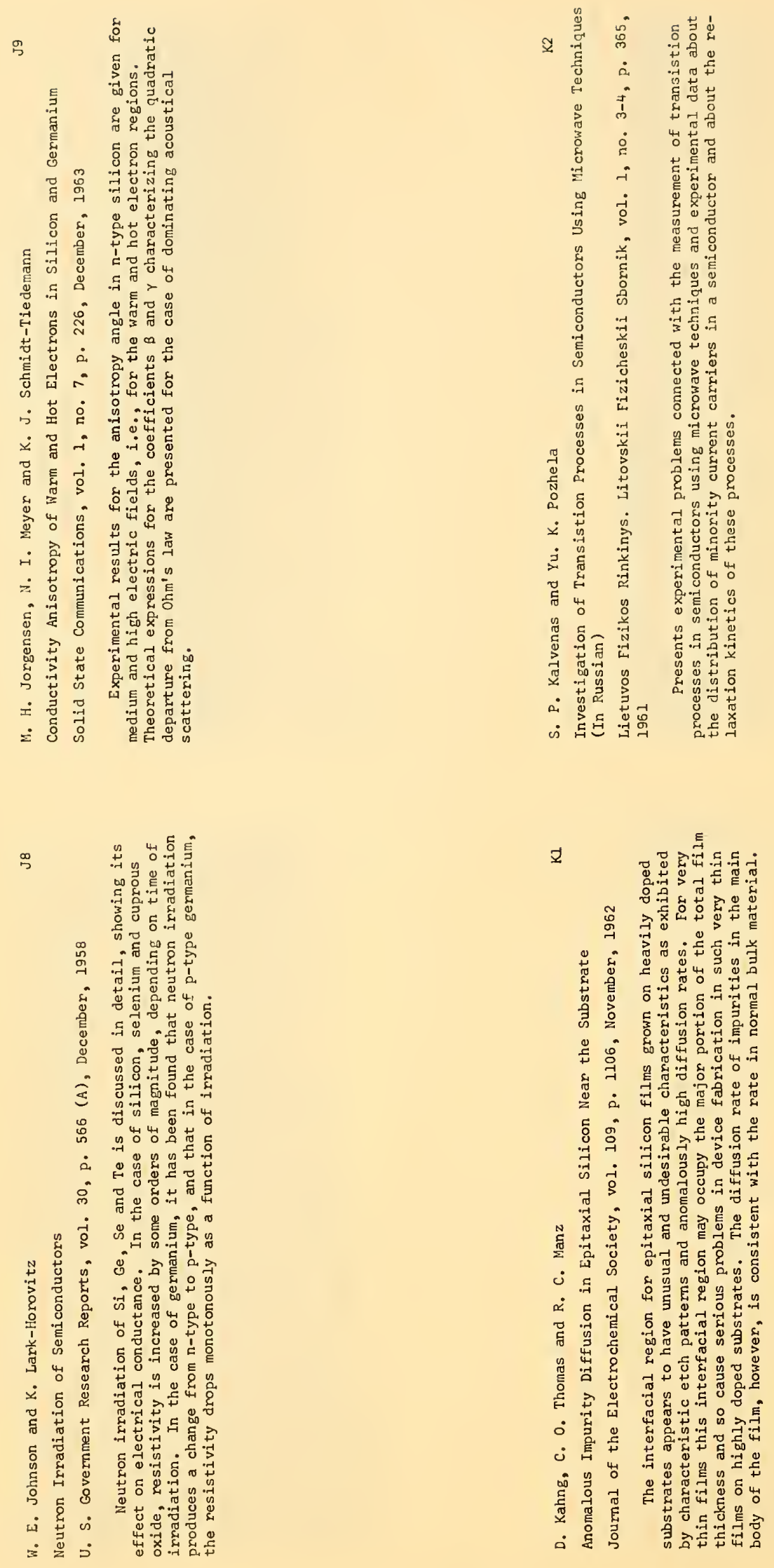

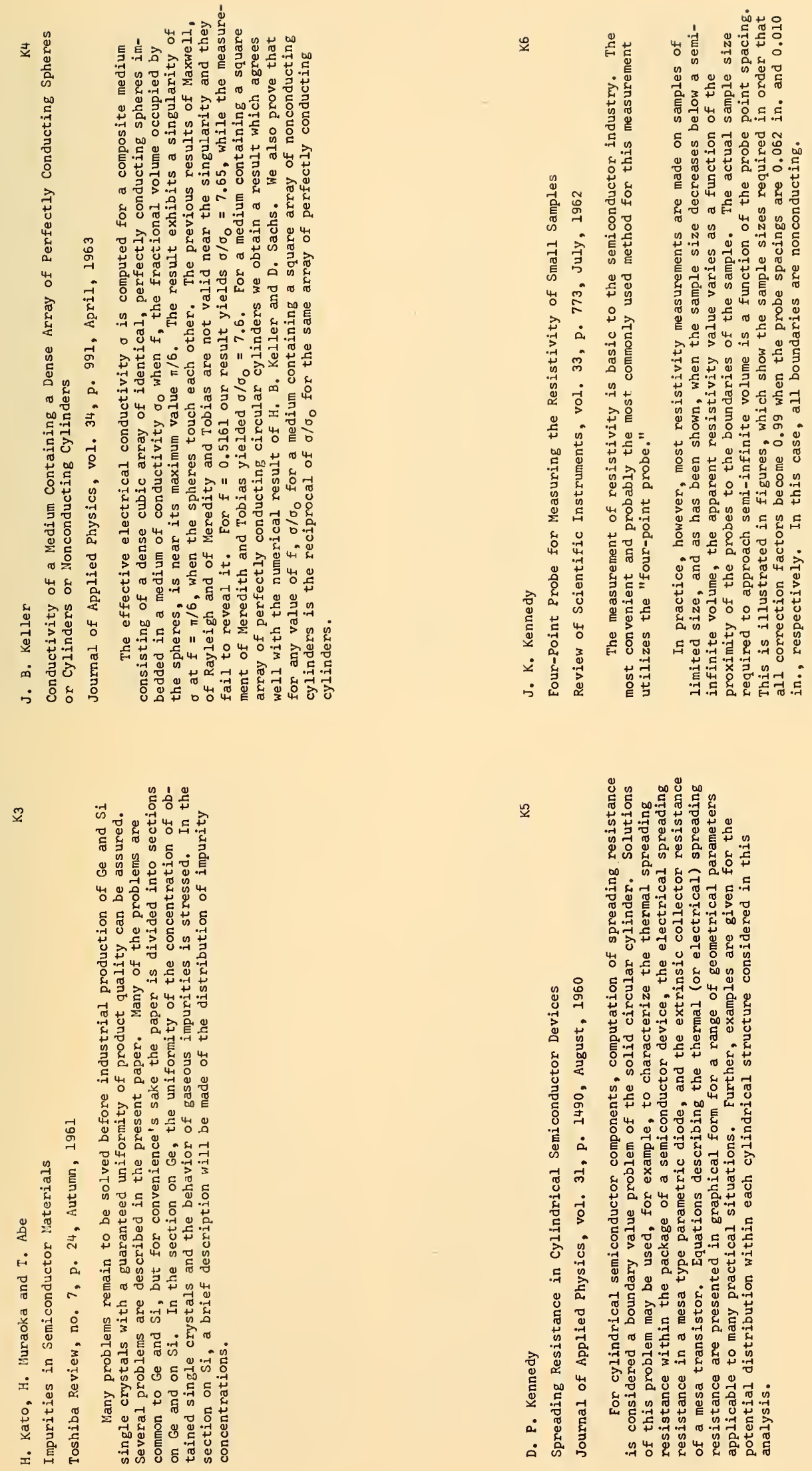

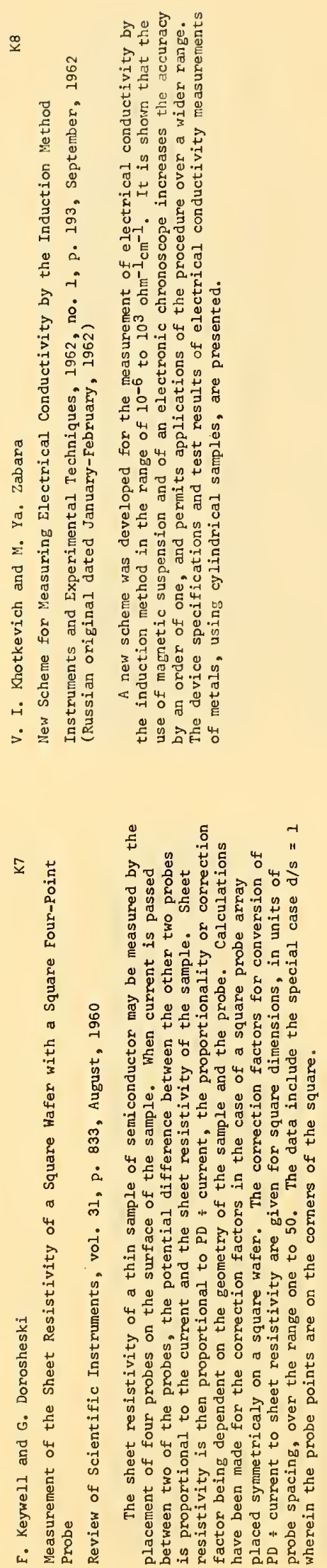
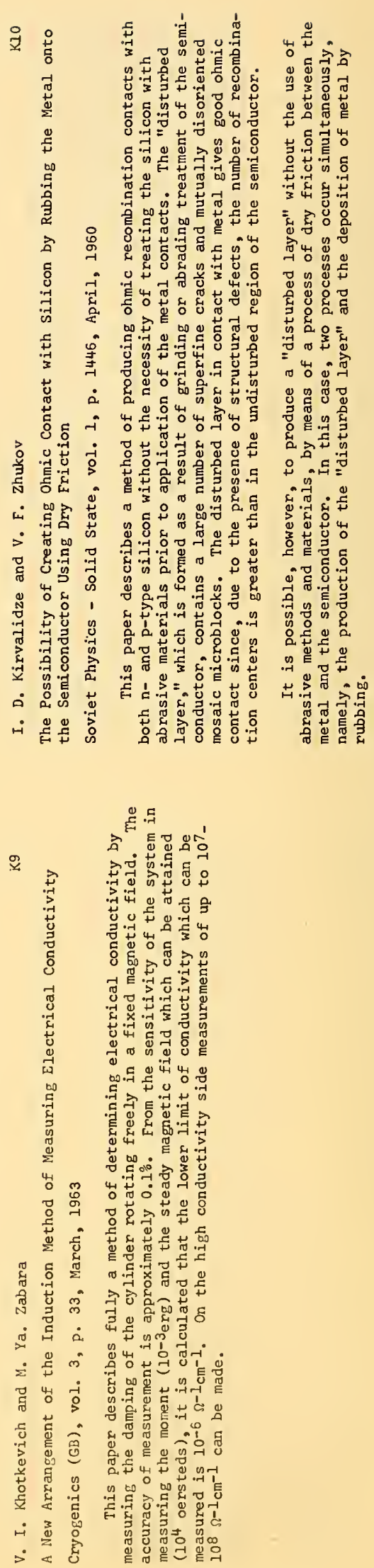

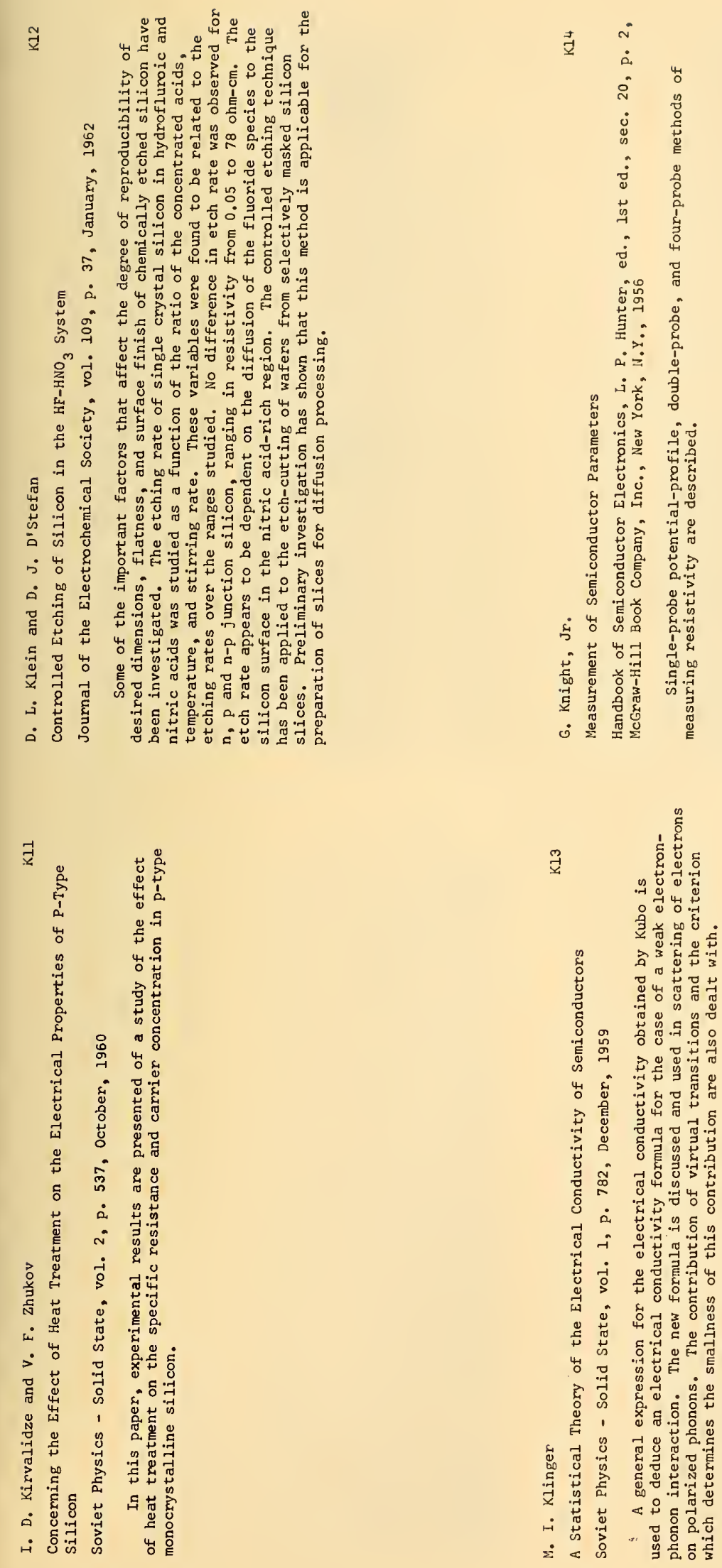

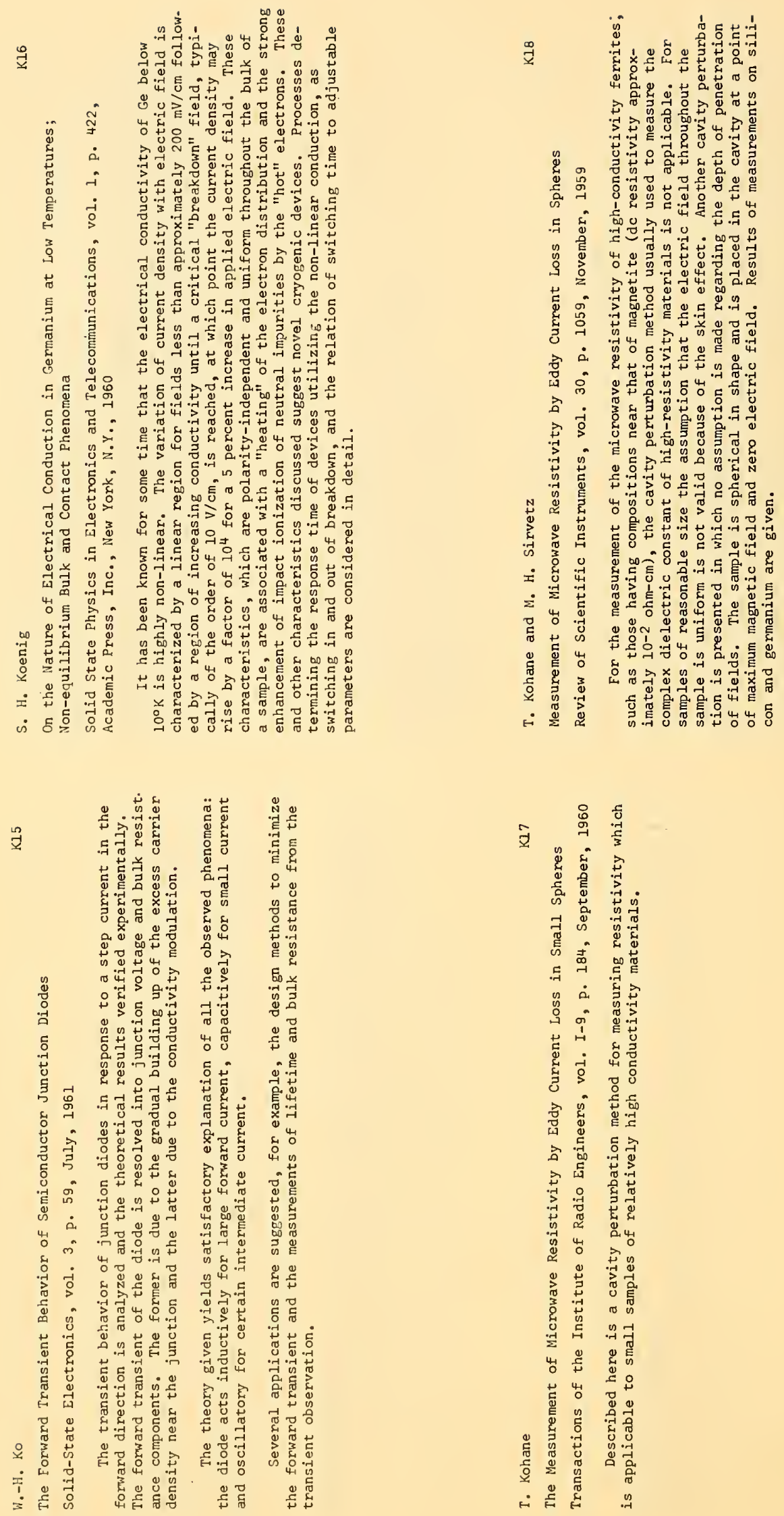

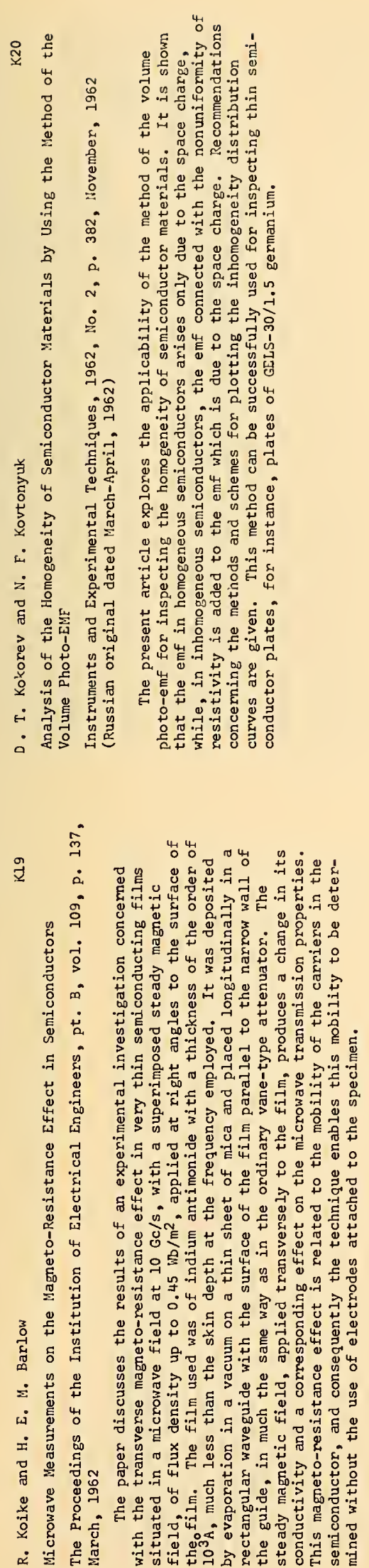
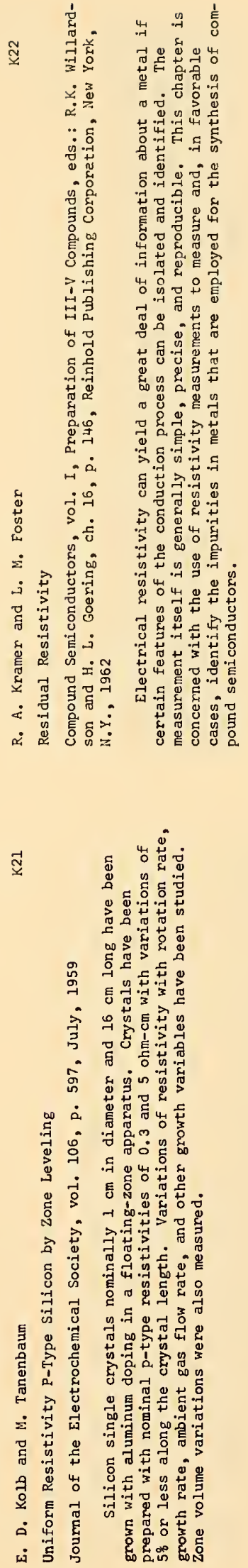

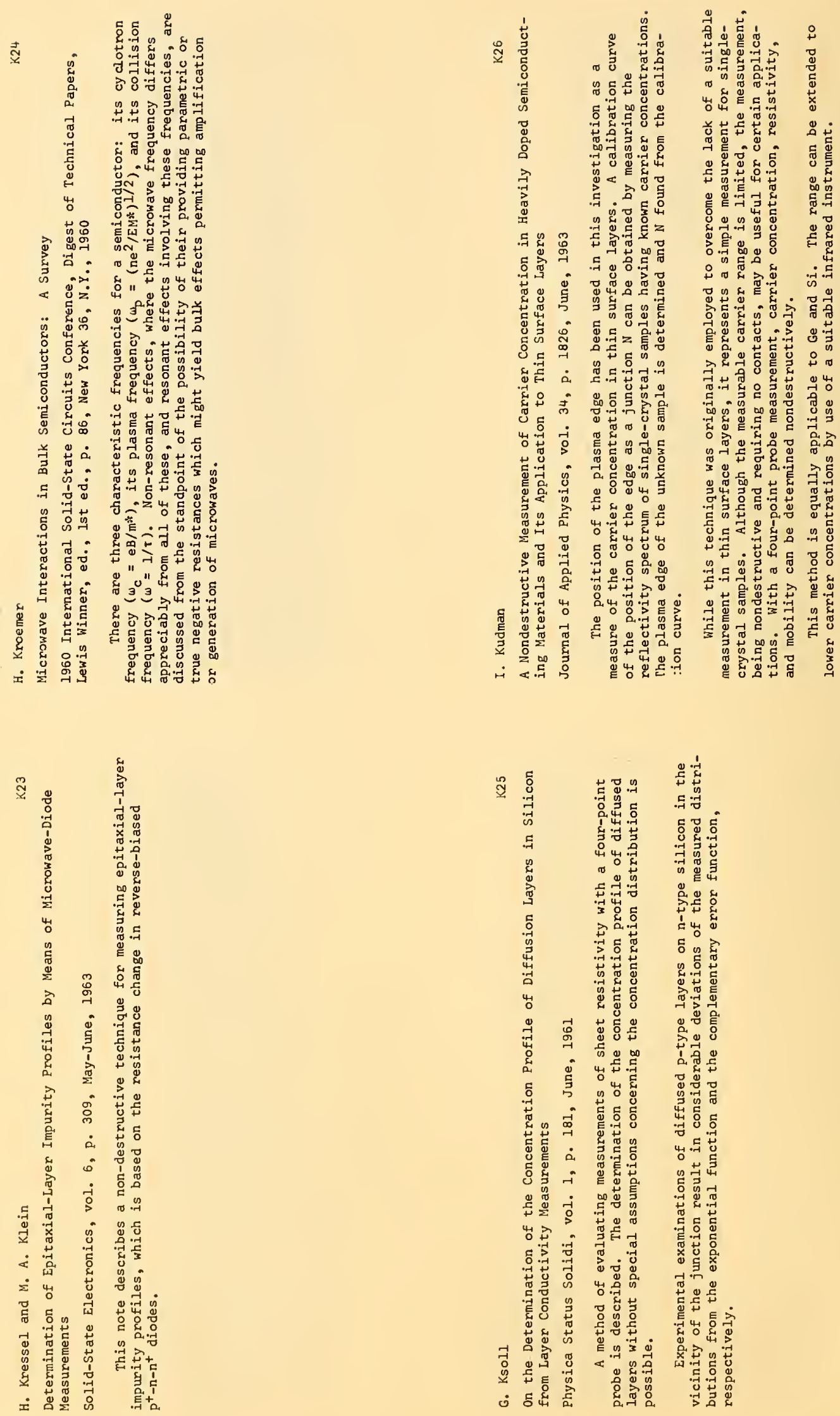

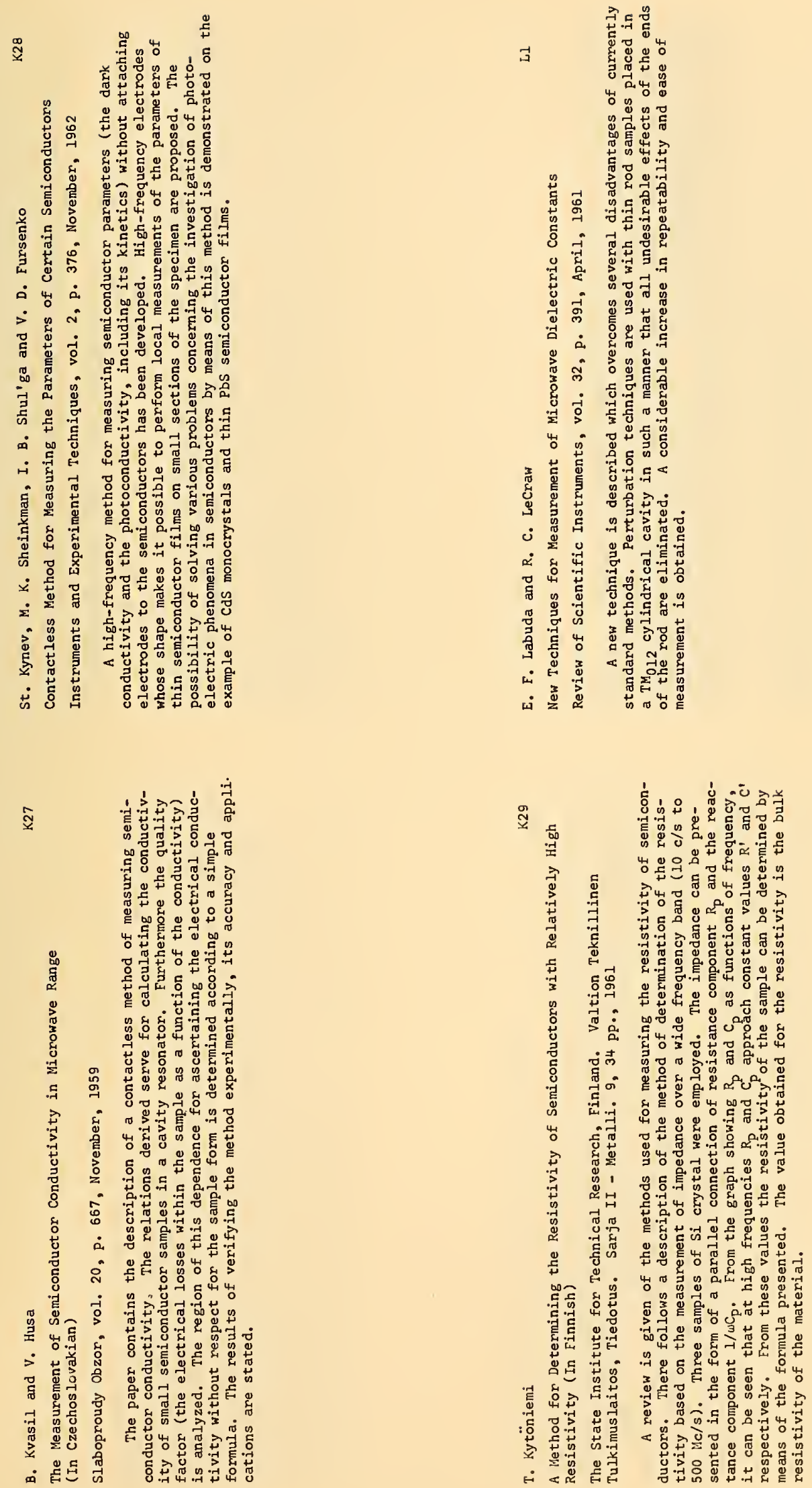

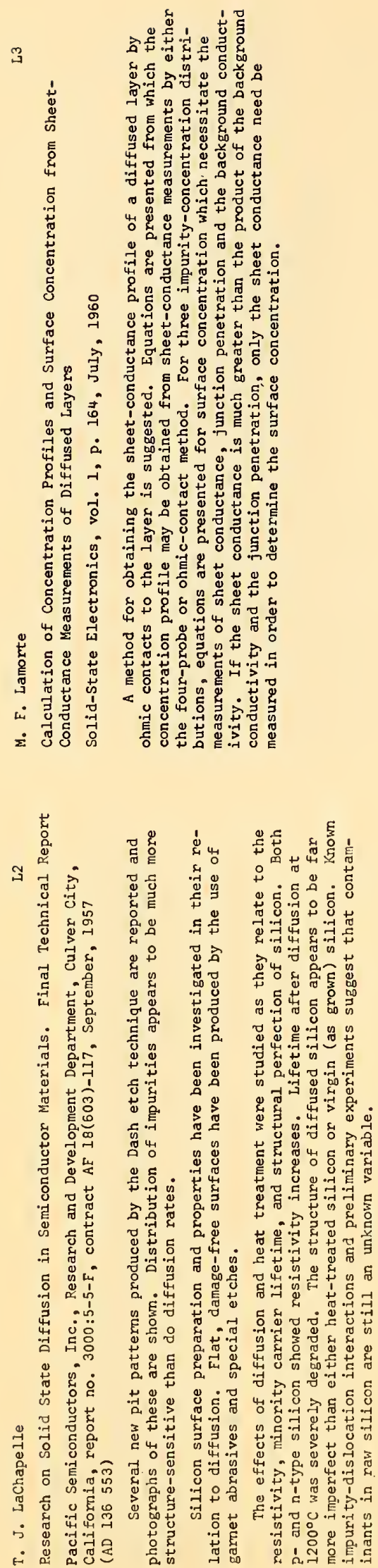
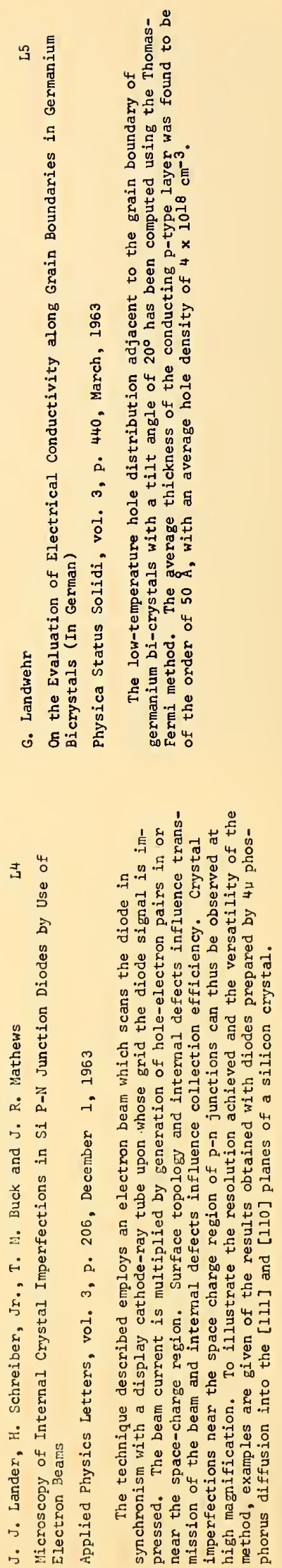


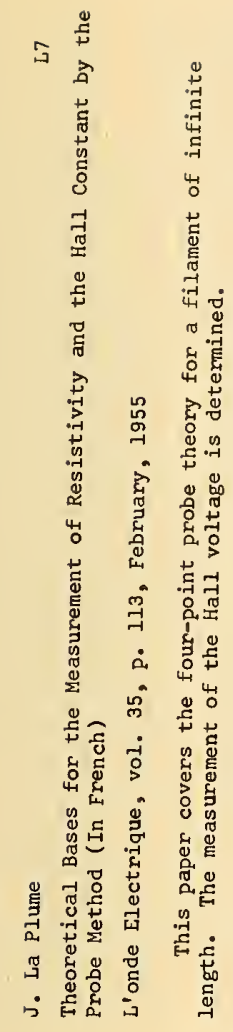

ำ

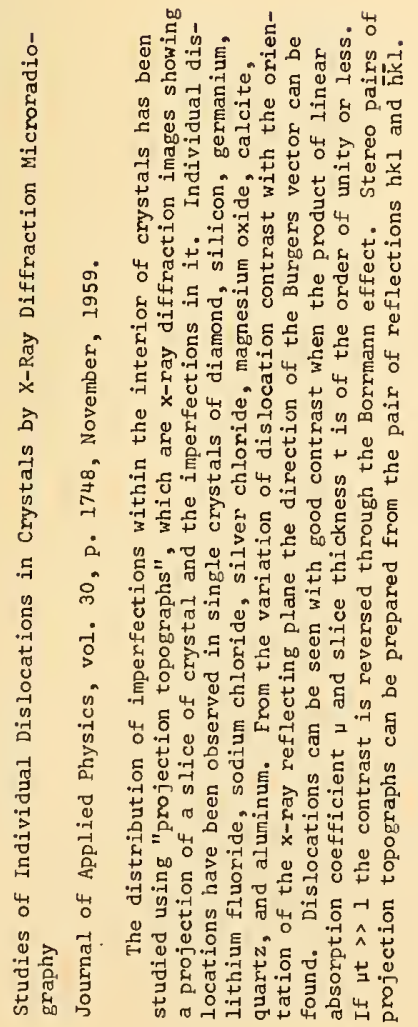

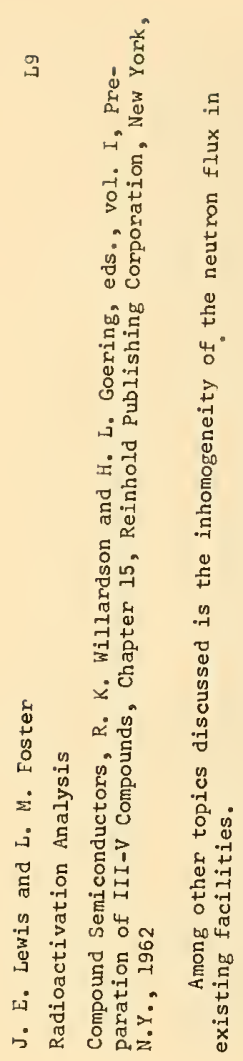

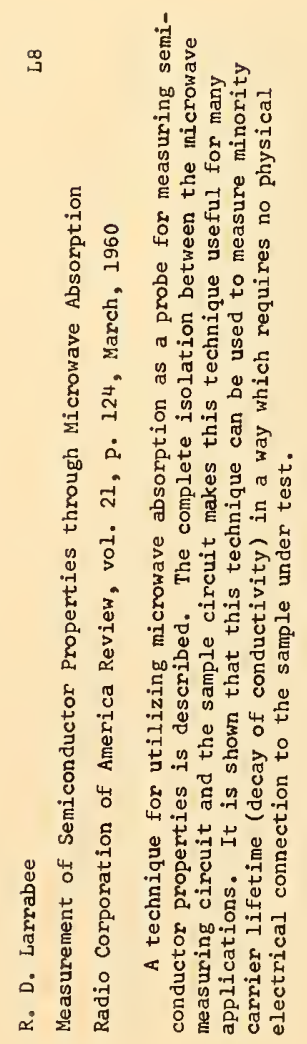



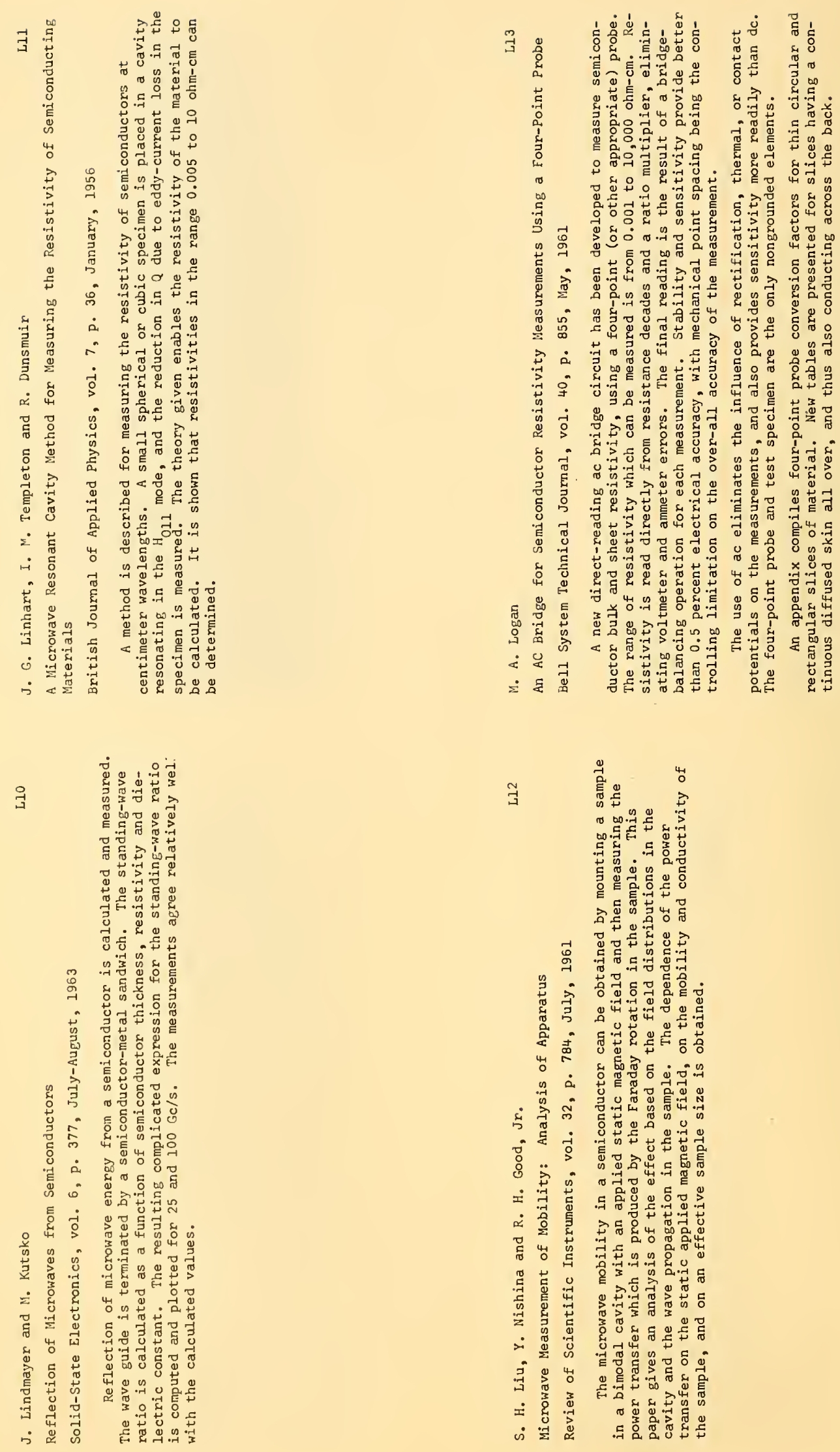

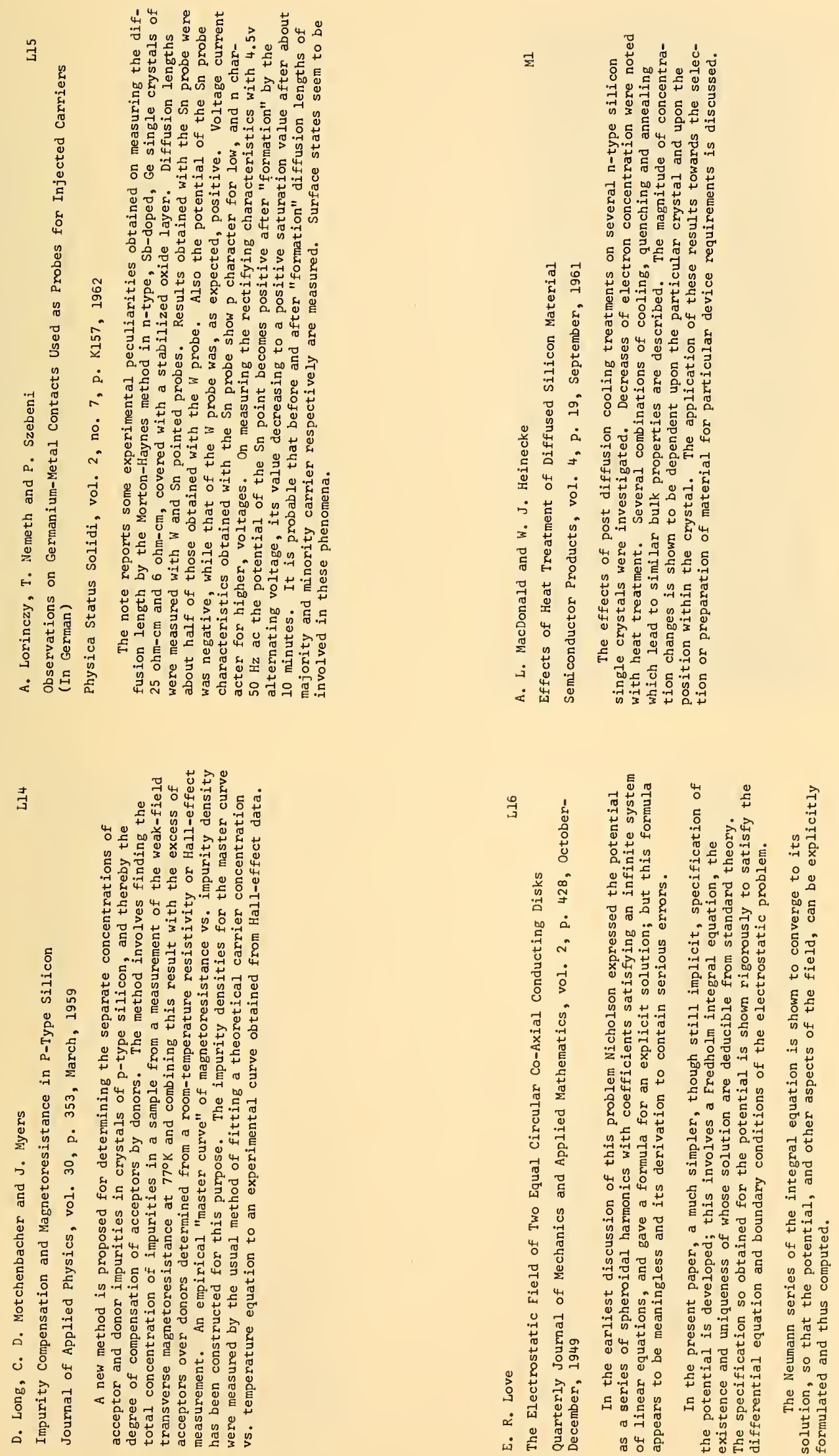

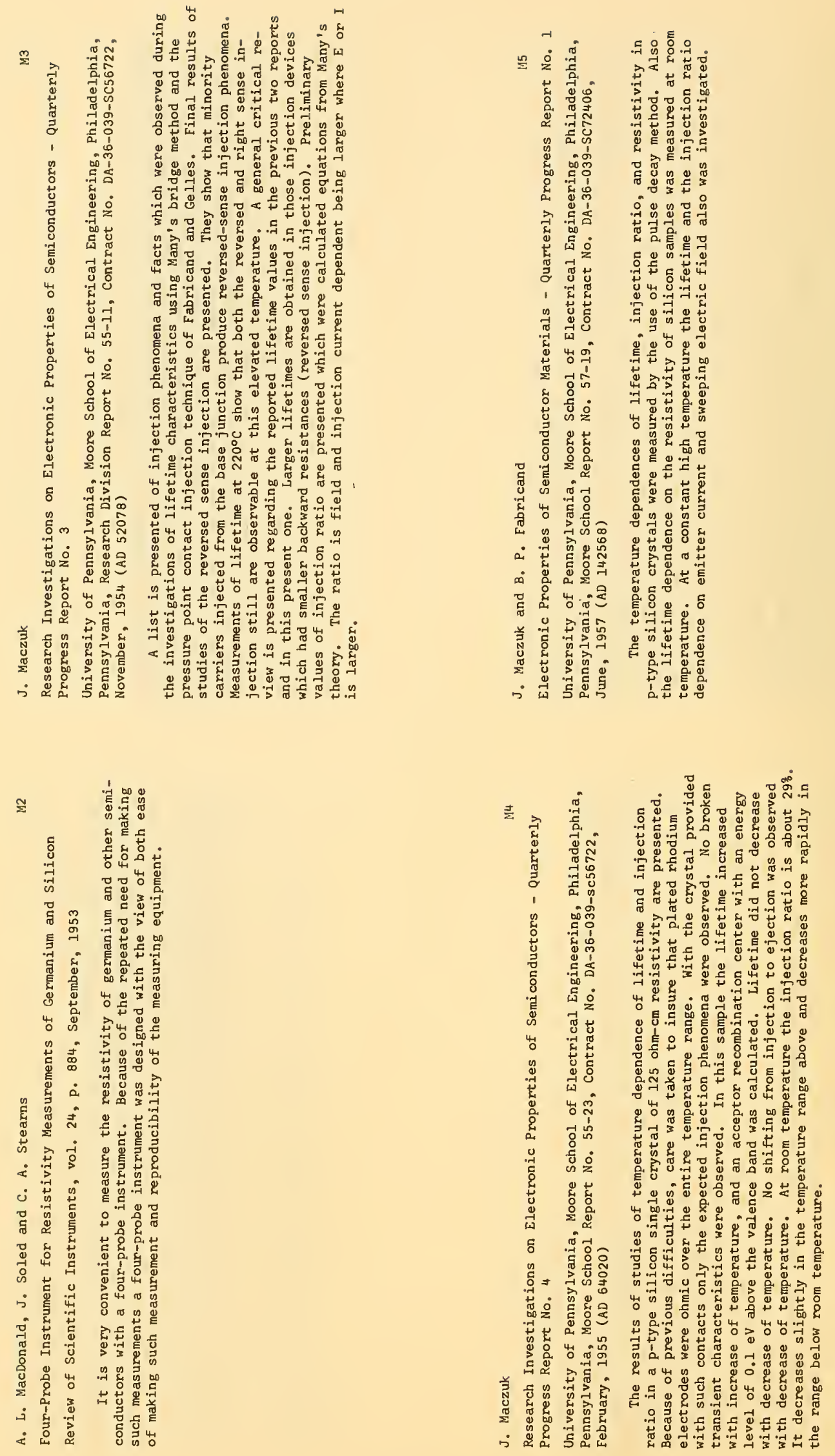

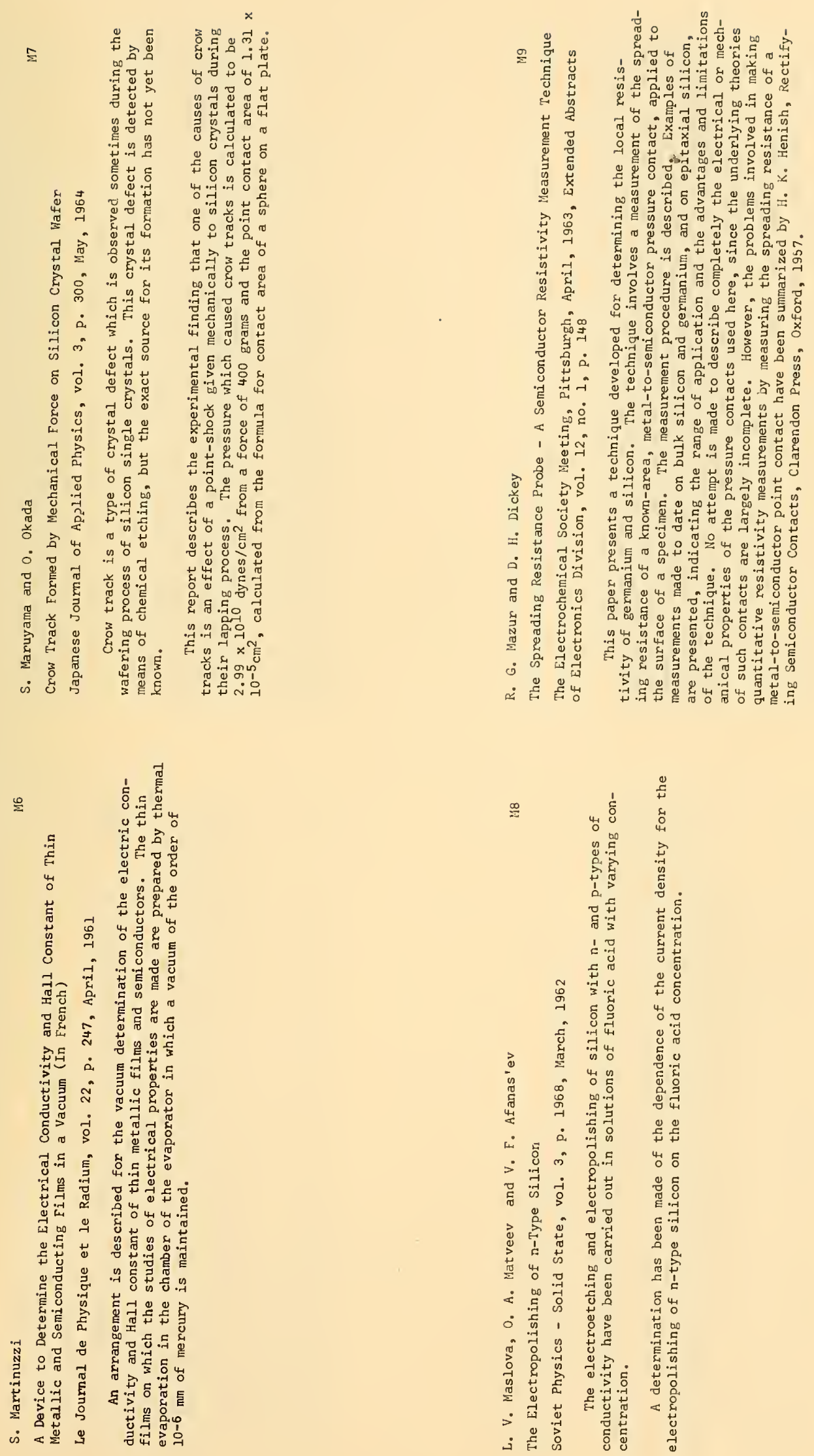

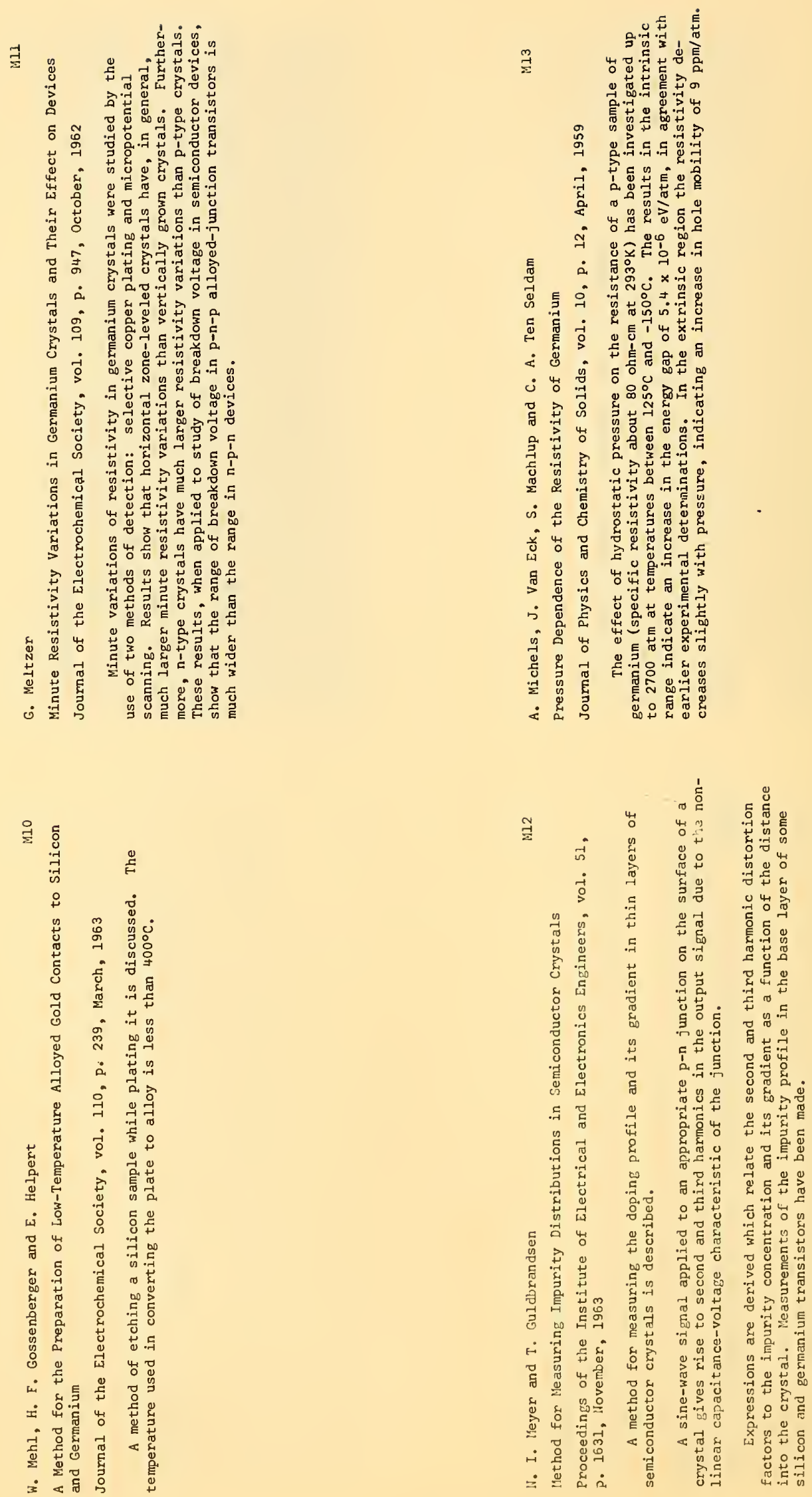

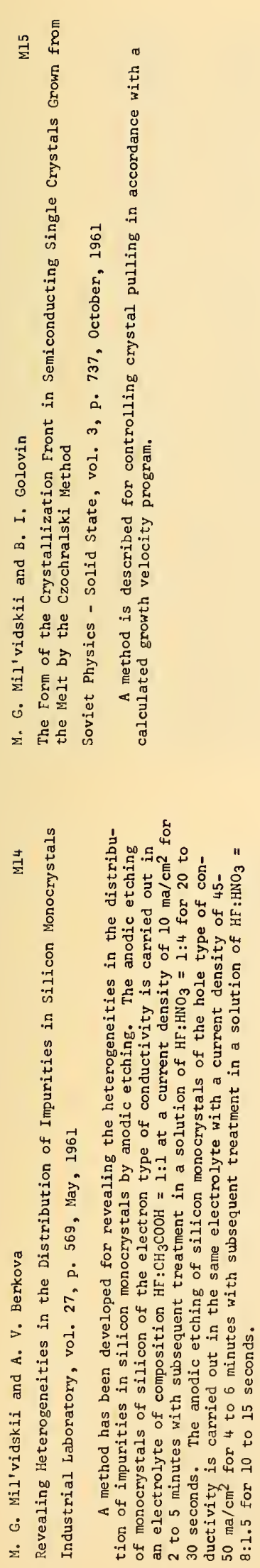

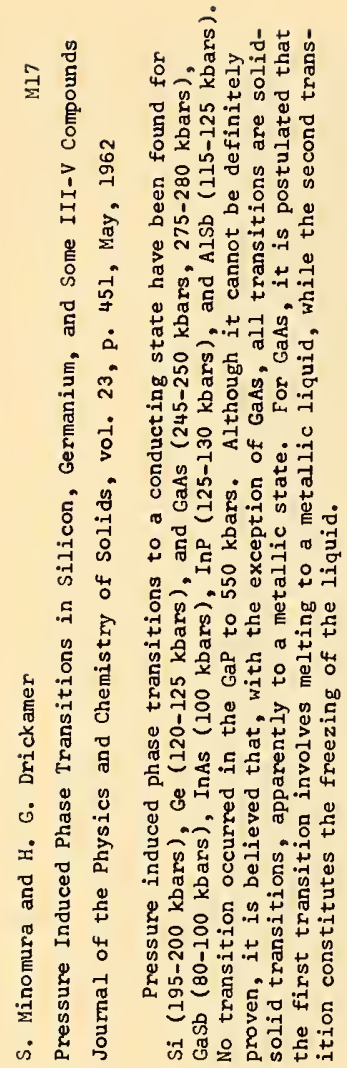

$\stackrel{0}{\Sigma}$

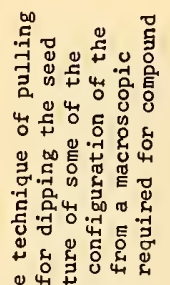

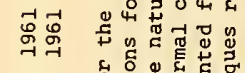

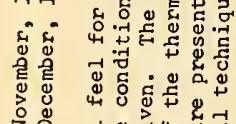

을

ผ

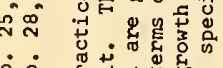

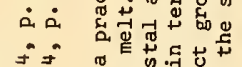

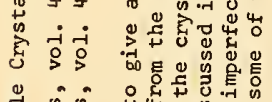

Do

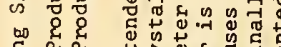

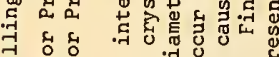

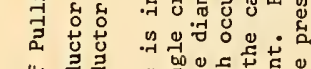

虫 考古

它

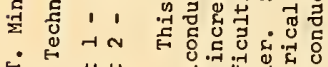

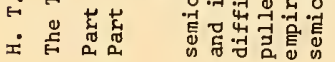



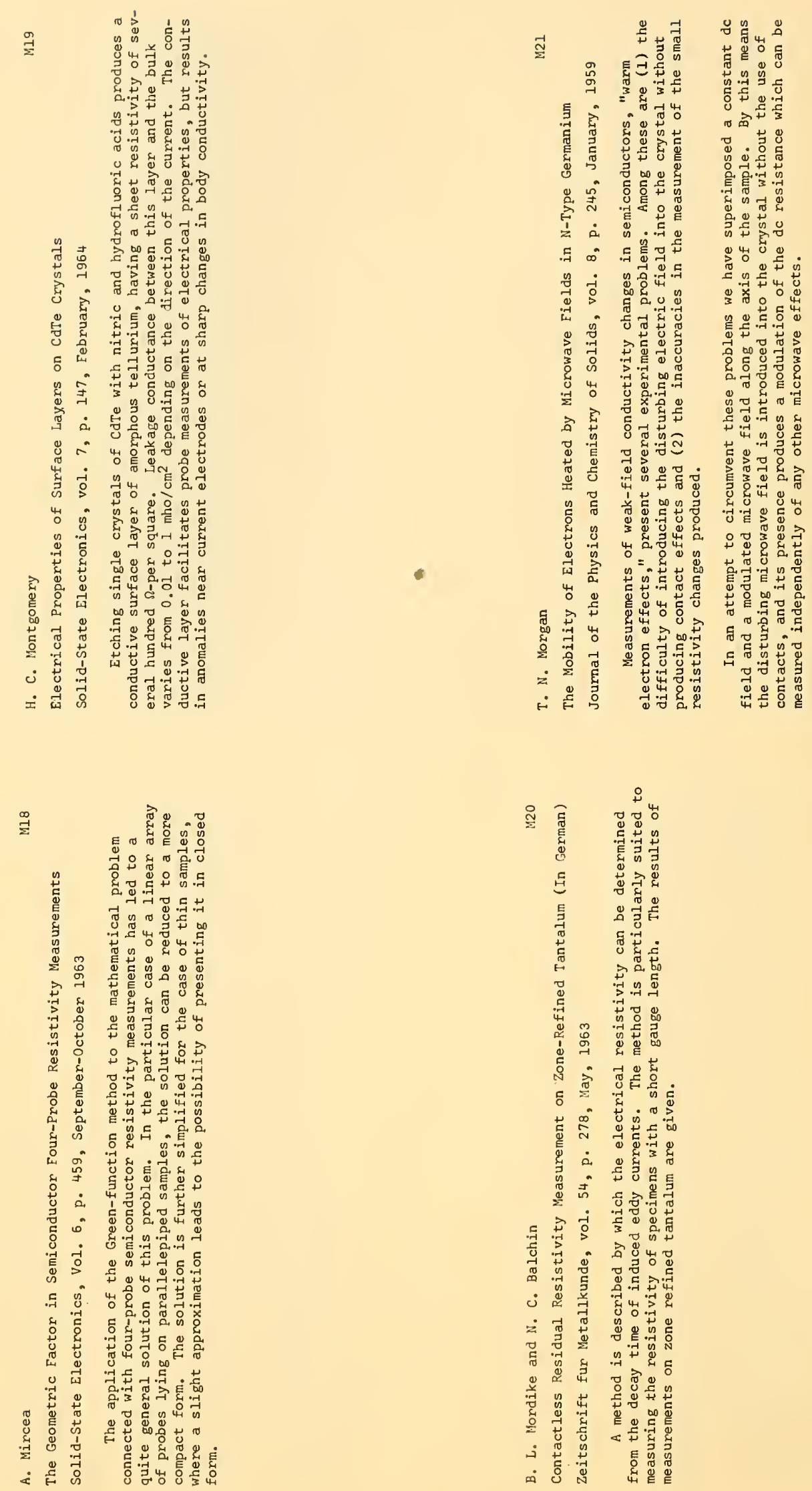


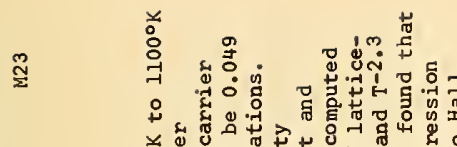

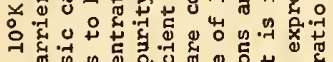

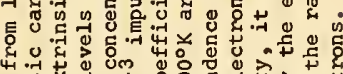

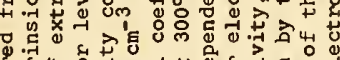

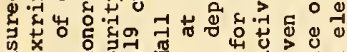

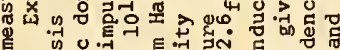

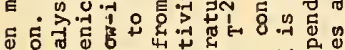

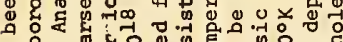

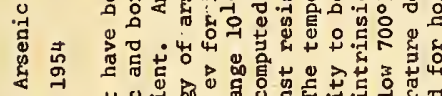
足 -100

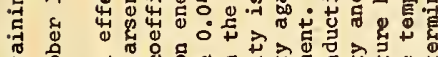

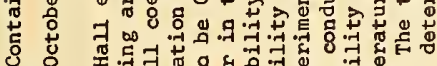

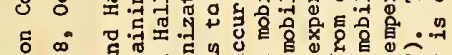

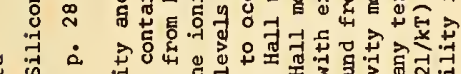
ग-

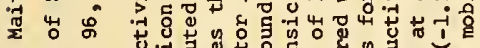

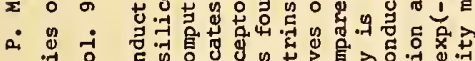

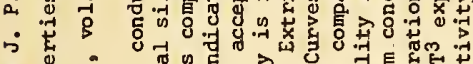

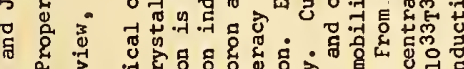

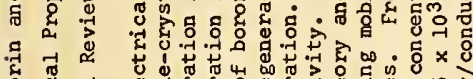
至范

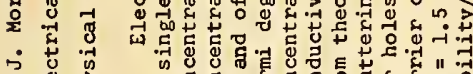

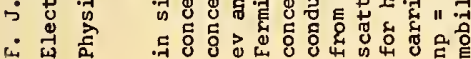
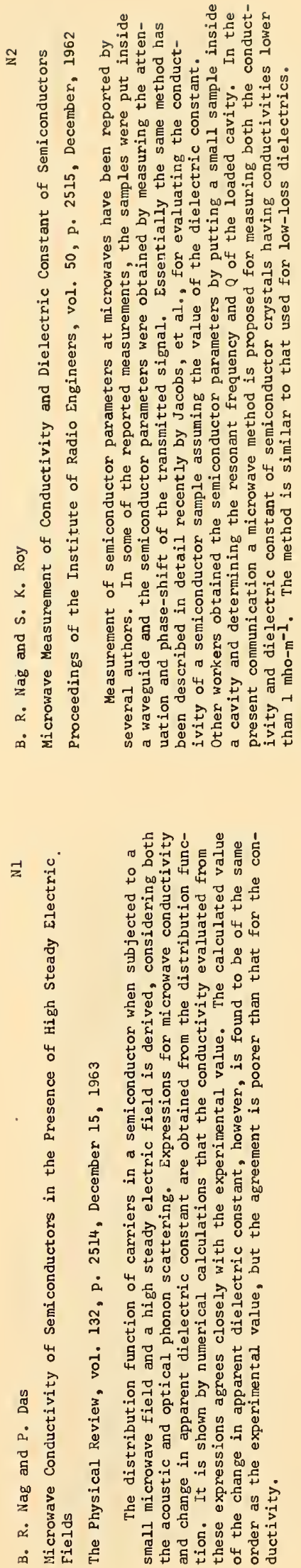

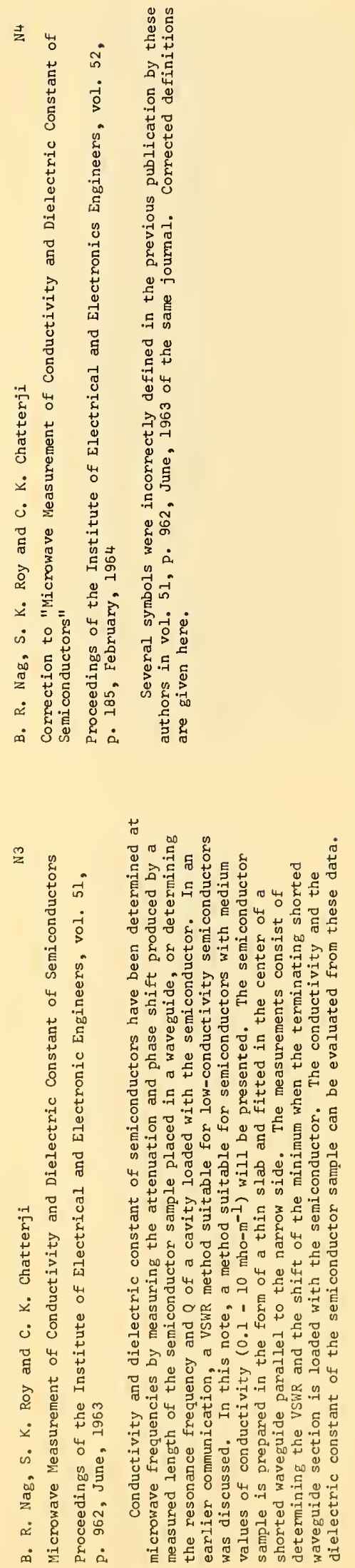
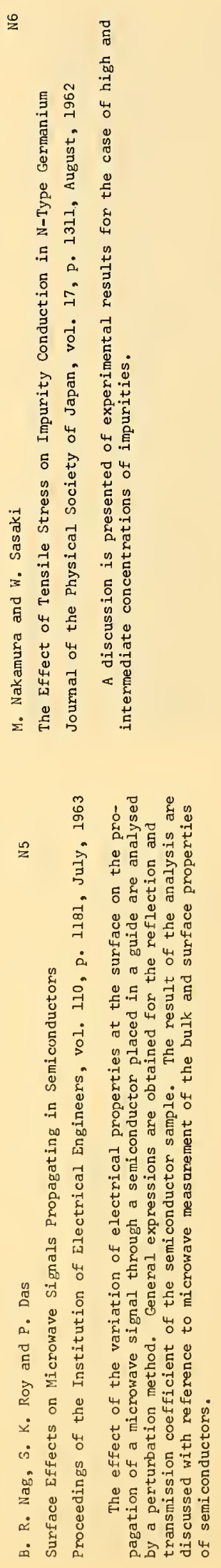

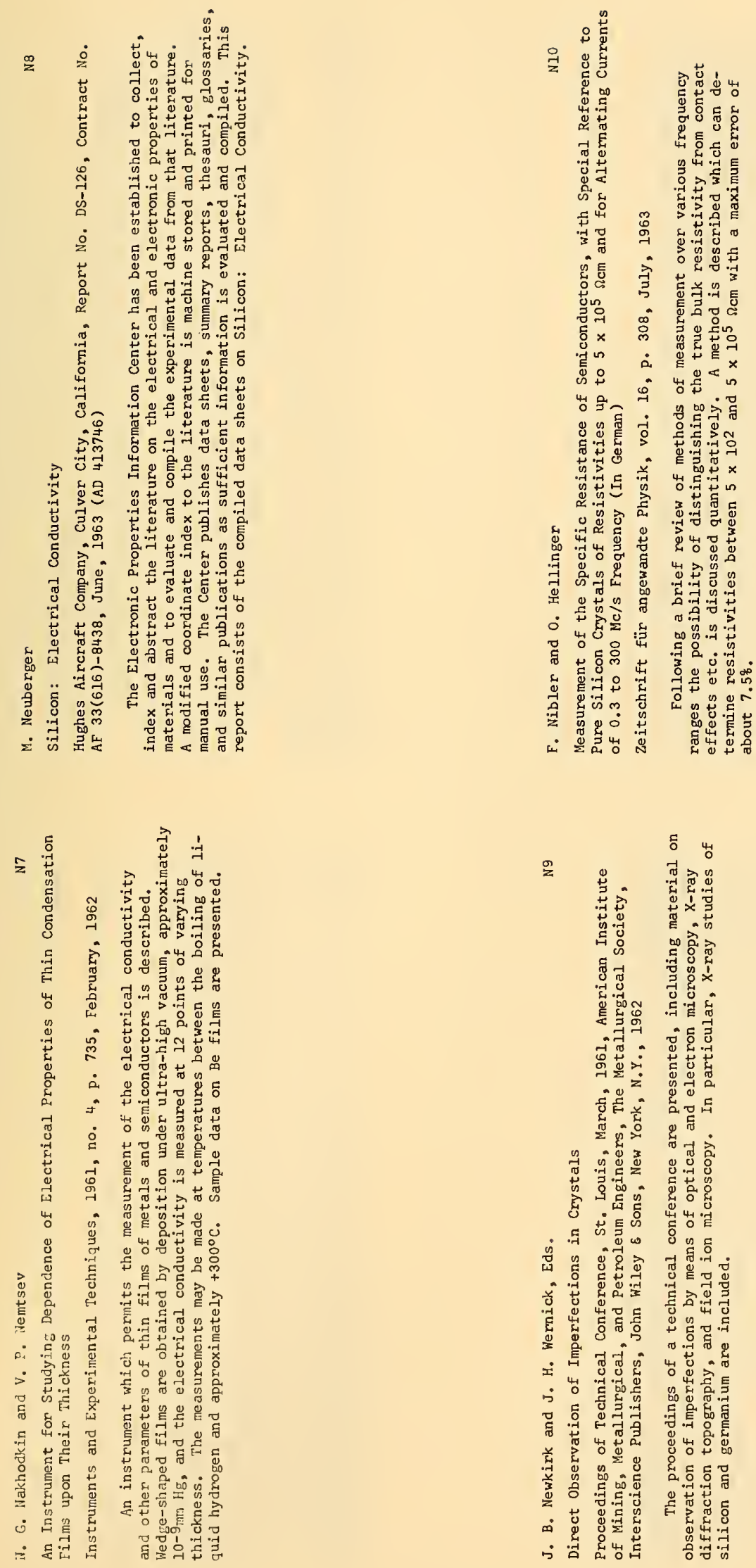

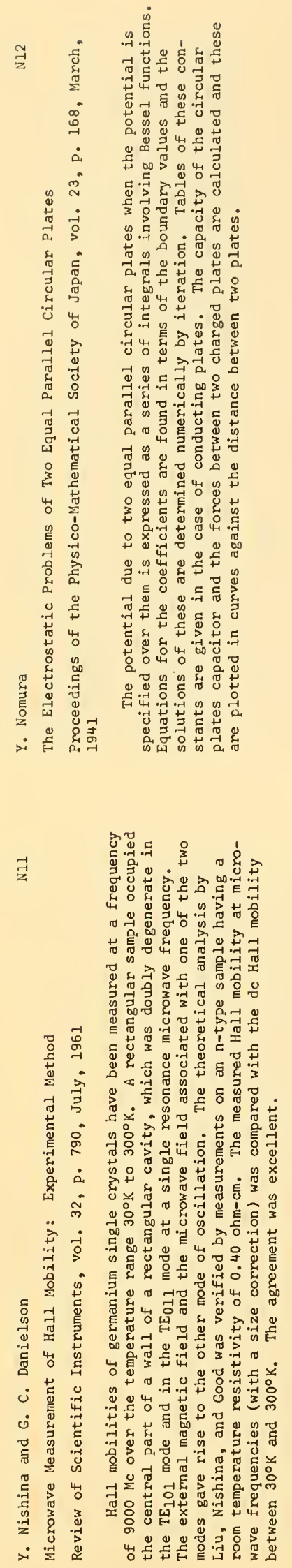
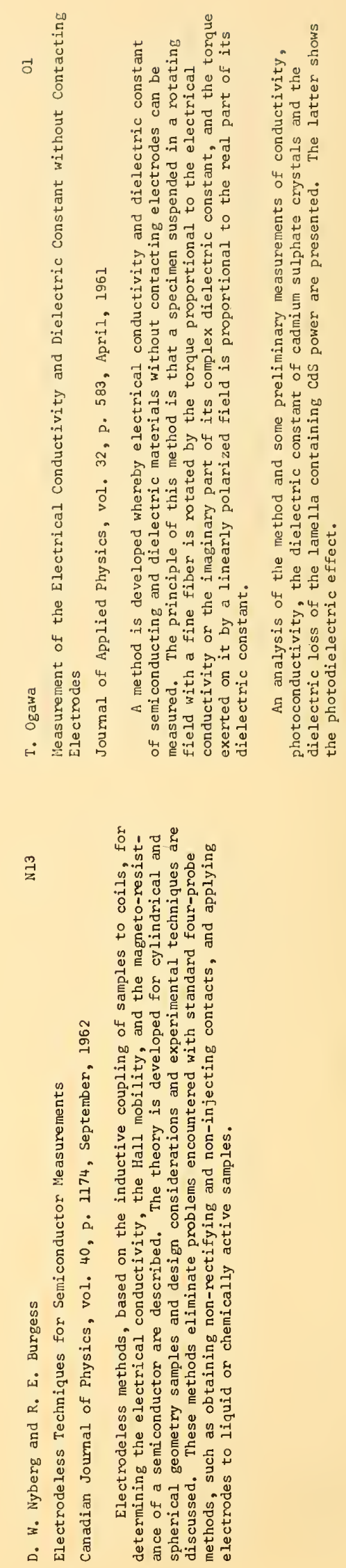


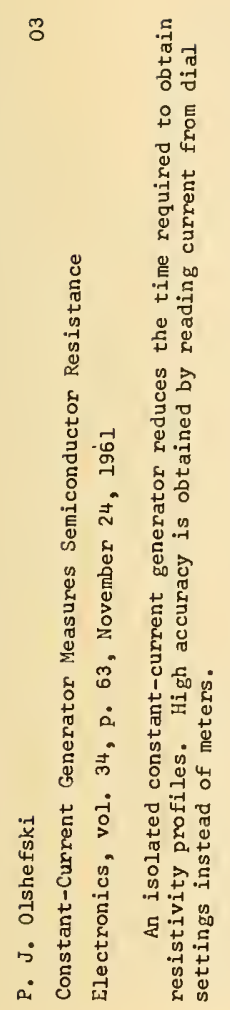

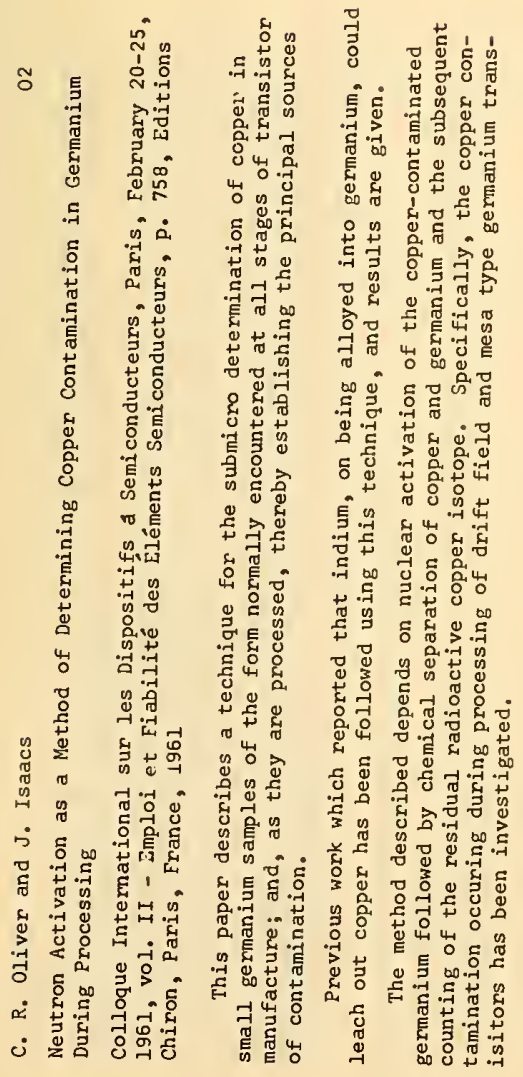
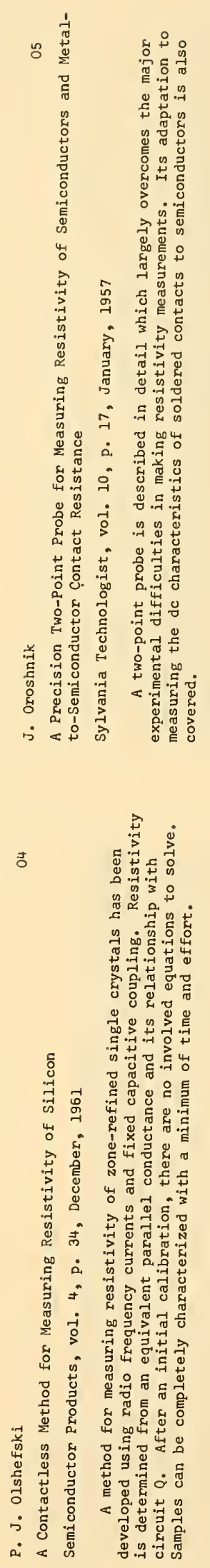

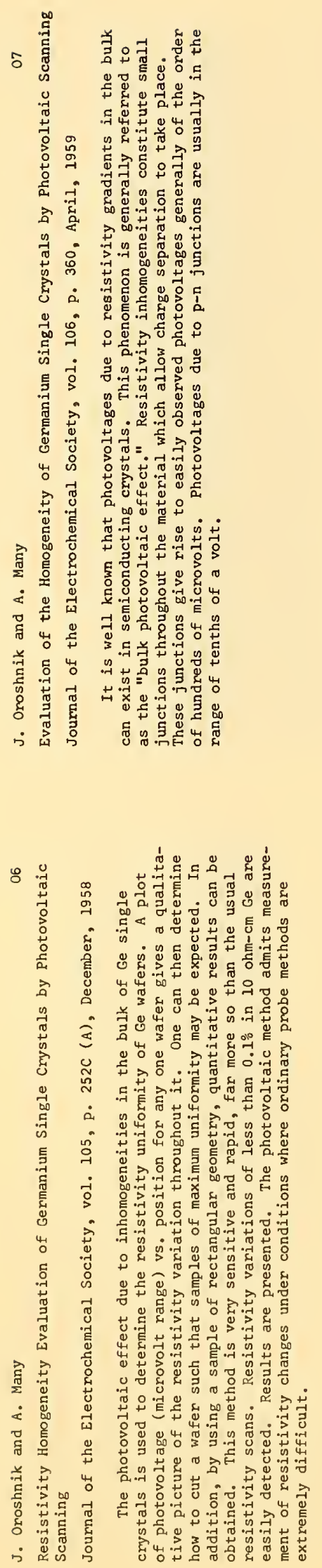
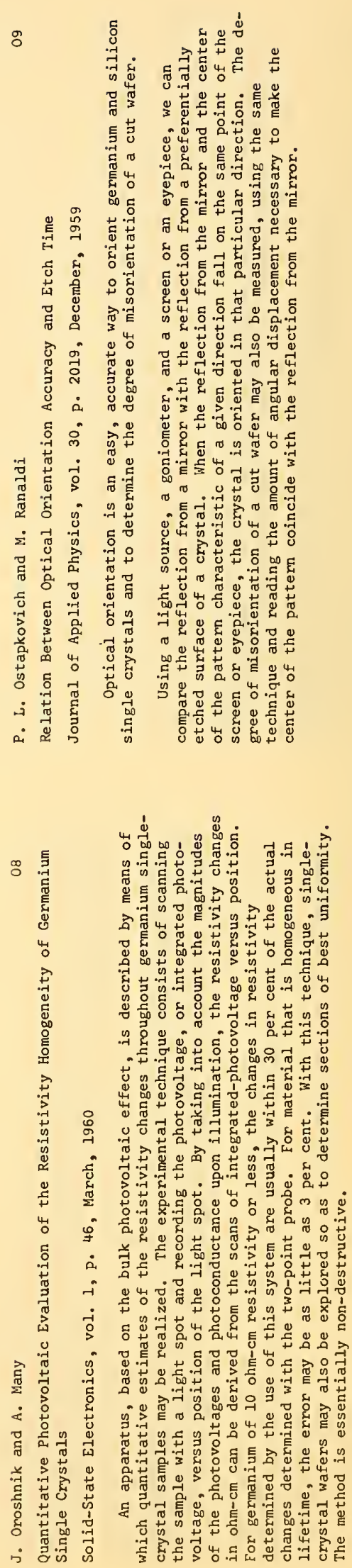

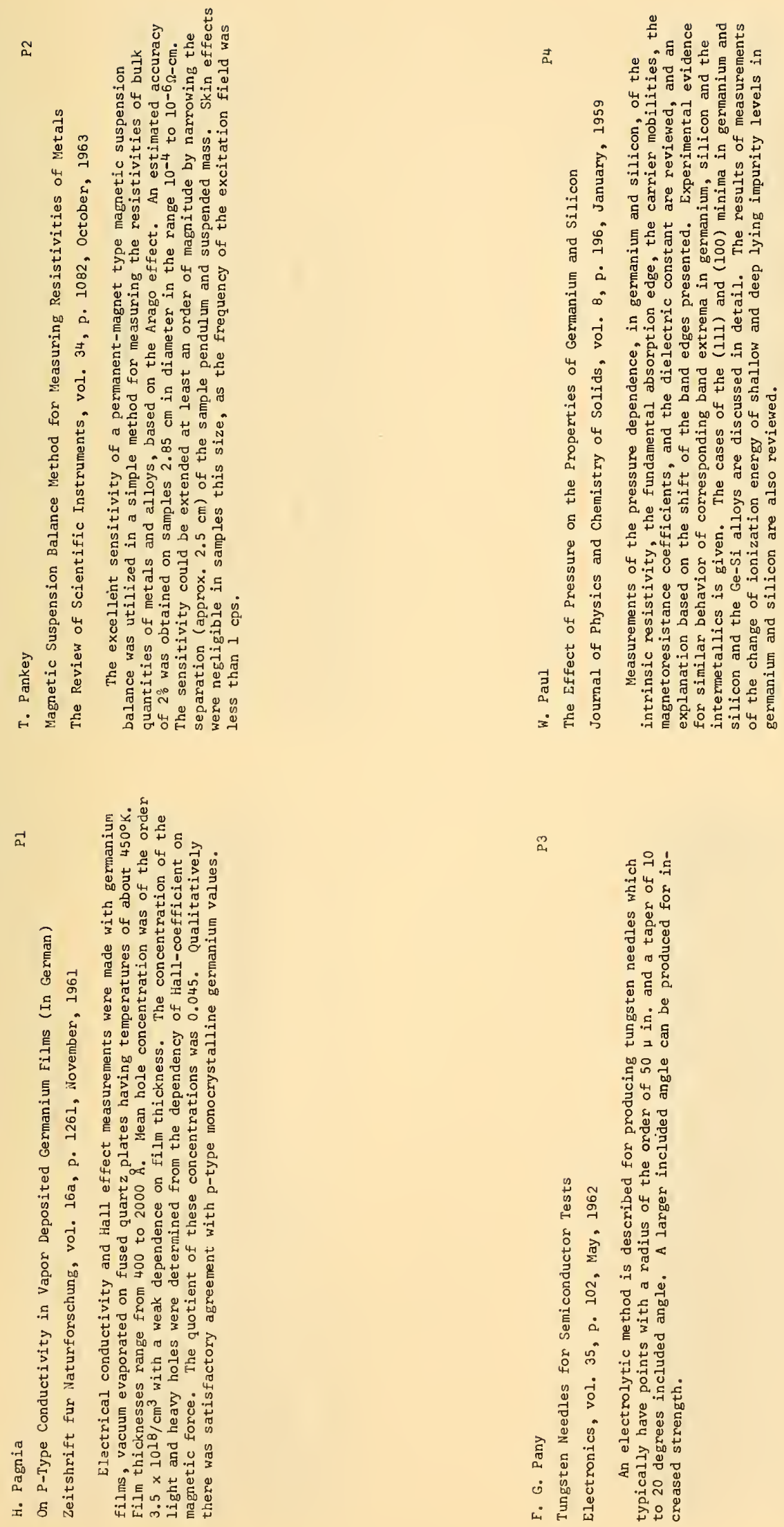

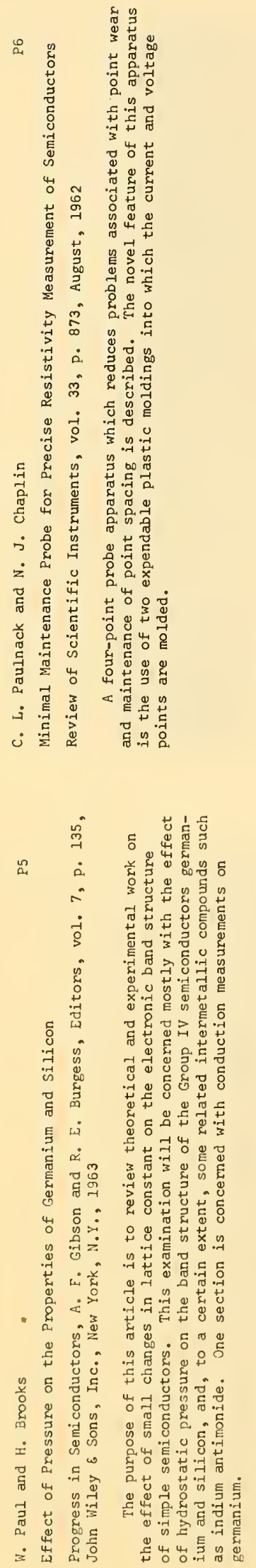
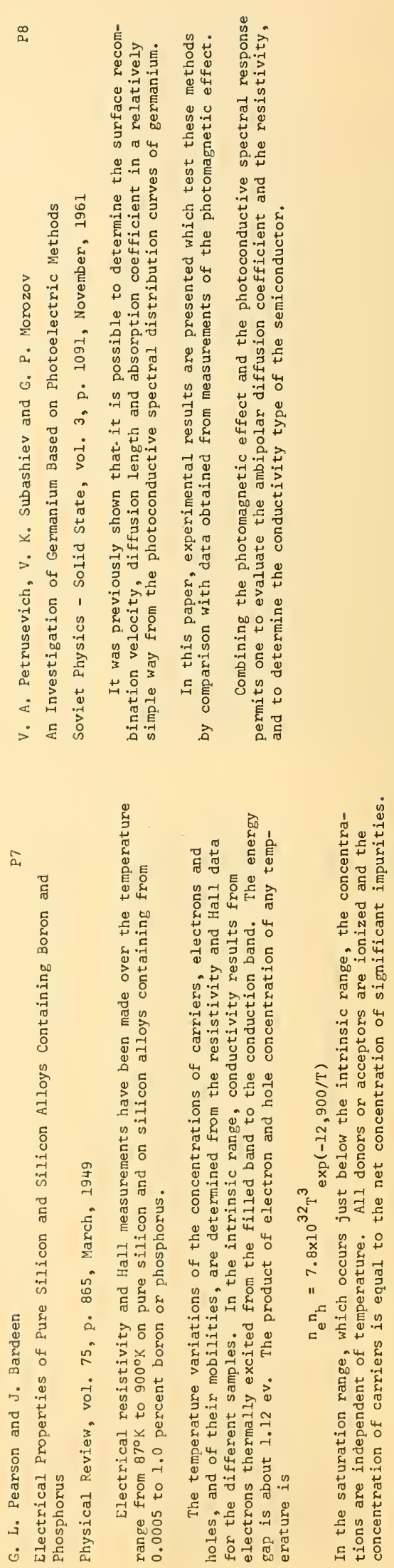

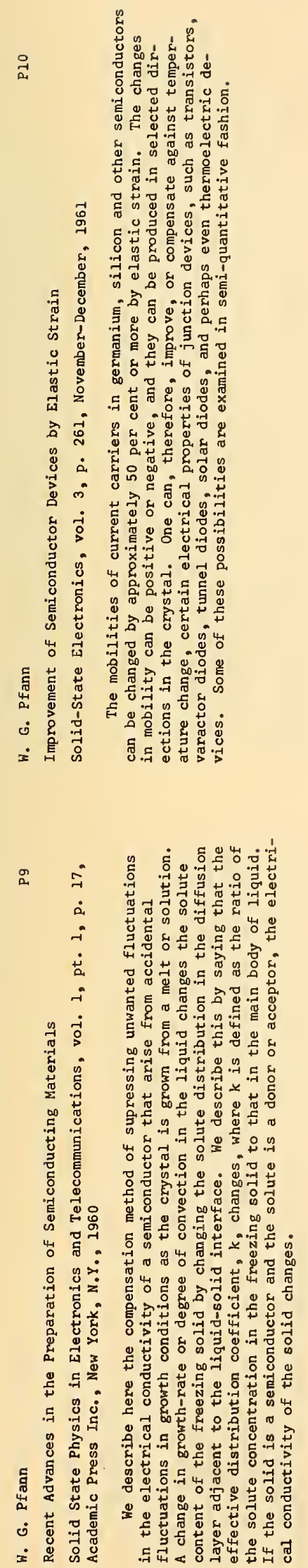
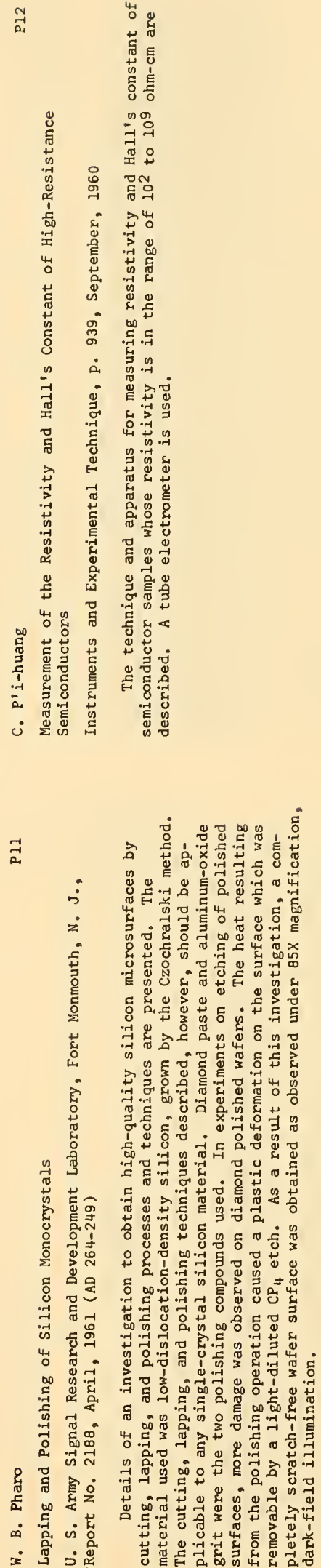

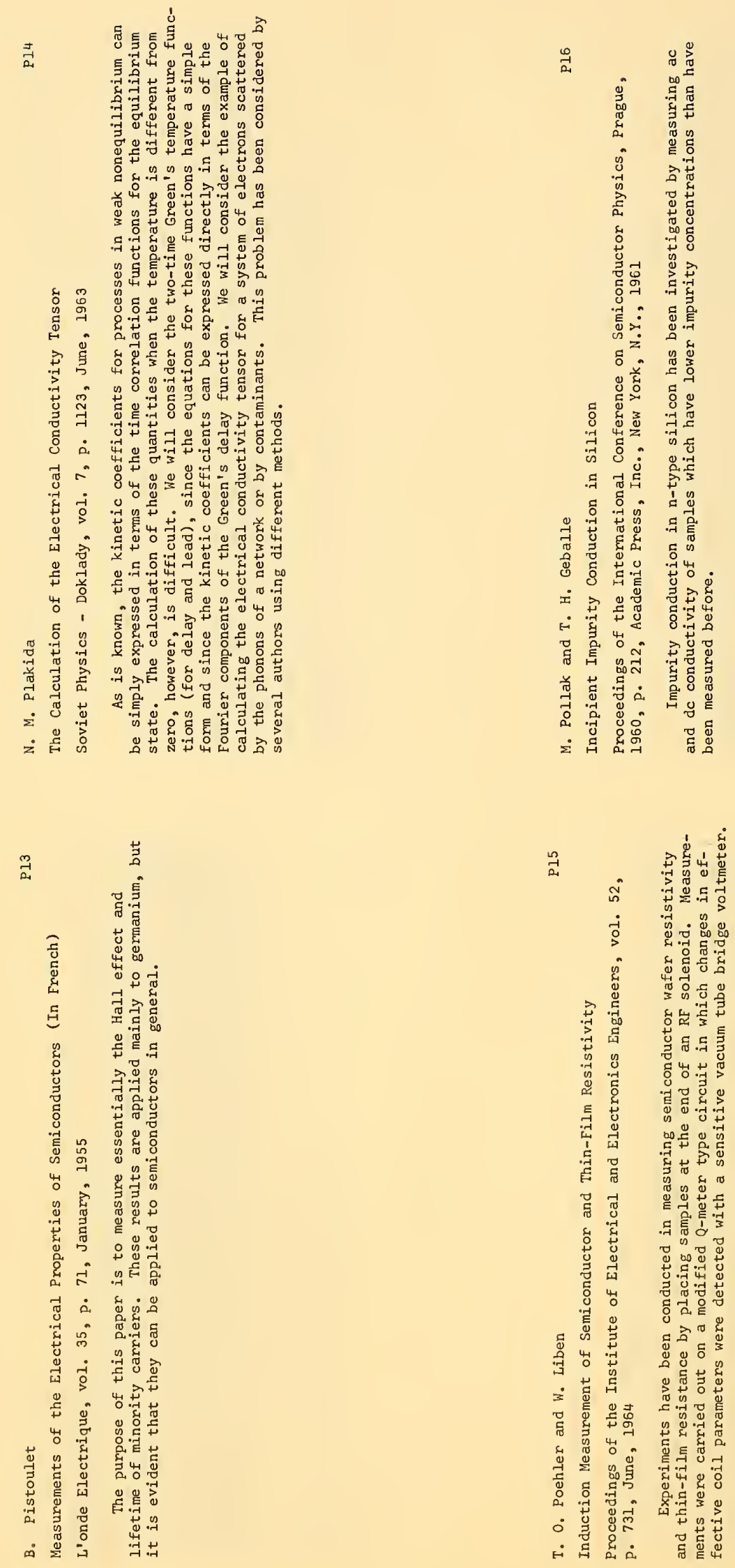


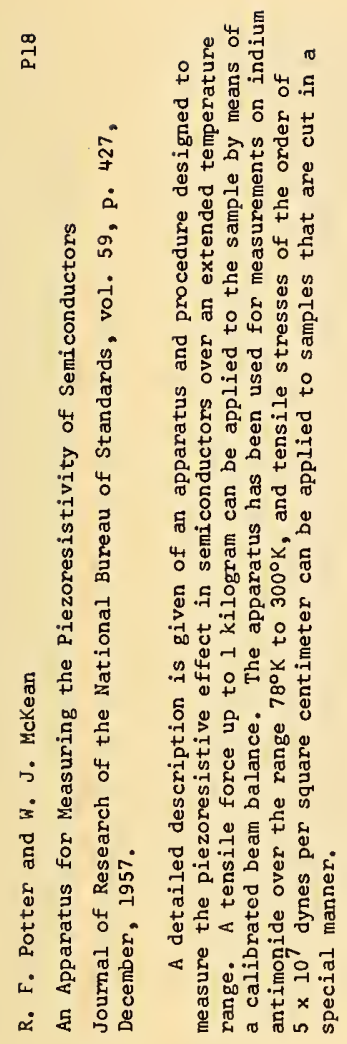

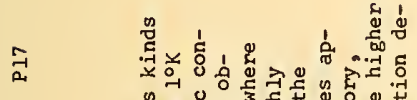

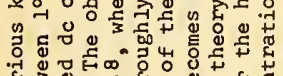

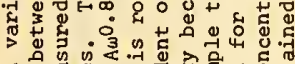

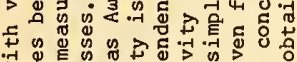

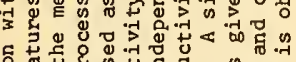

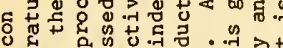

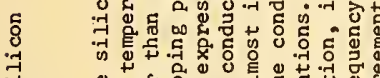

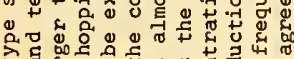

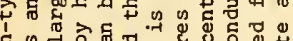

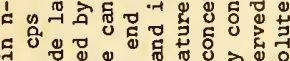

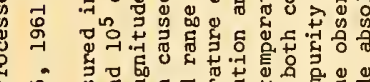

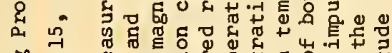

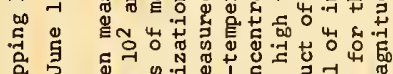

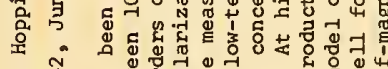

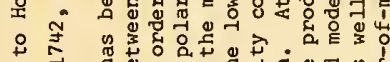

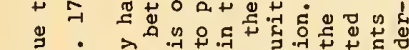

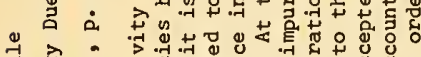

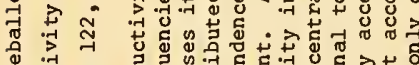

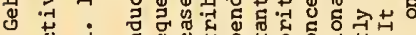

×

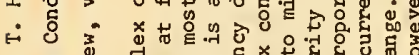

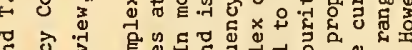

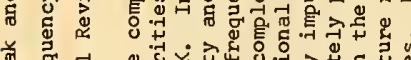

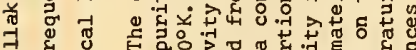
당

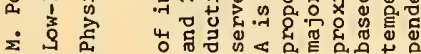
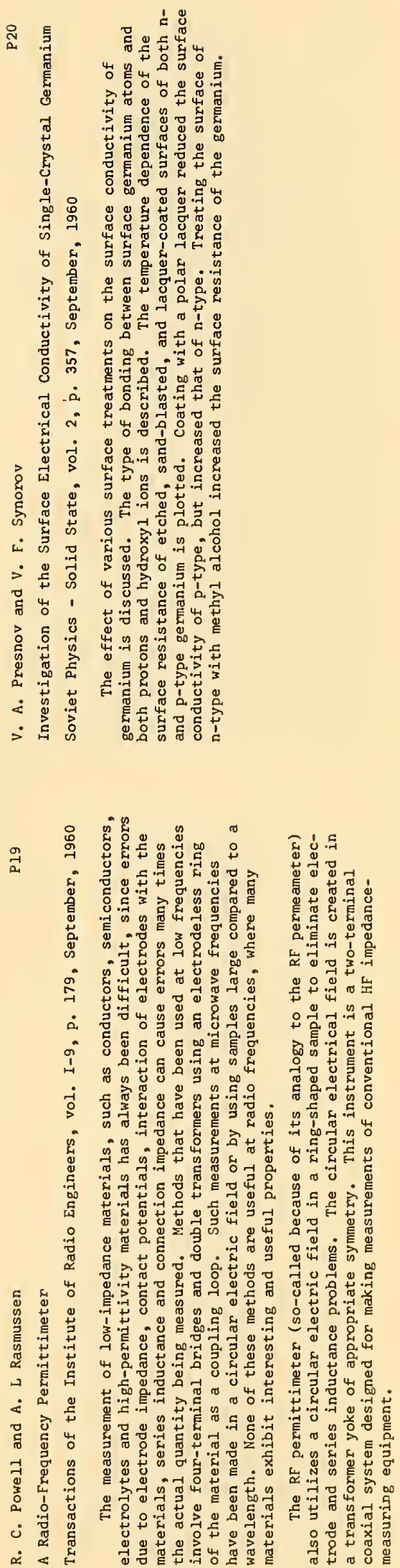

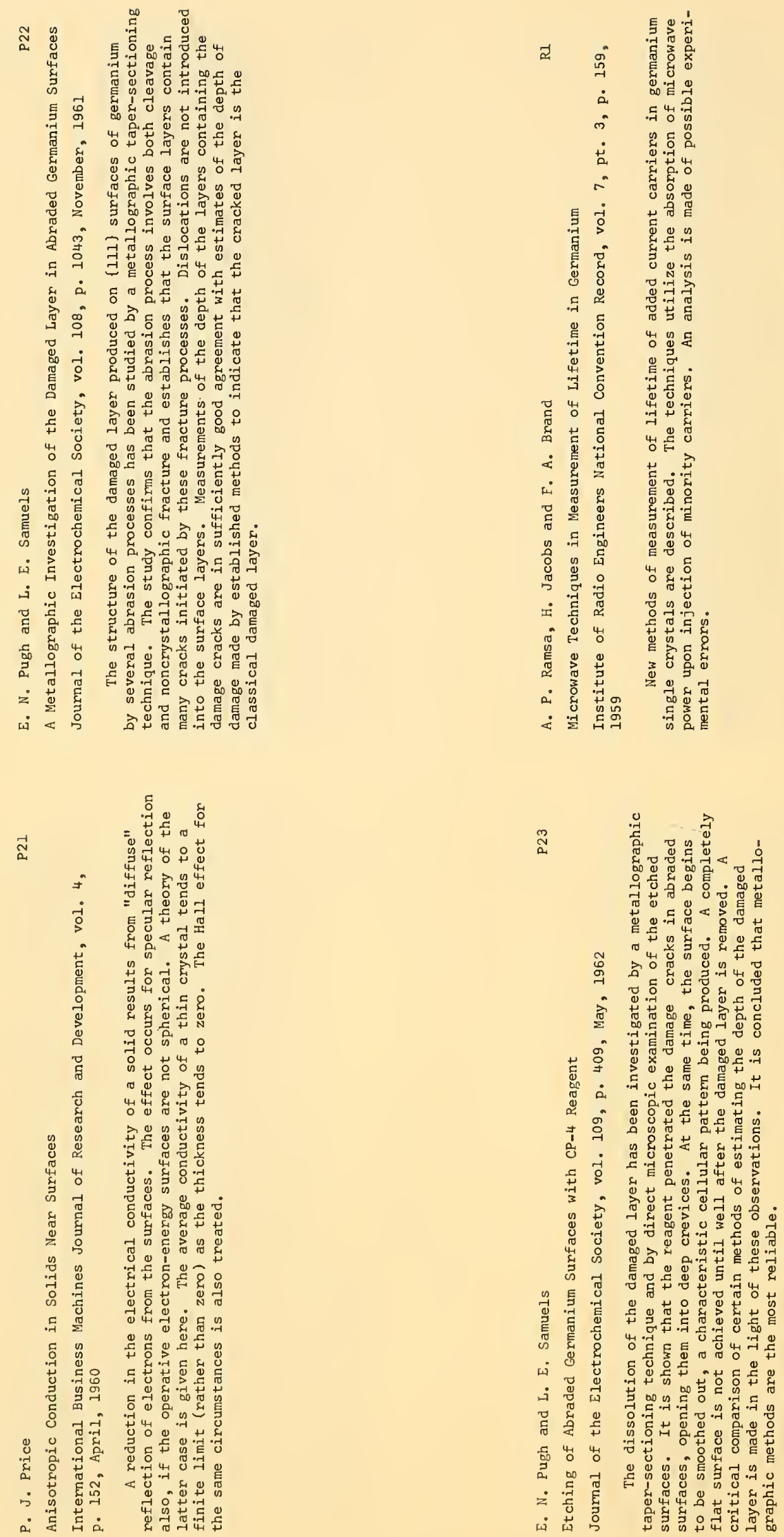

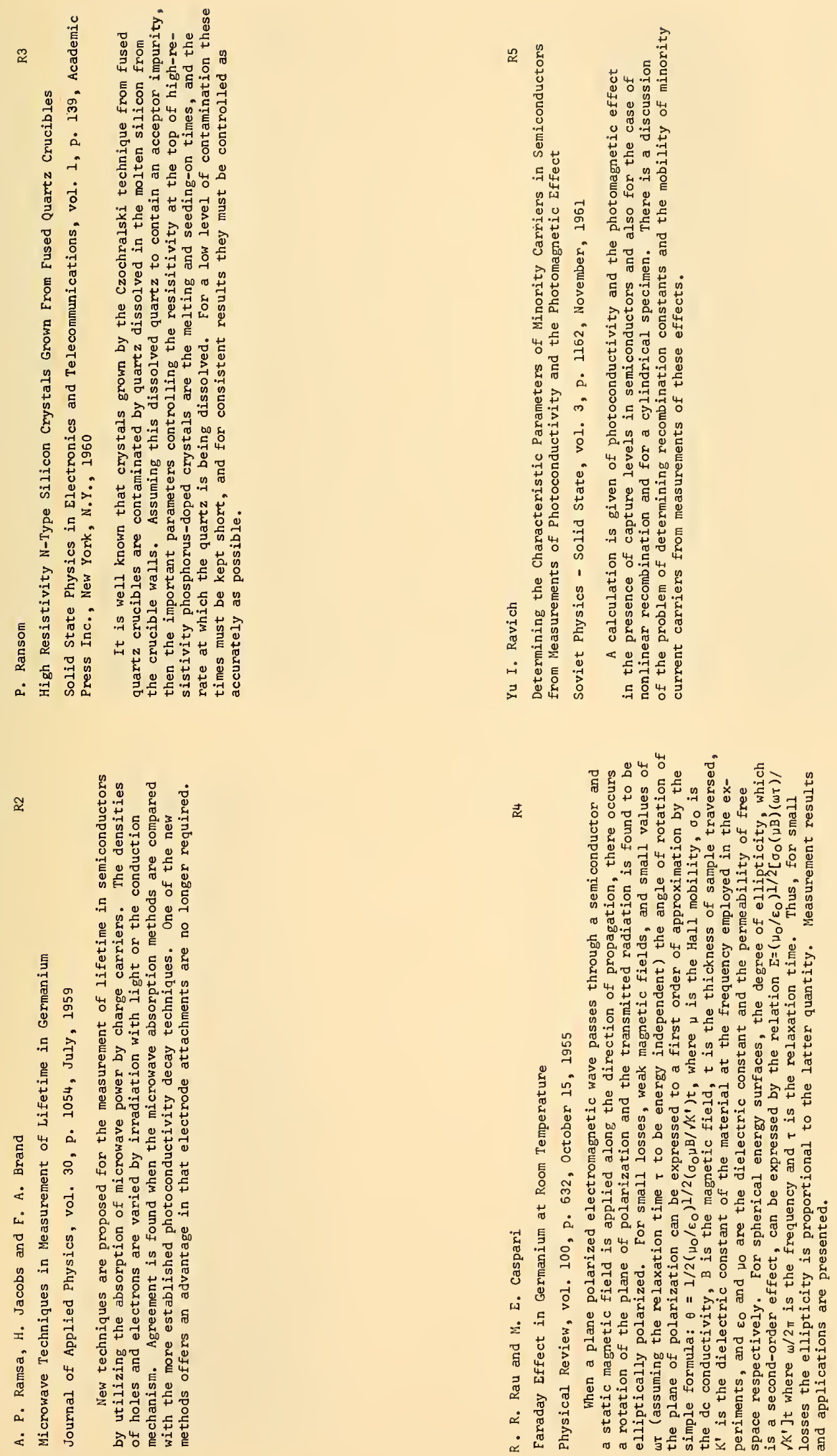

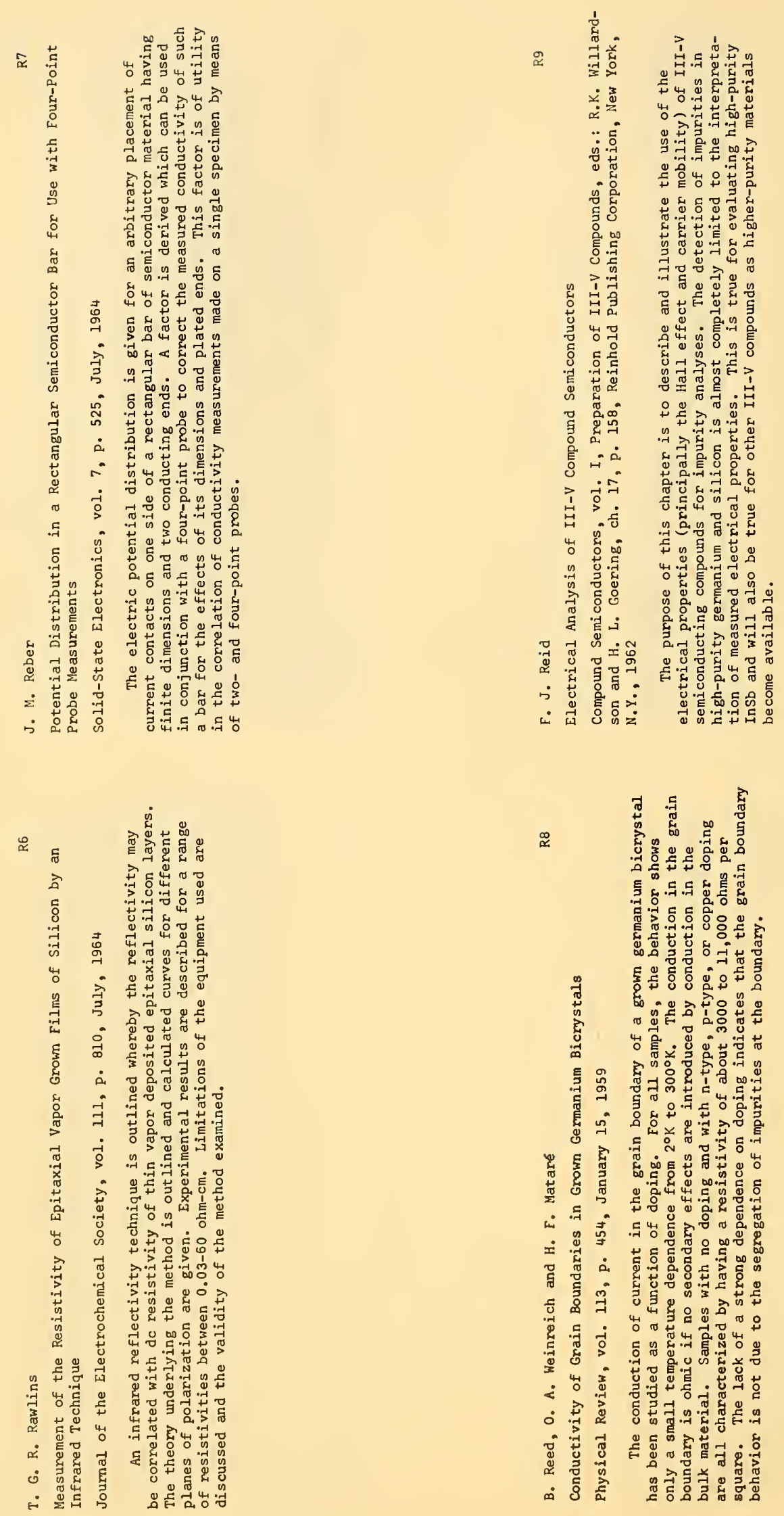


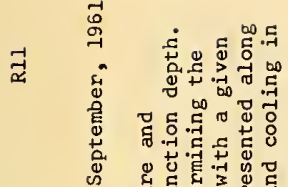

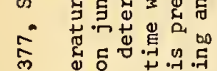

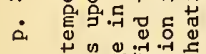

$$
\begin{aligned}
& \text { on } \\
& \text { 若 } \\
& \text { ᄃ } \\
& \text { 芴 }
\end{aligned}
$$

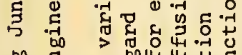

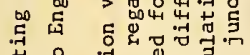

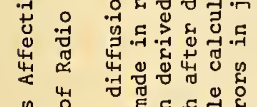

$$
\begin{aligned}
& \text { 品 }
\end{aligned}
$$

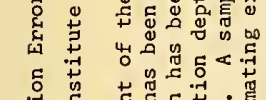

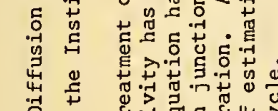

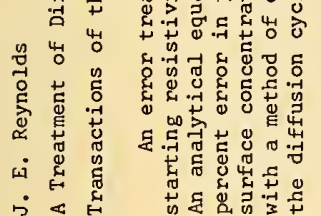

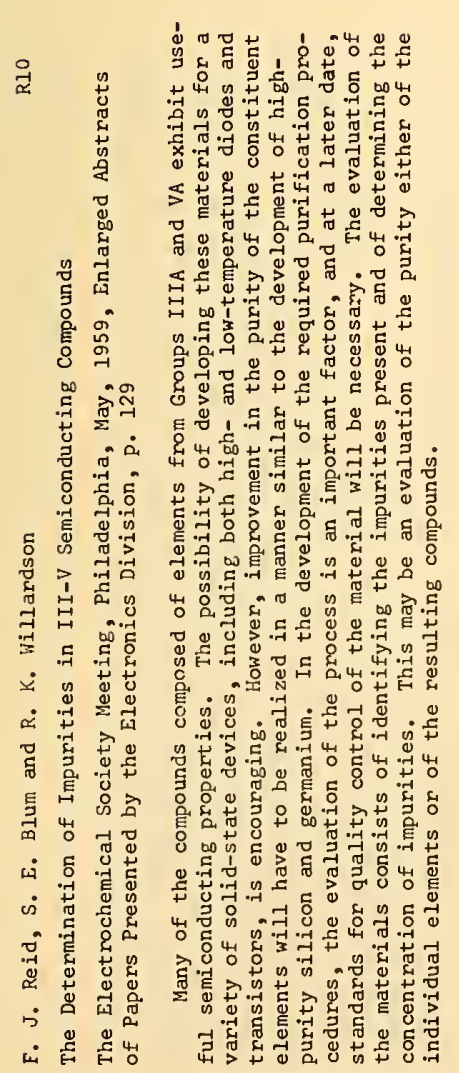

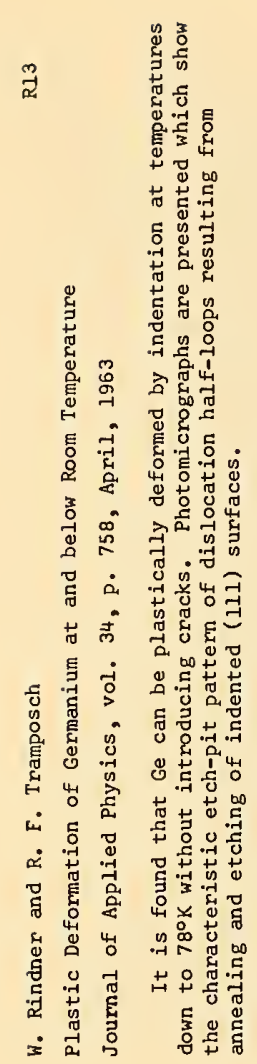

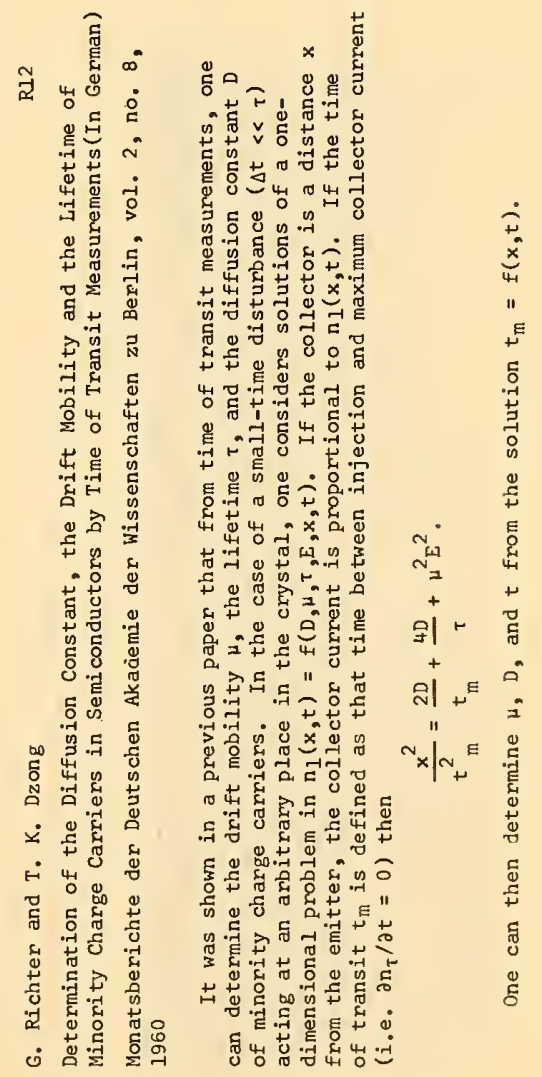



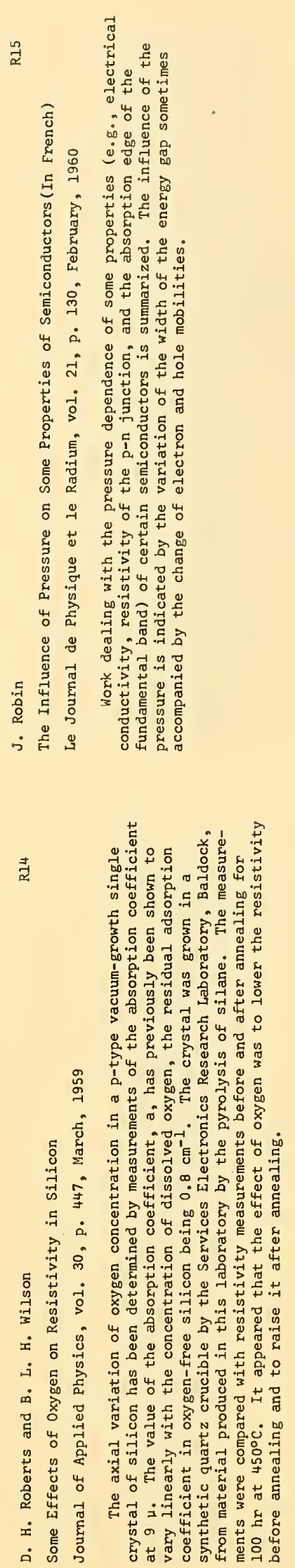
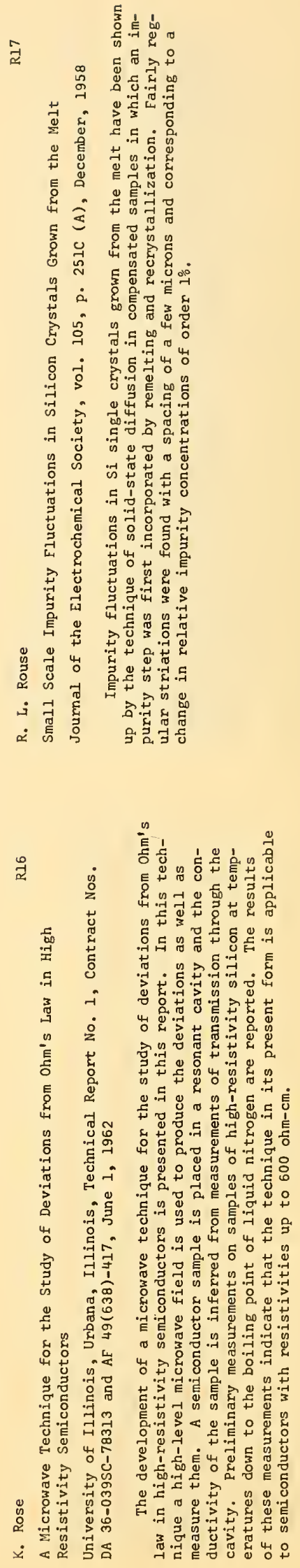

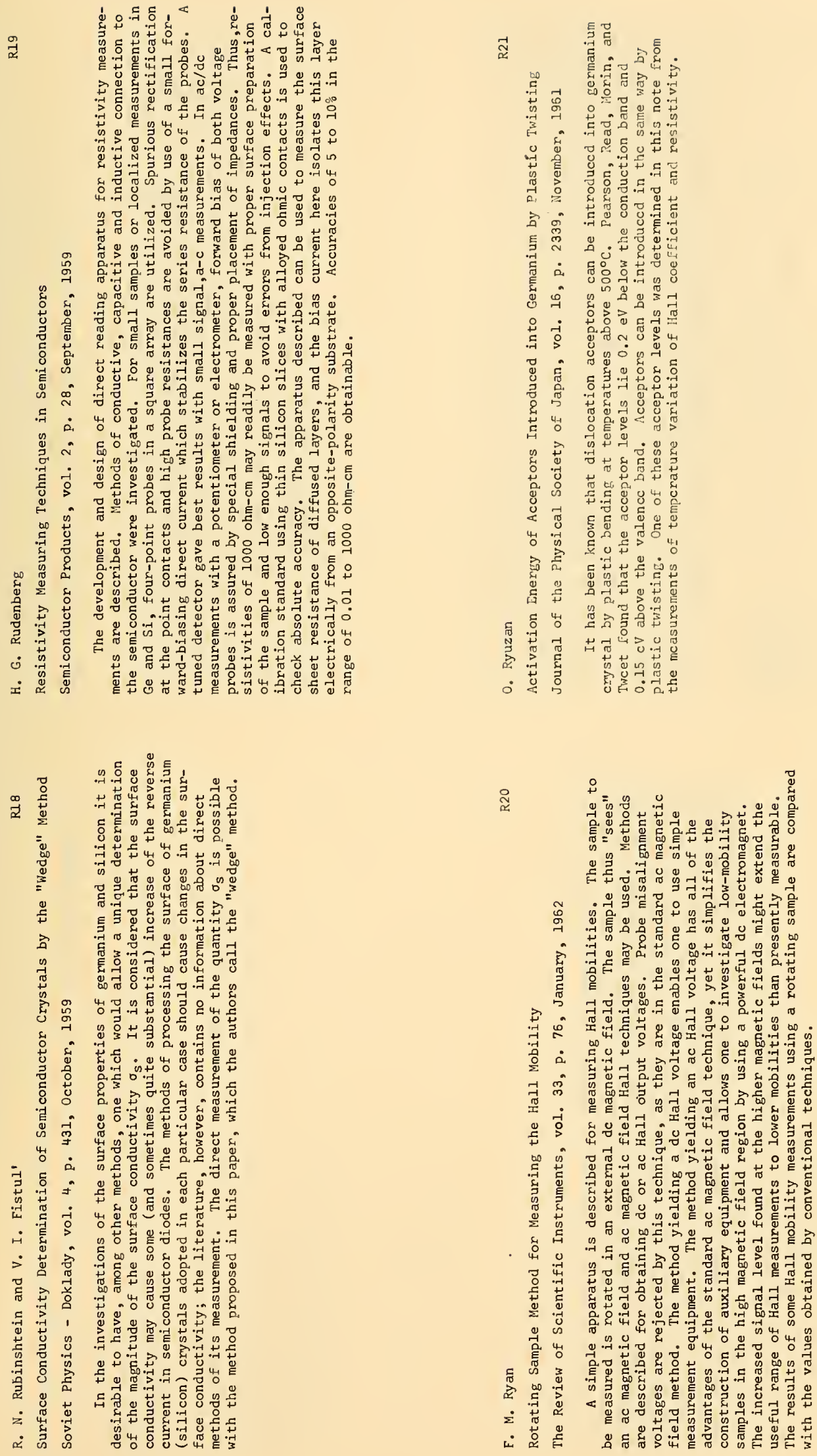

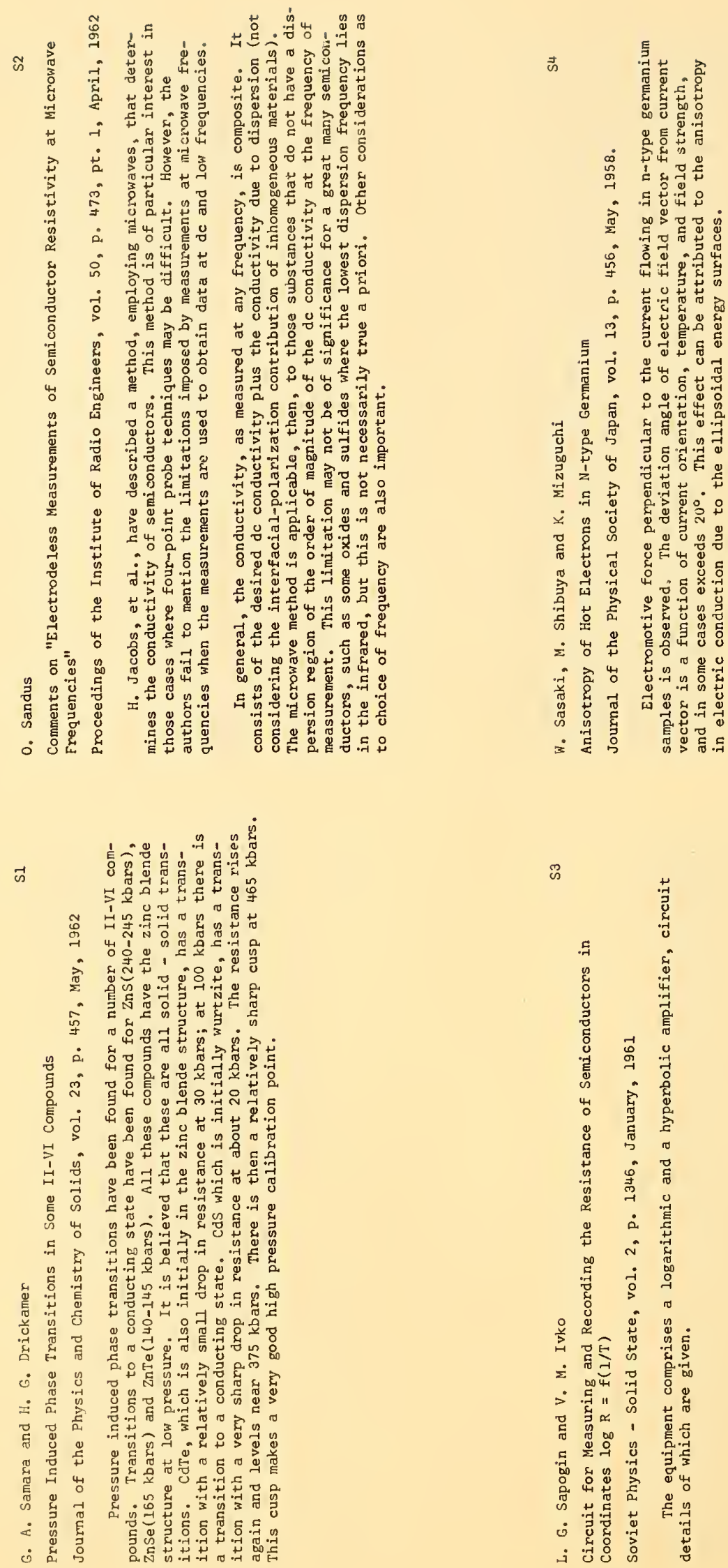

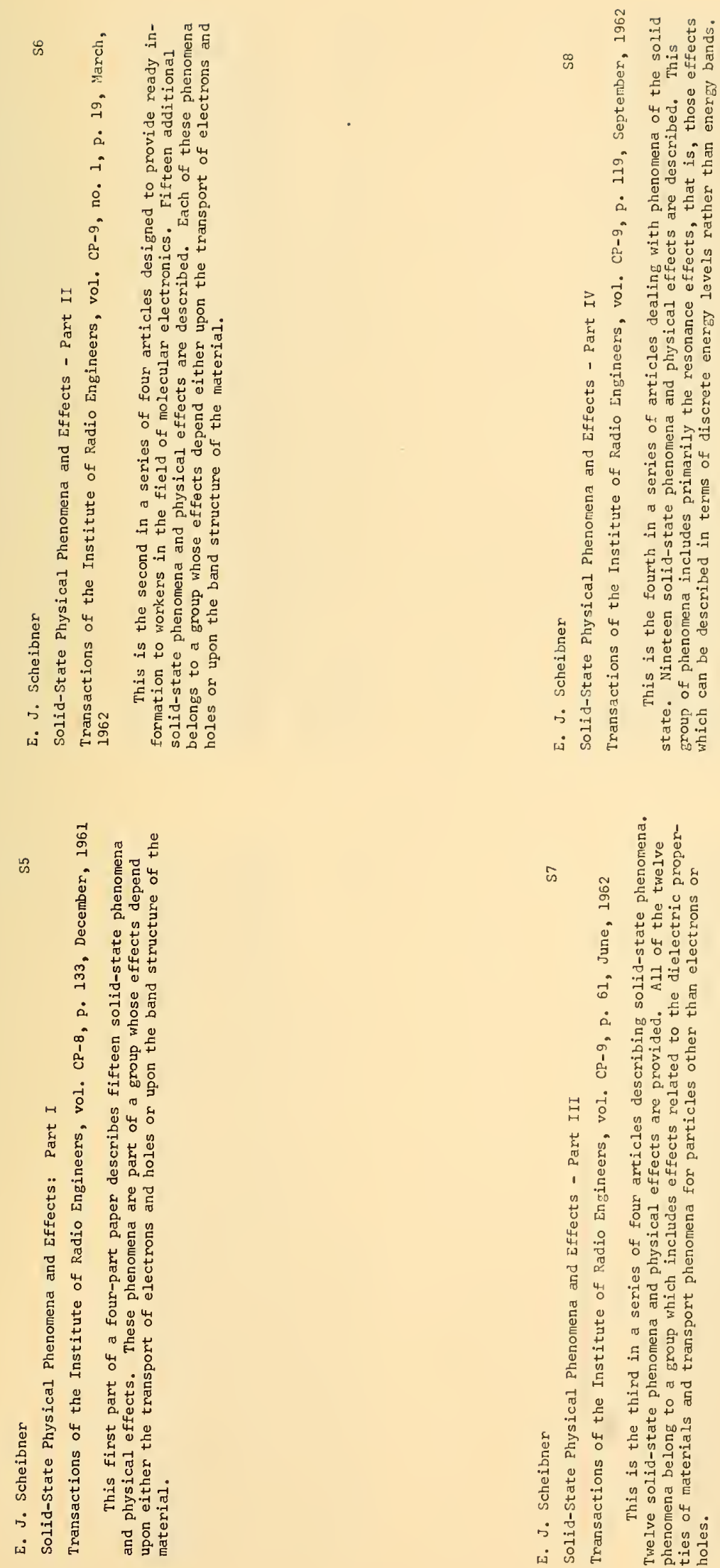

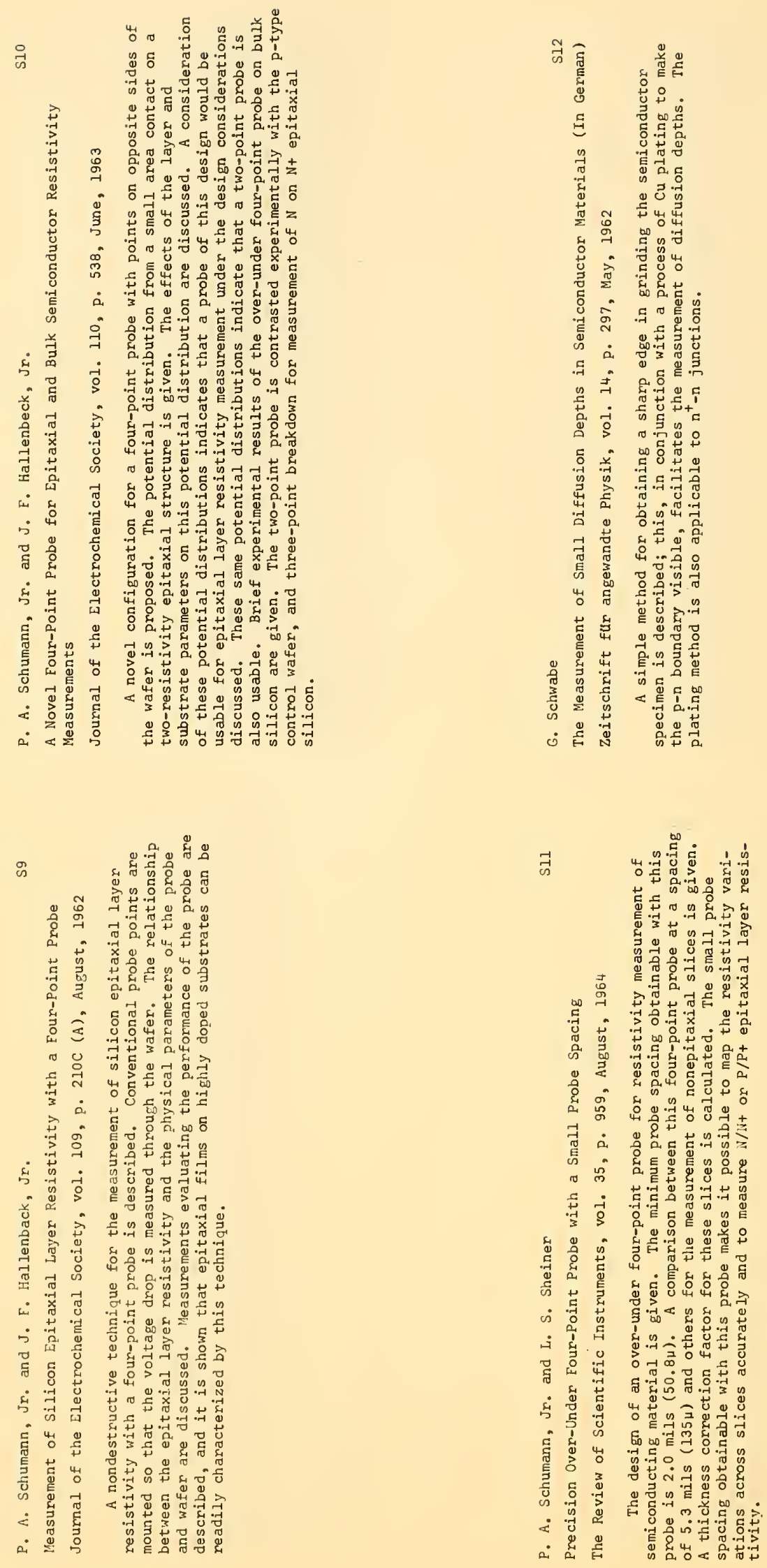


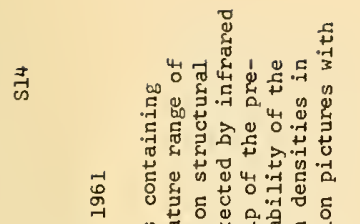

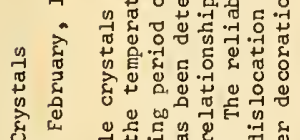

$$
\begin{aligned}
& \text { है } \\
& \text { 落 }
\end{aligned}
$$

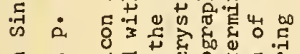

$$
\begin{aligned}
& \text { (5) }
\end{aligned}
$$

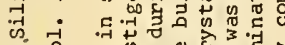

$$
\begin{aligned}
& \text {. }
\end{aligned}
$$

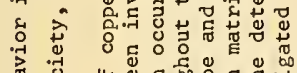

$$
\begin{aligned}
& \text { 贾 }
\end{aligned}
$$

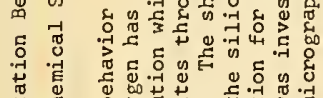

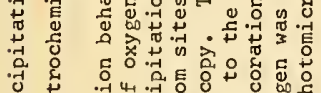

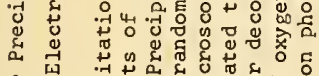

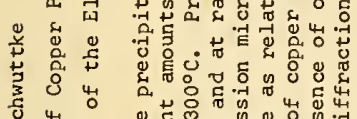

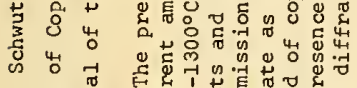

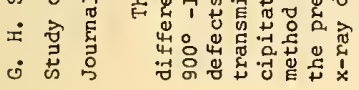
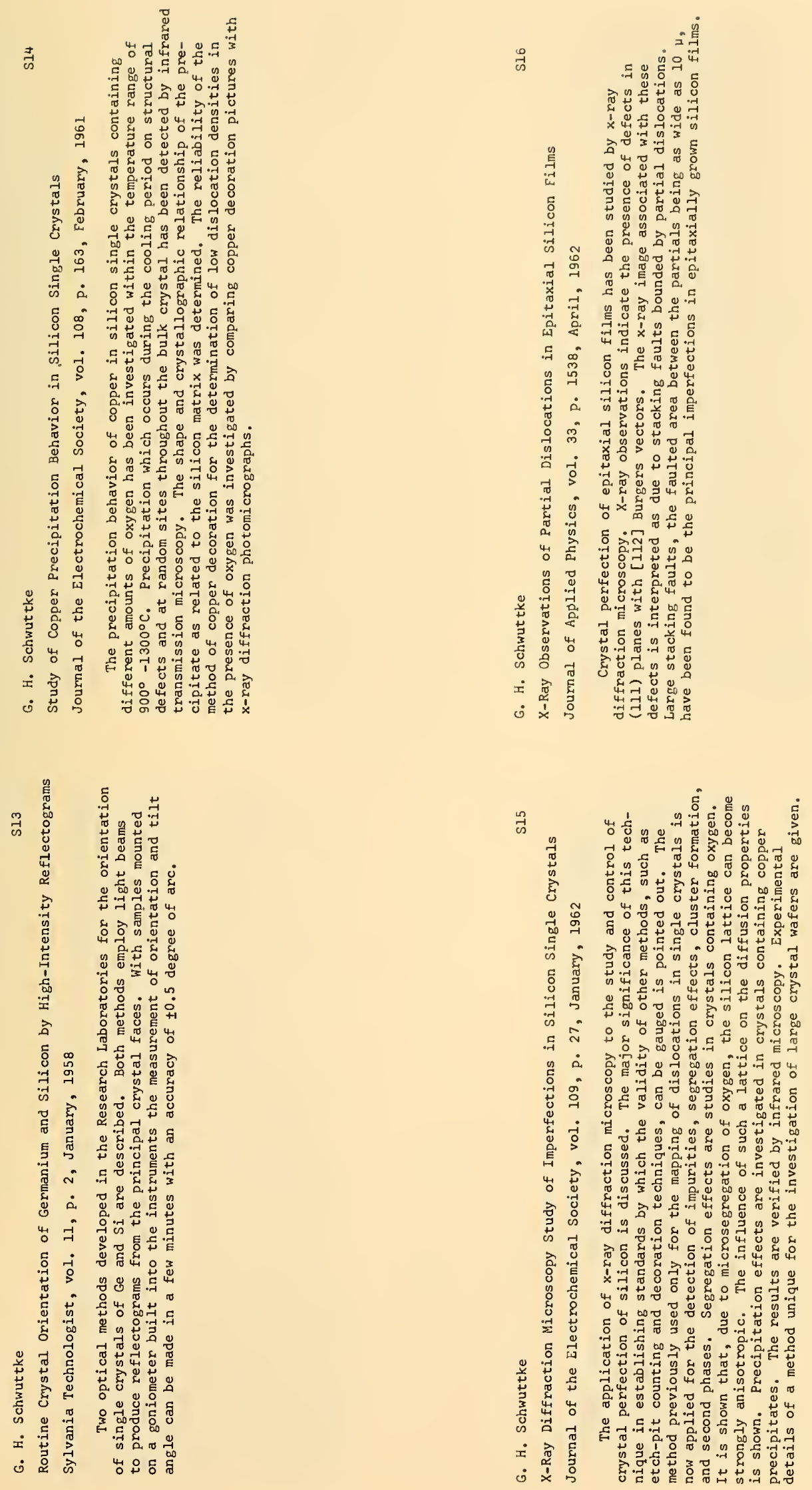

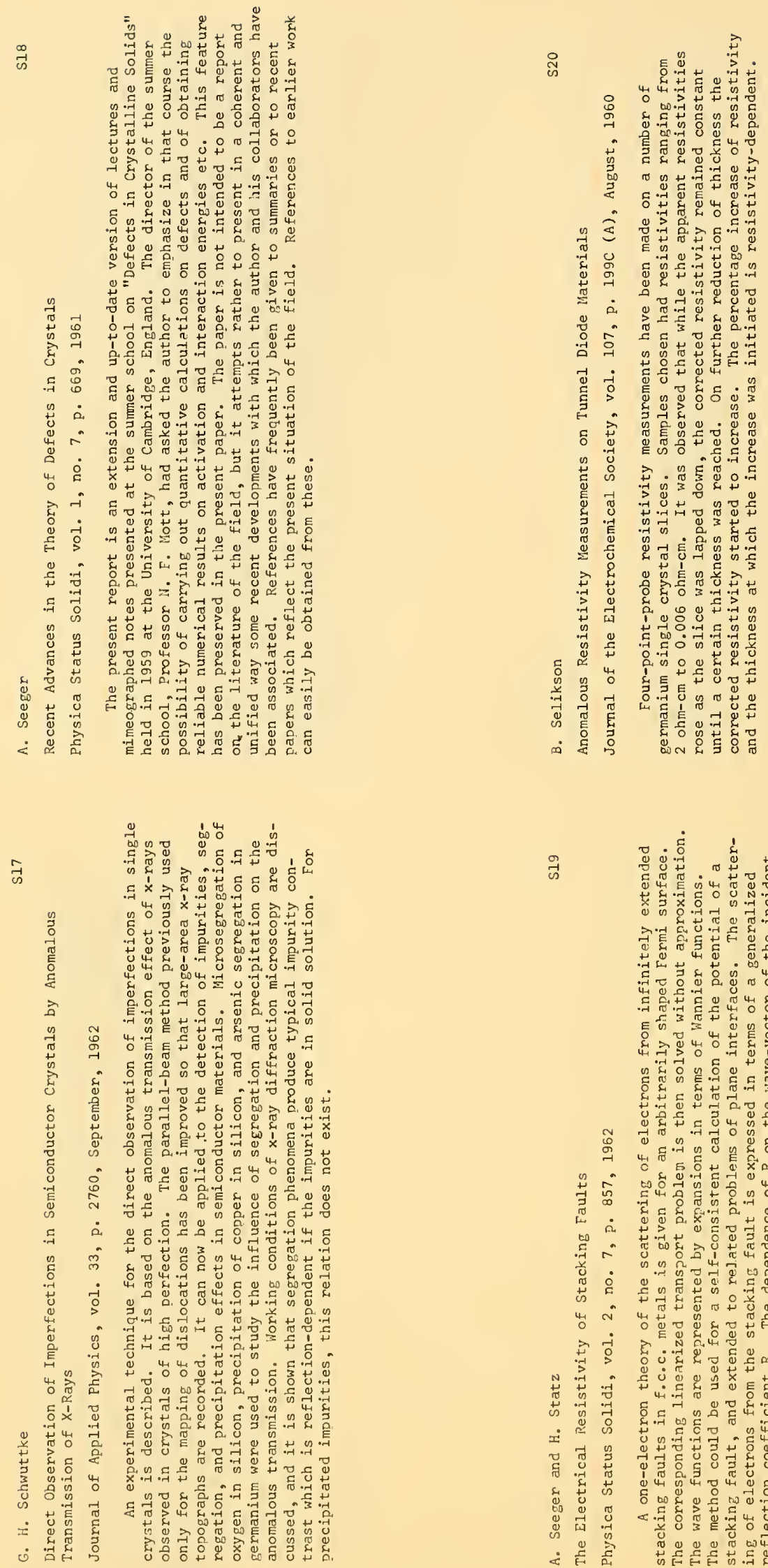

$\stackrel{9}{0}$

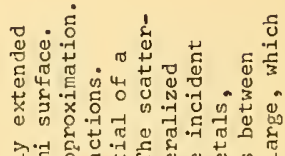

7.

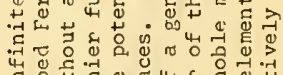

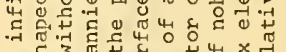

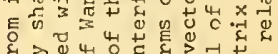

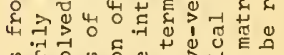

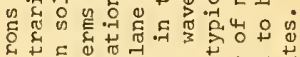

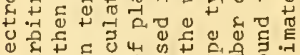

造 0

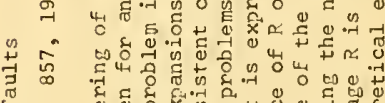

-

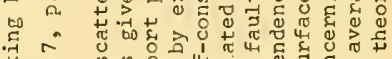

구의

4 过

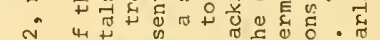

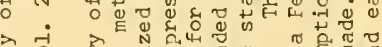

今.

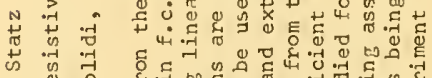

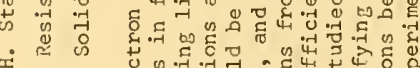

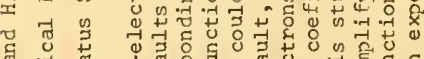

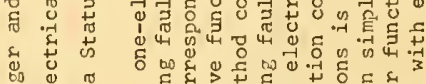

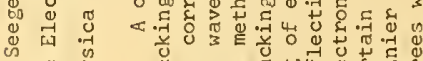

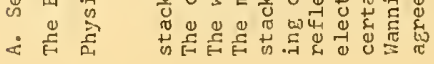



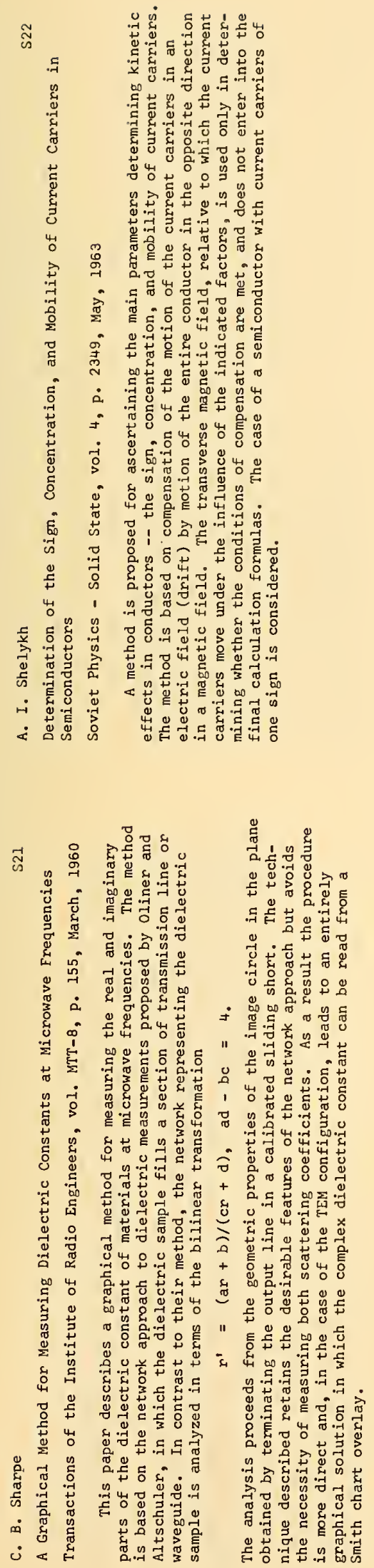
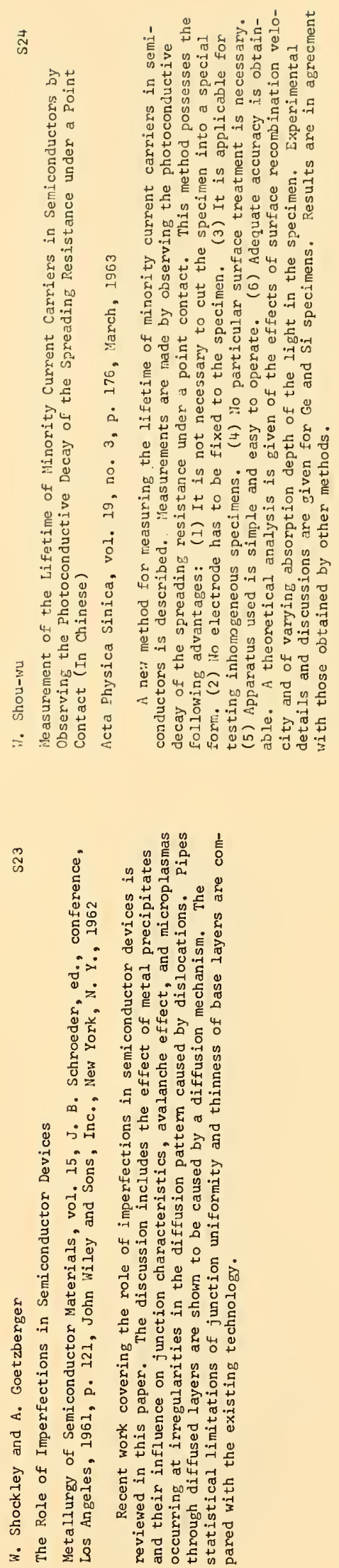

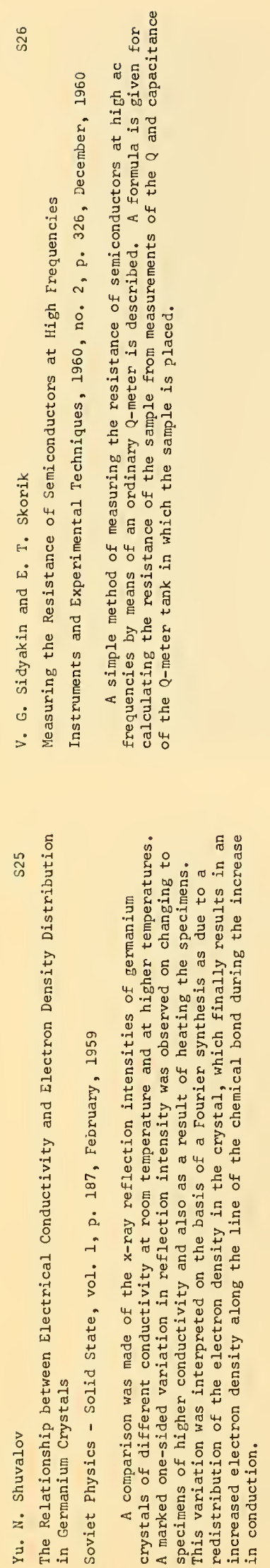
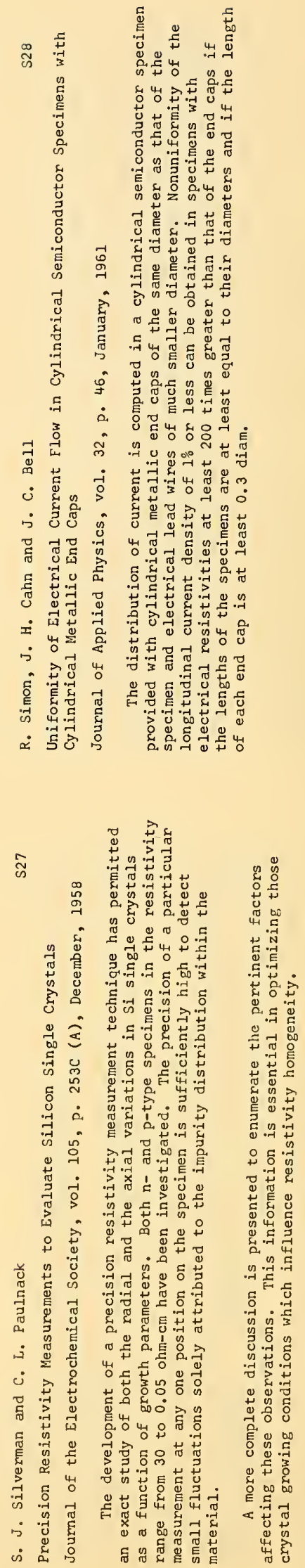

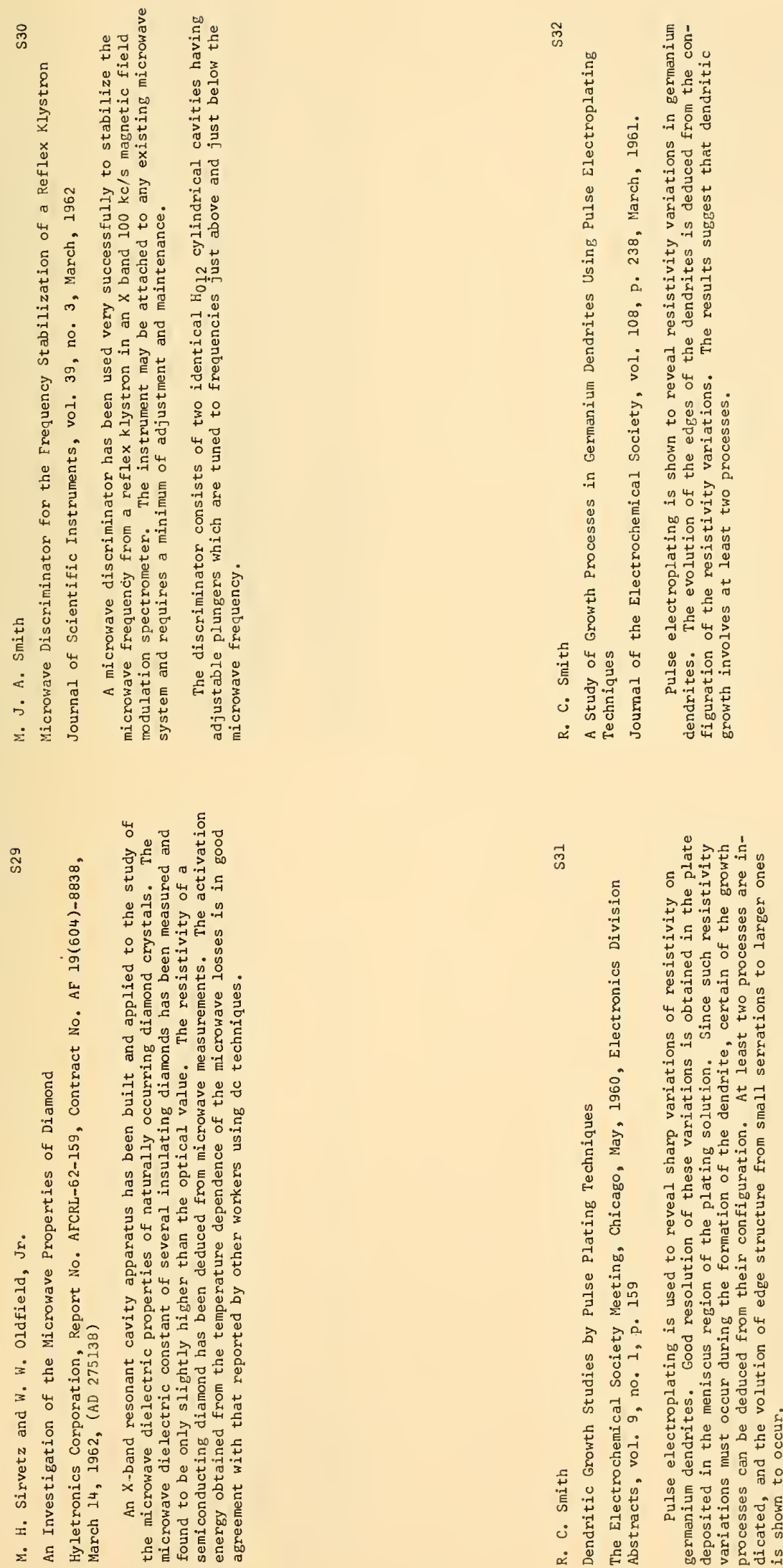

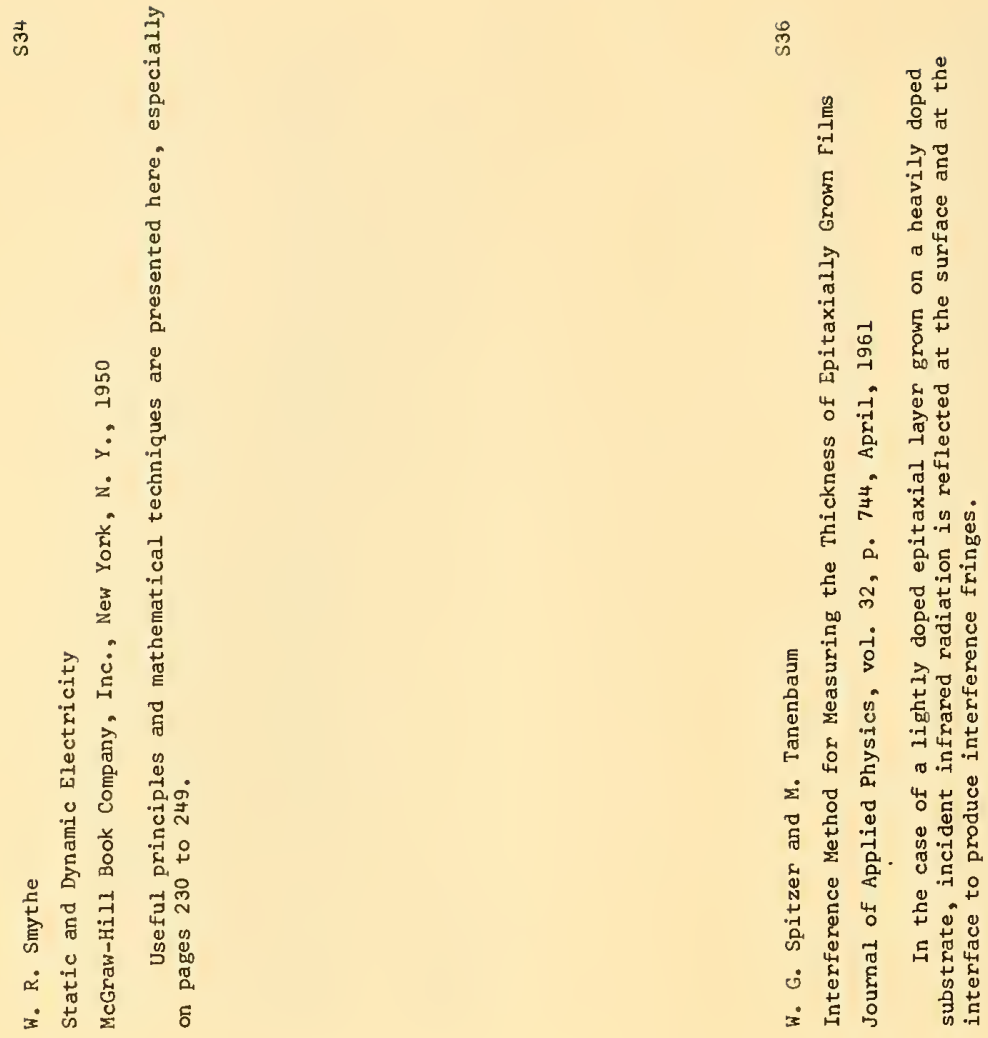

3.

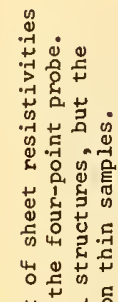

品

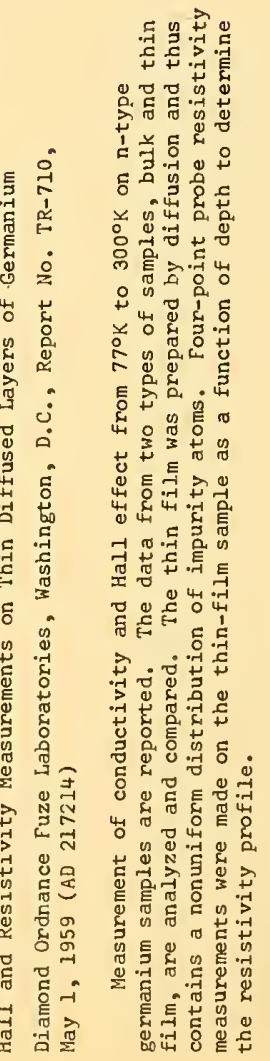



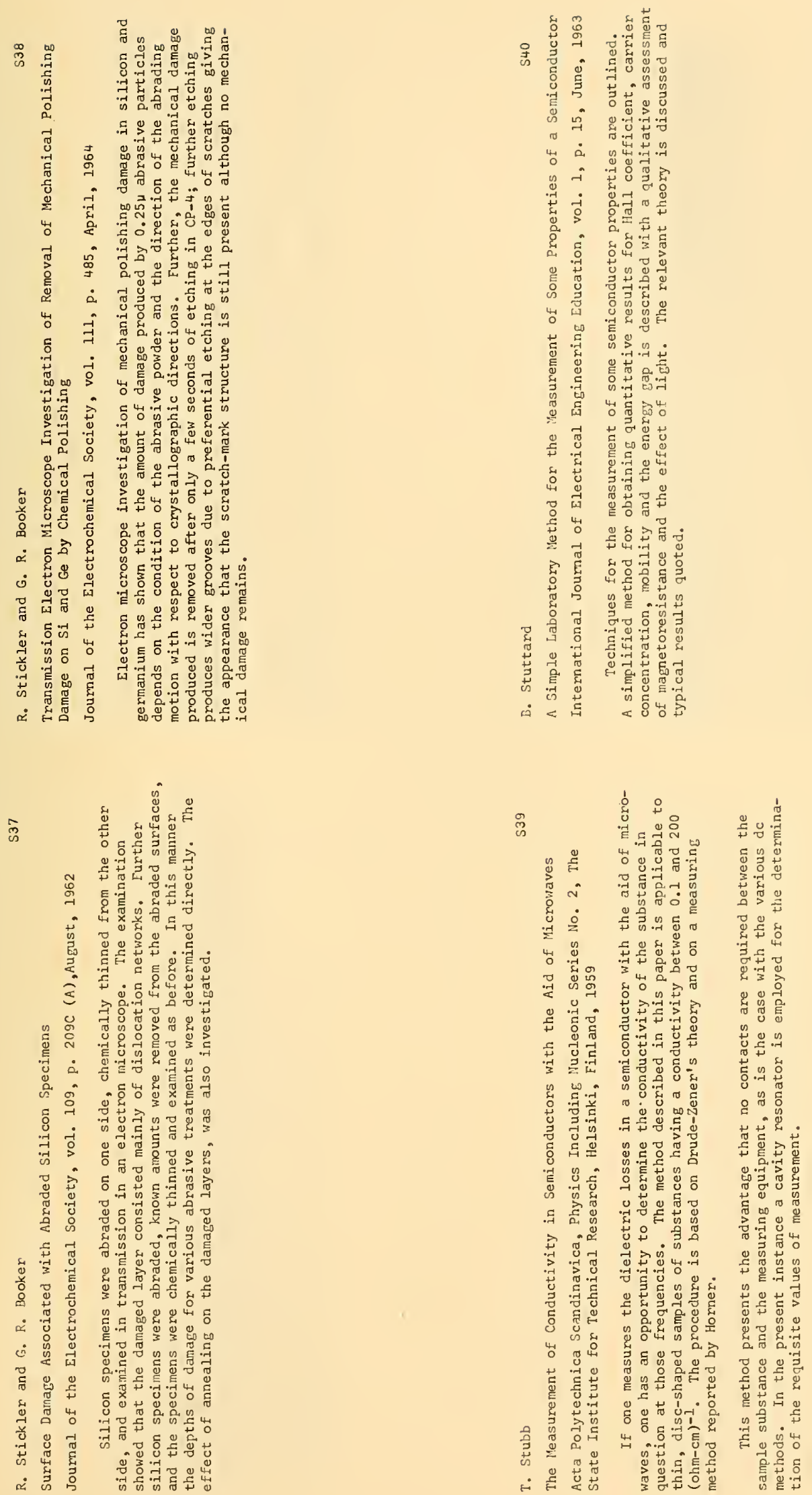

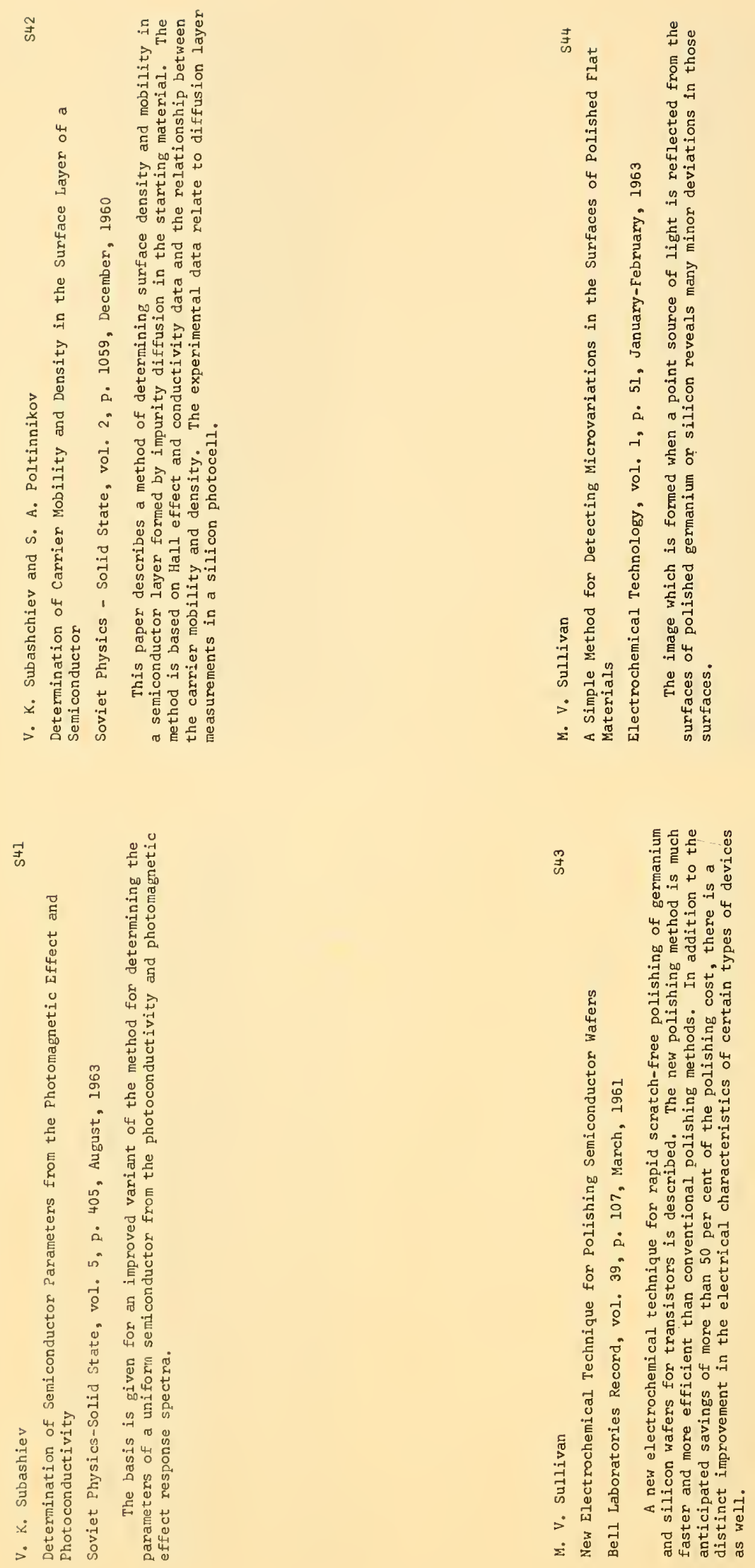

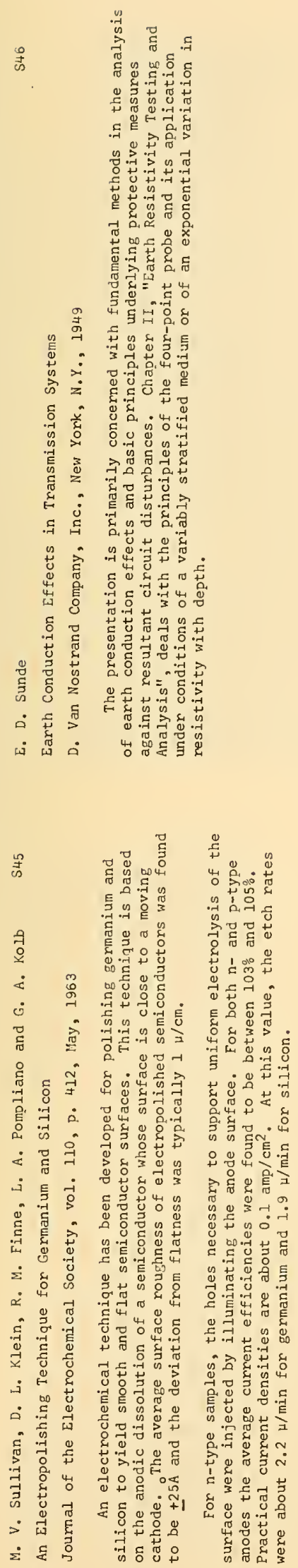
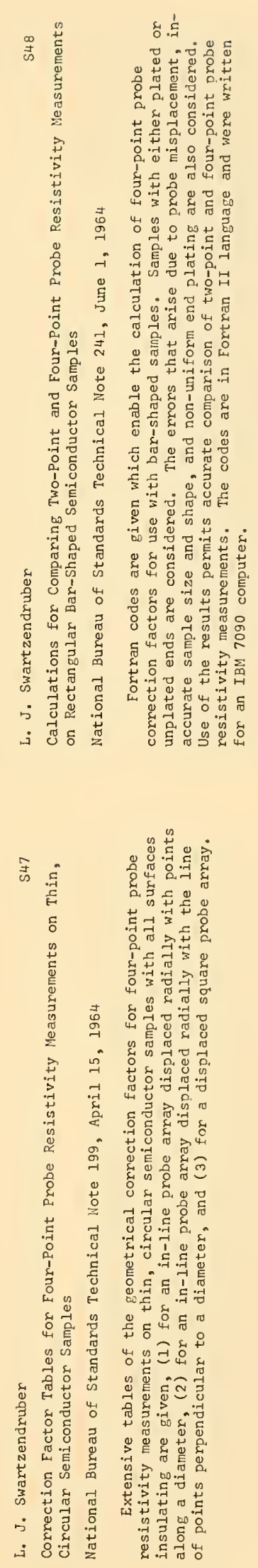

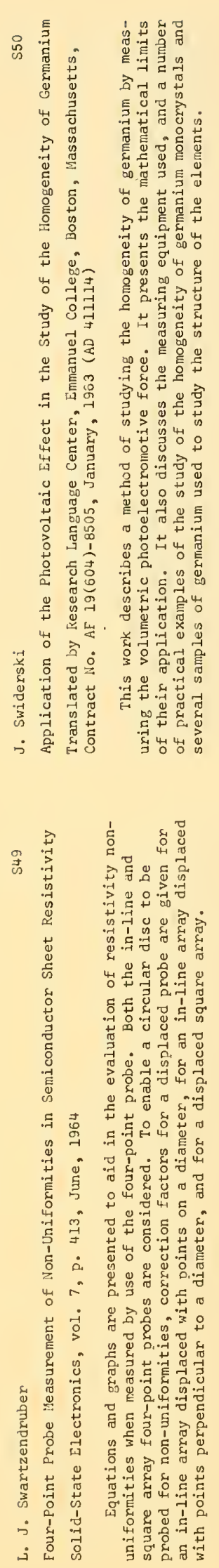
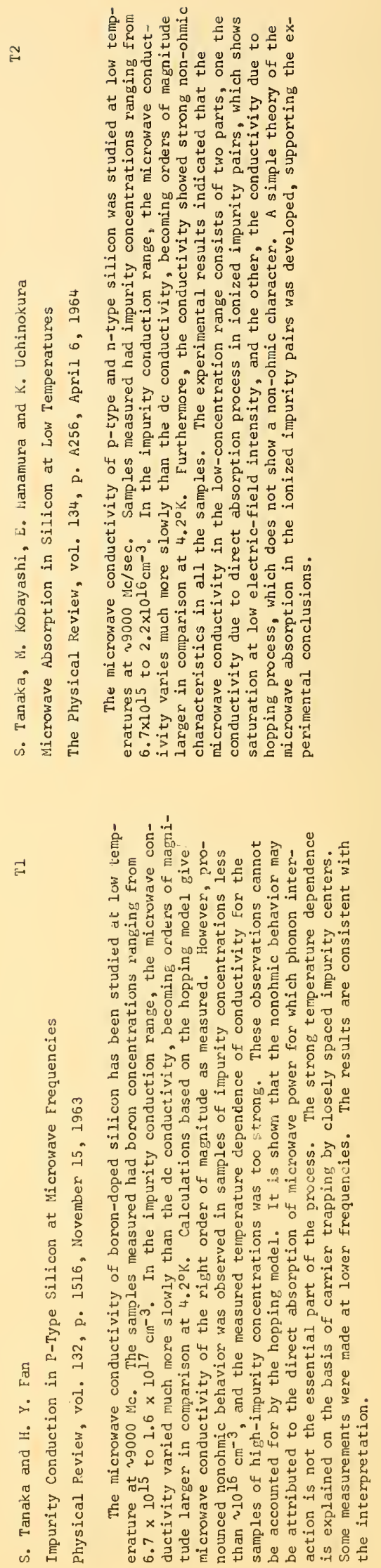

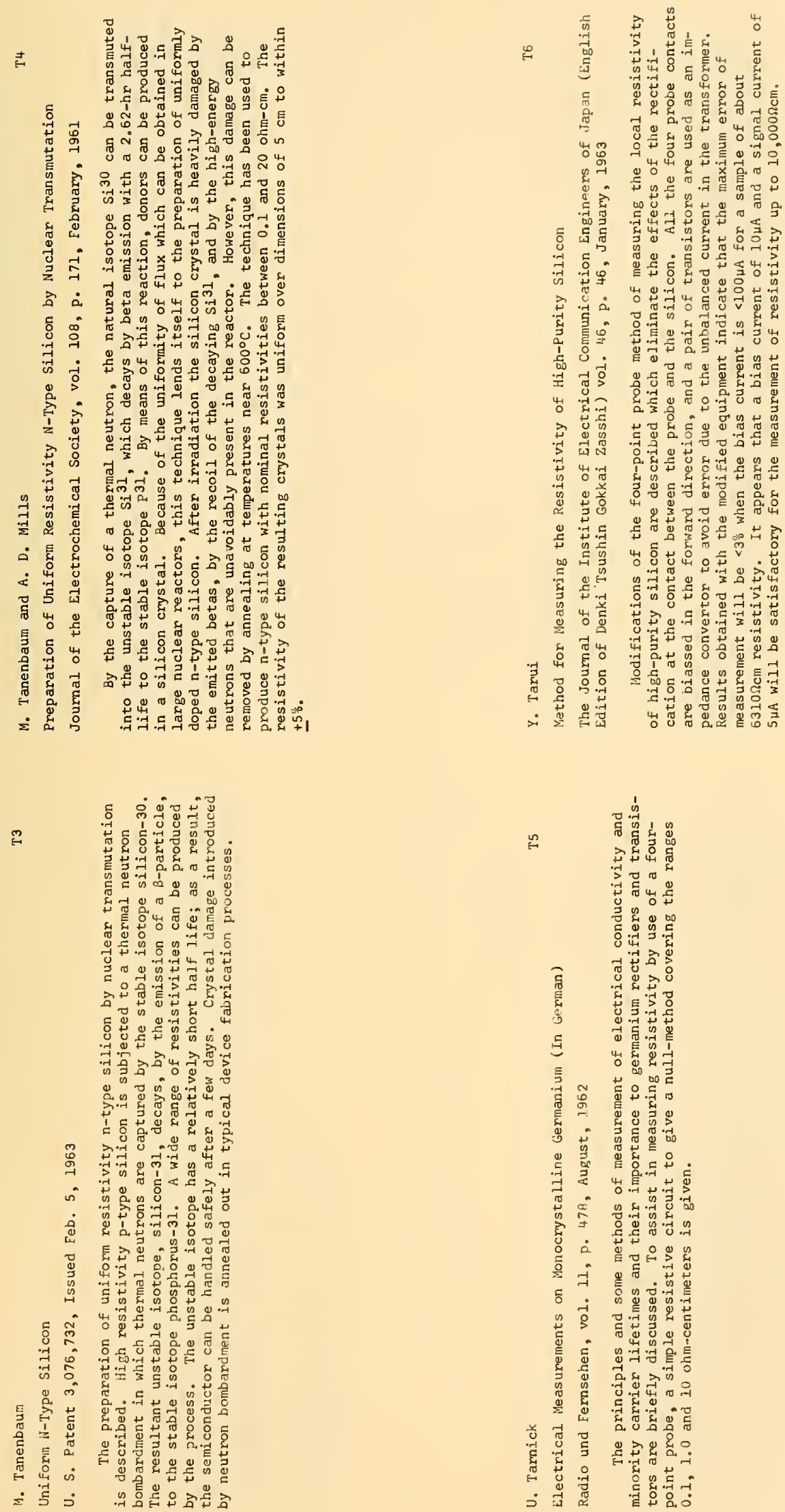


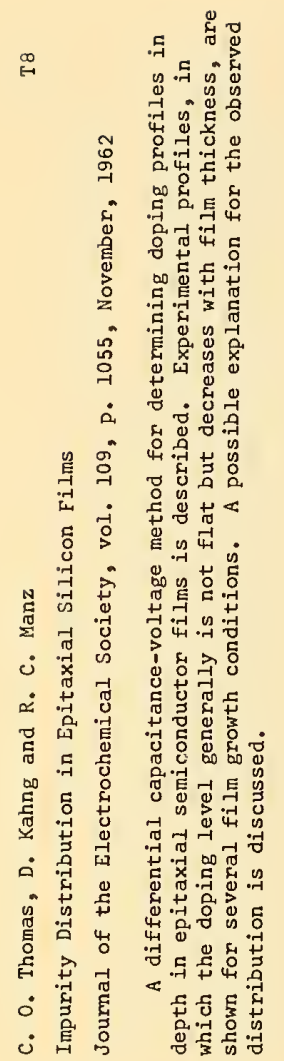

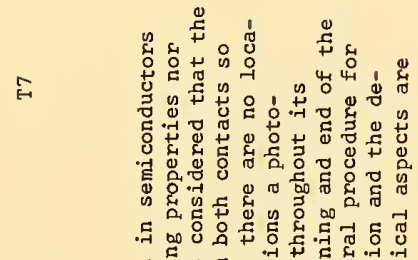

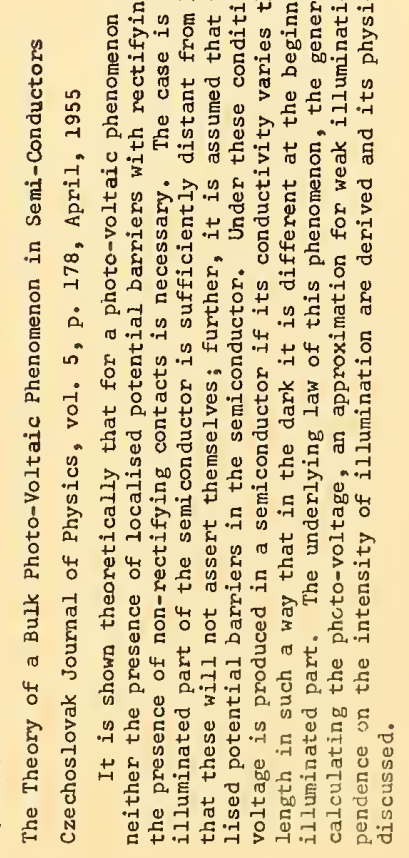

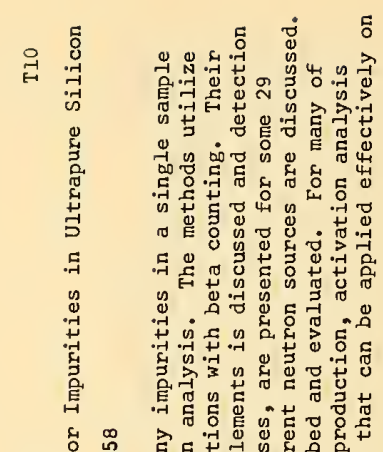
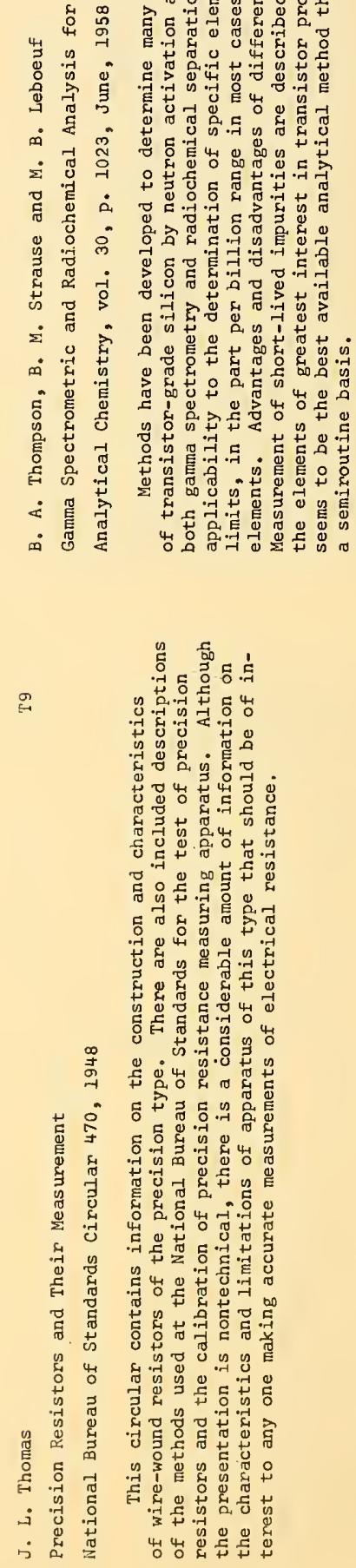

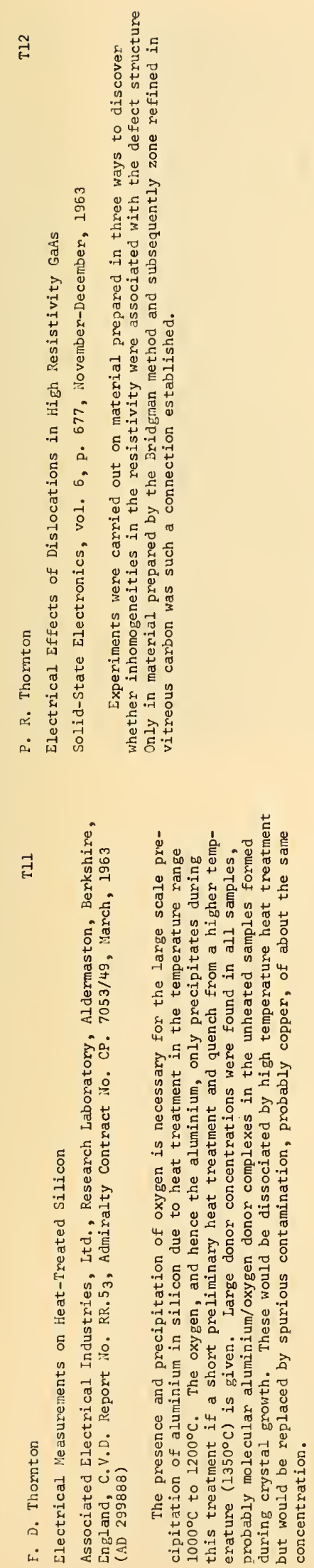
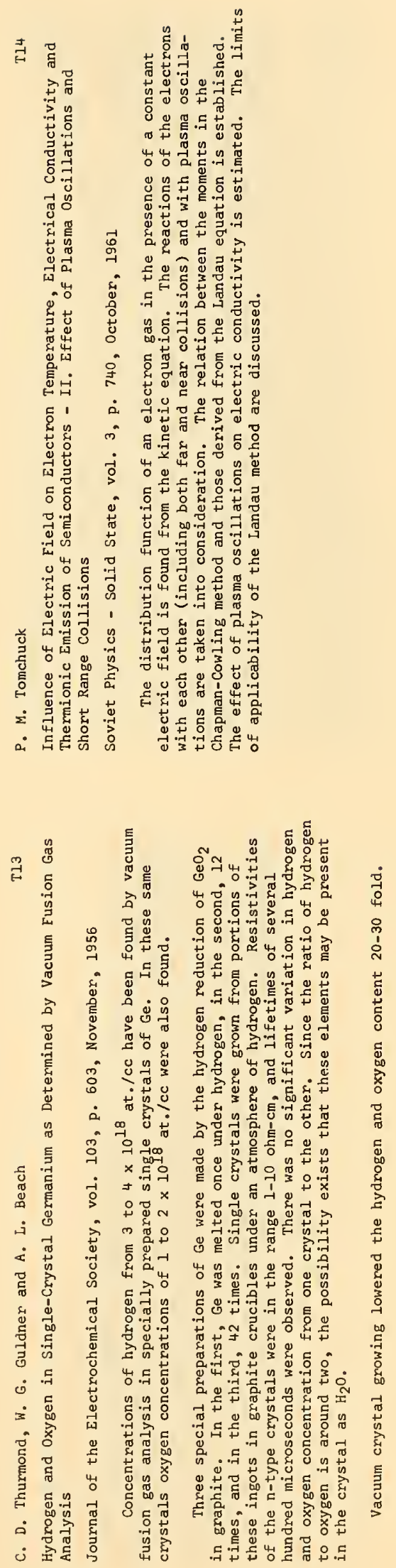

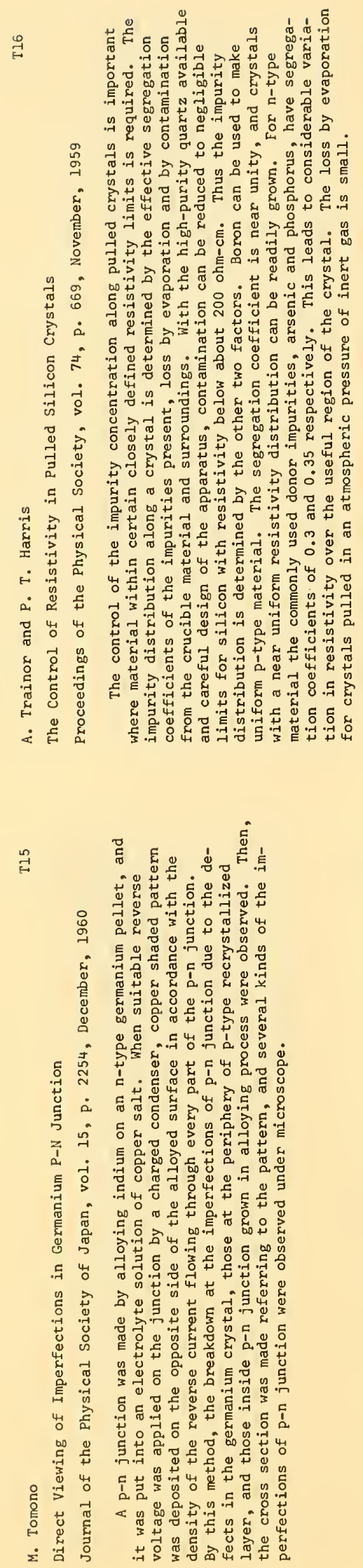
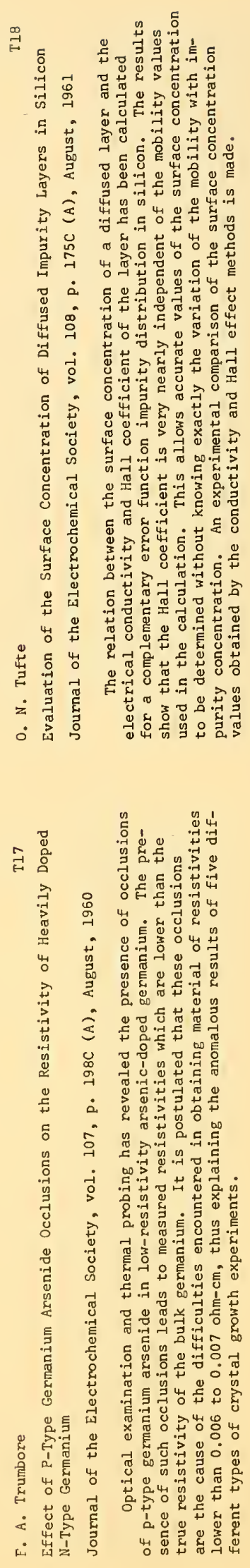


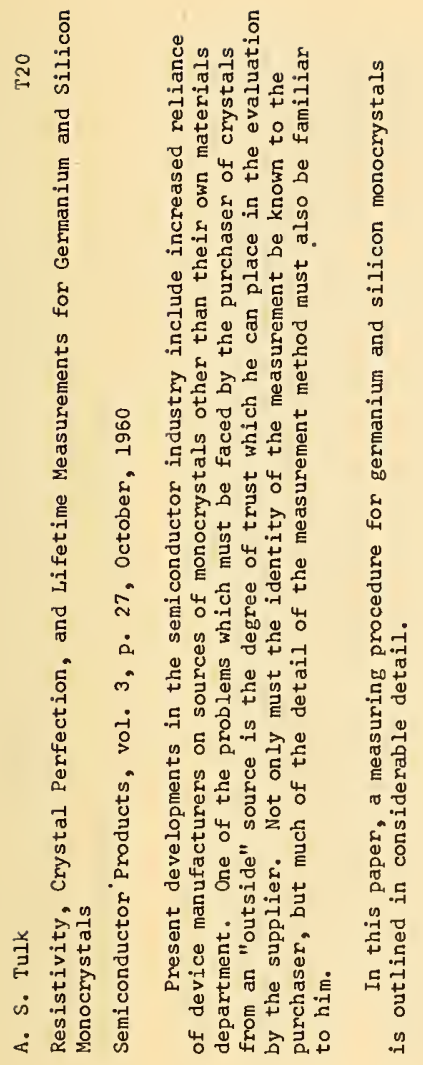

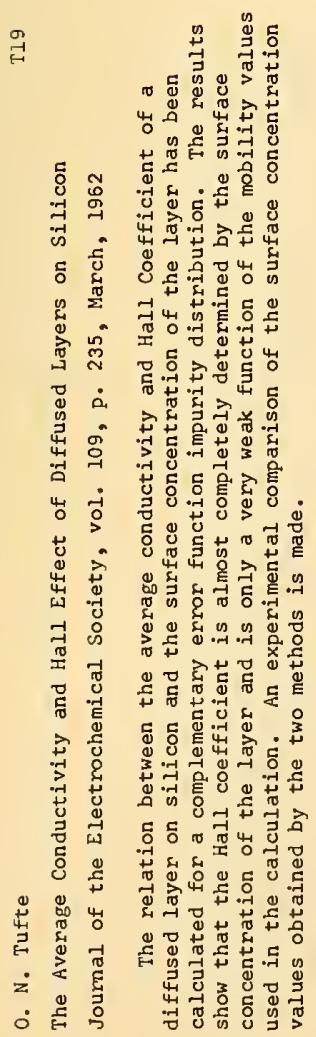

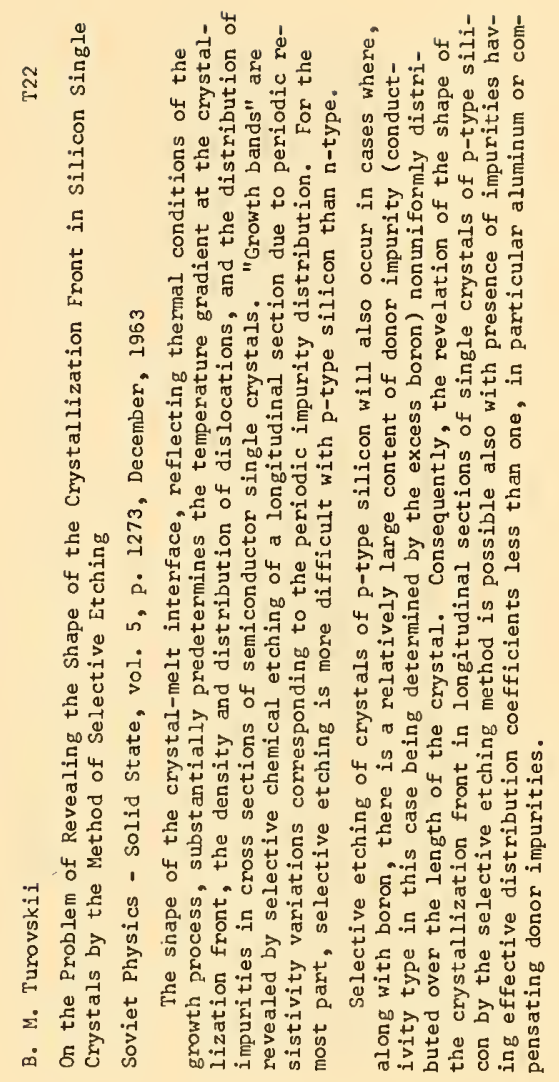

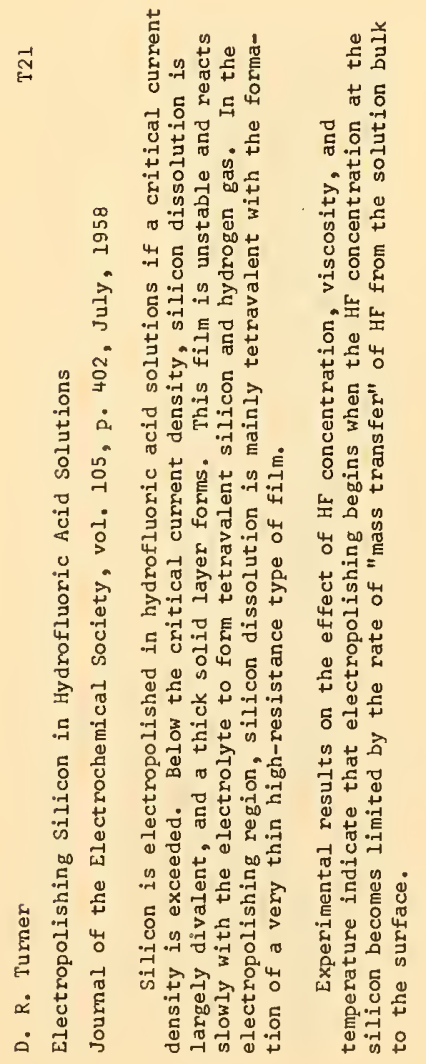



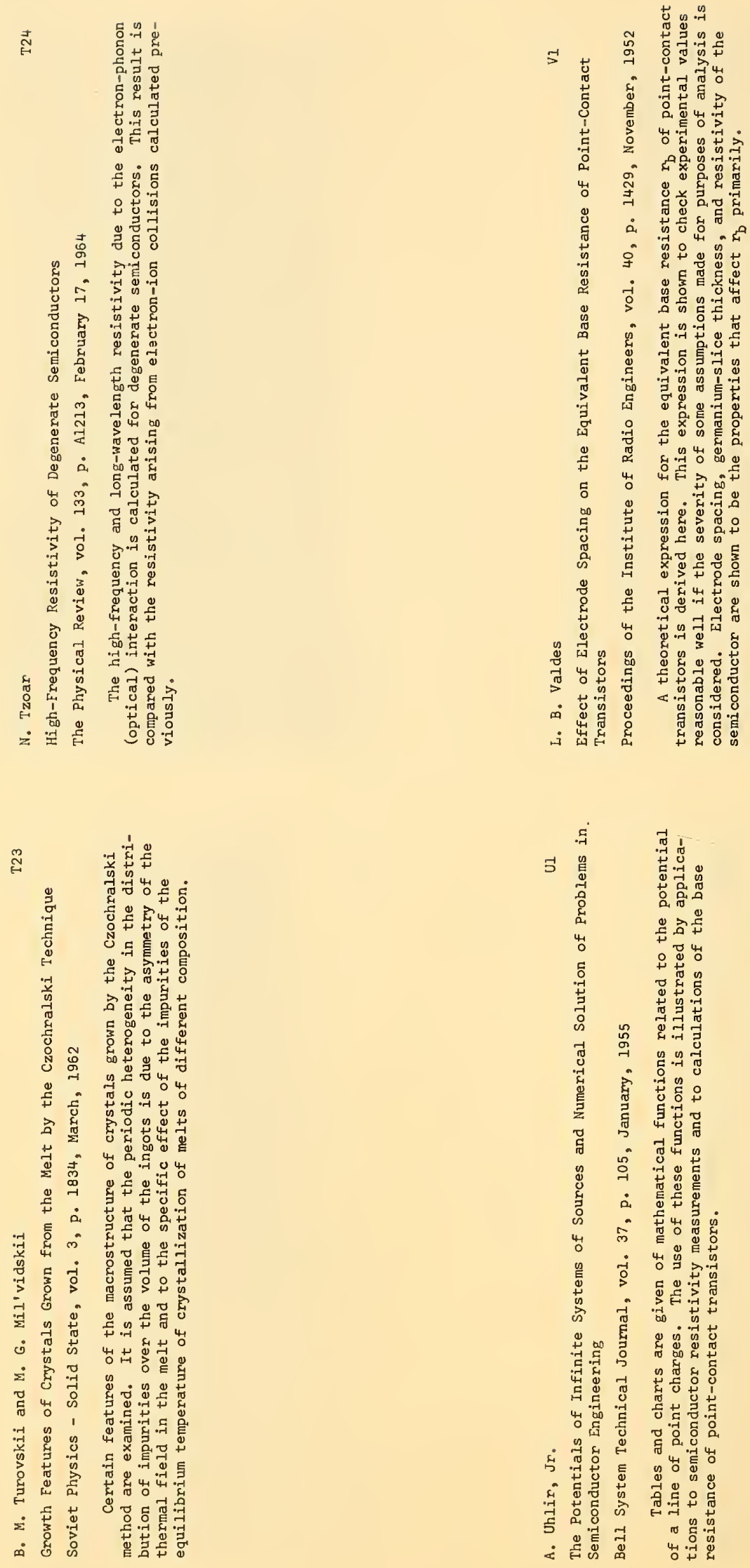


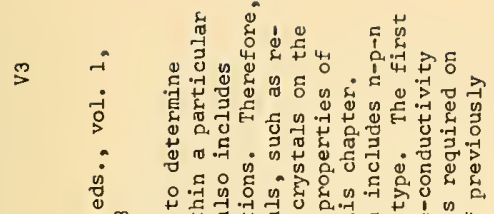

$$
\begin{aligned}
& \text { 舟 } \\
& \text { 势. } \\
& \text { की }
\end{aligned}
$$

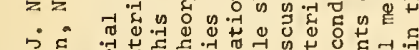

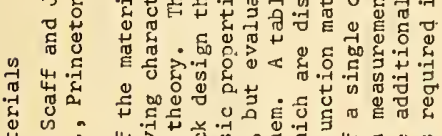

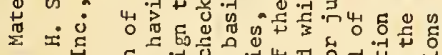

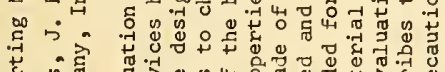

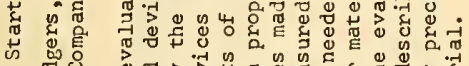

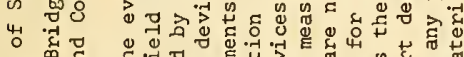

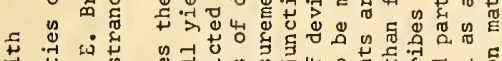

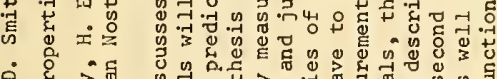

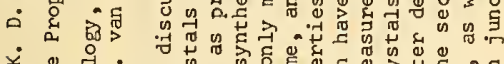

$$
\begin{aligned}
& \text { × }
\end{aligned}
$$

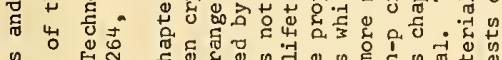

$$
\begin{aligned}
& \text { 品 }
\end{aligned}
$$

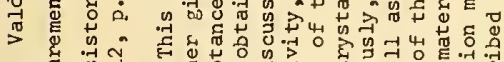

$$
\begin{aligned}
& \text { ๓ }
\end{aligned}
$$

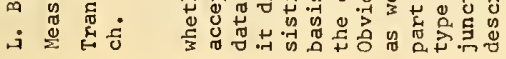
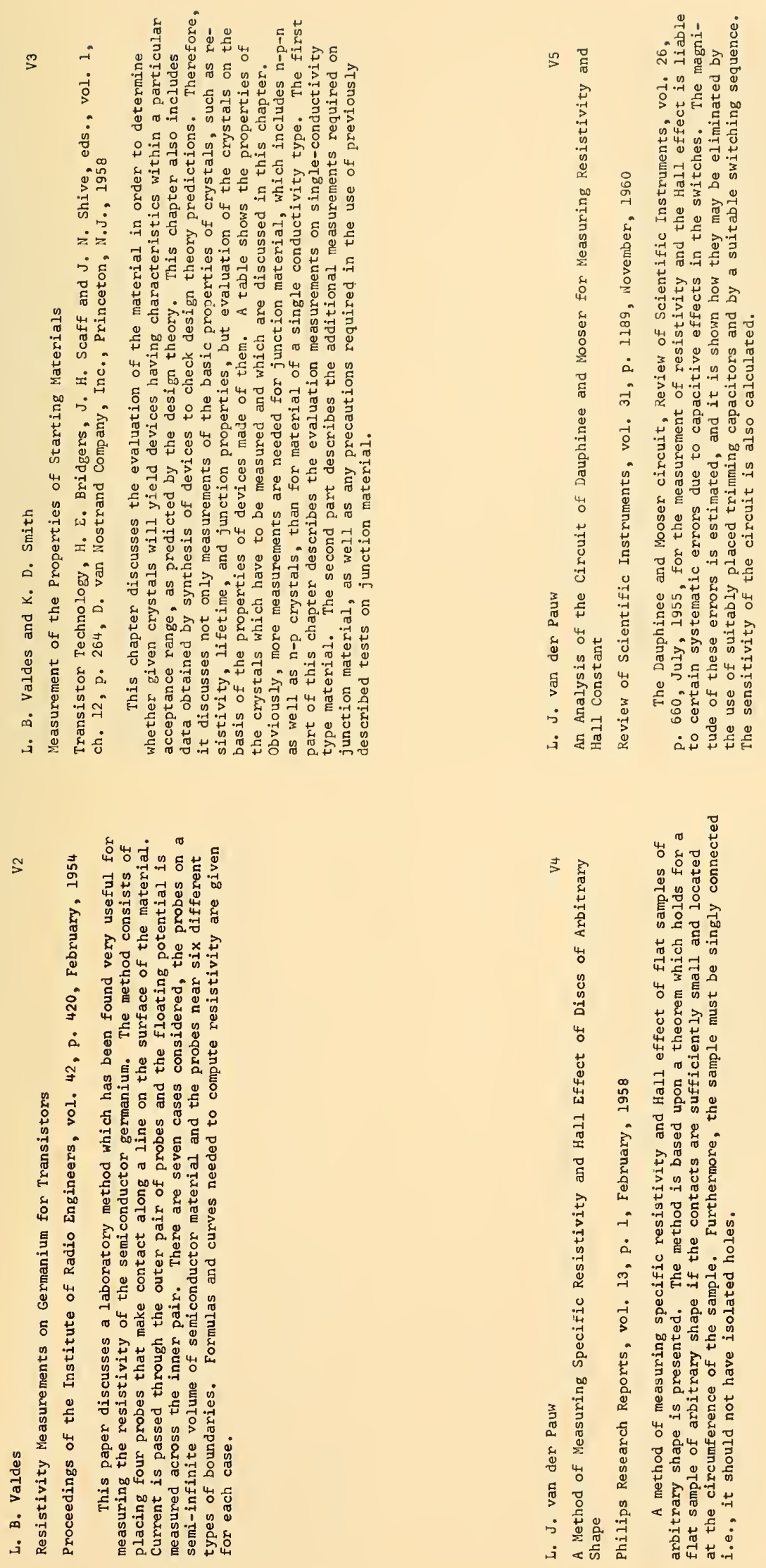

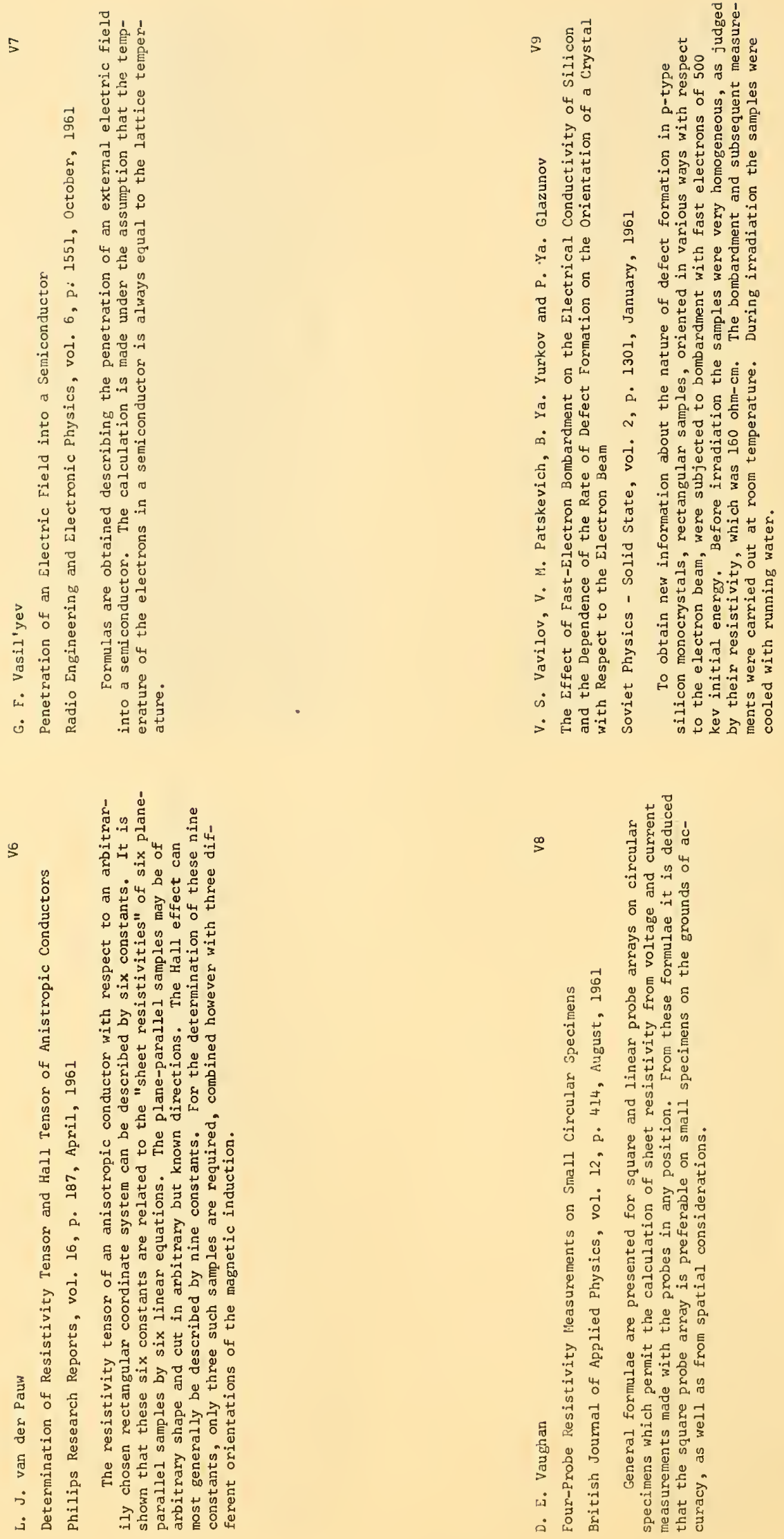

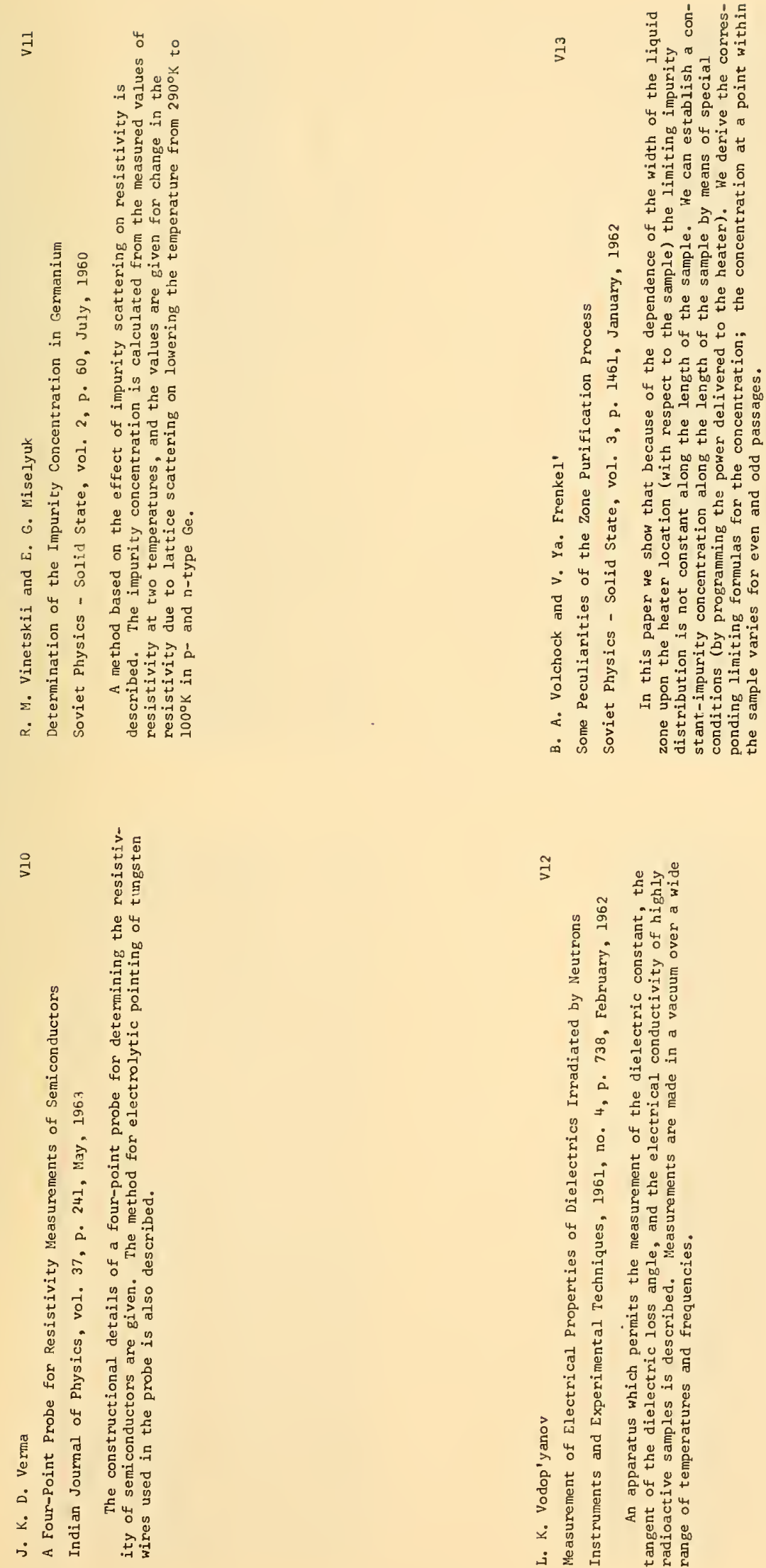

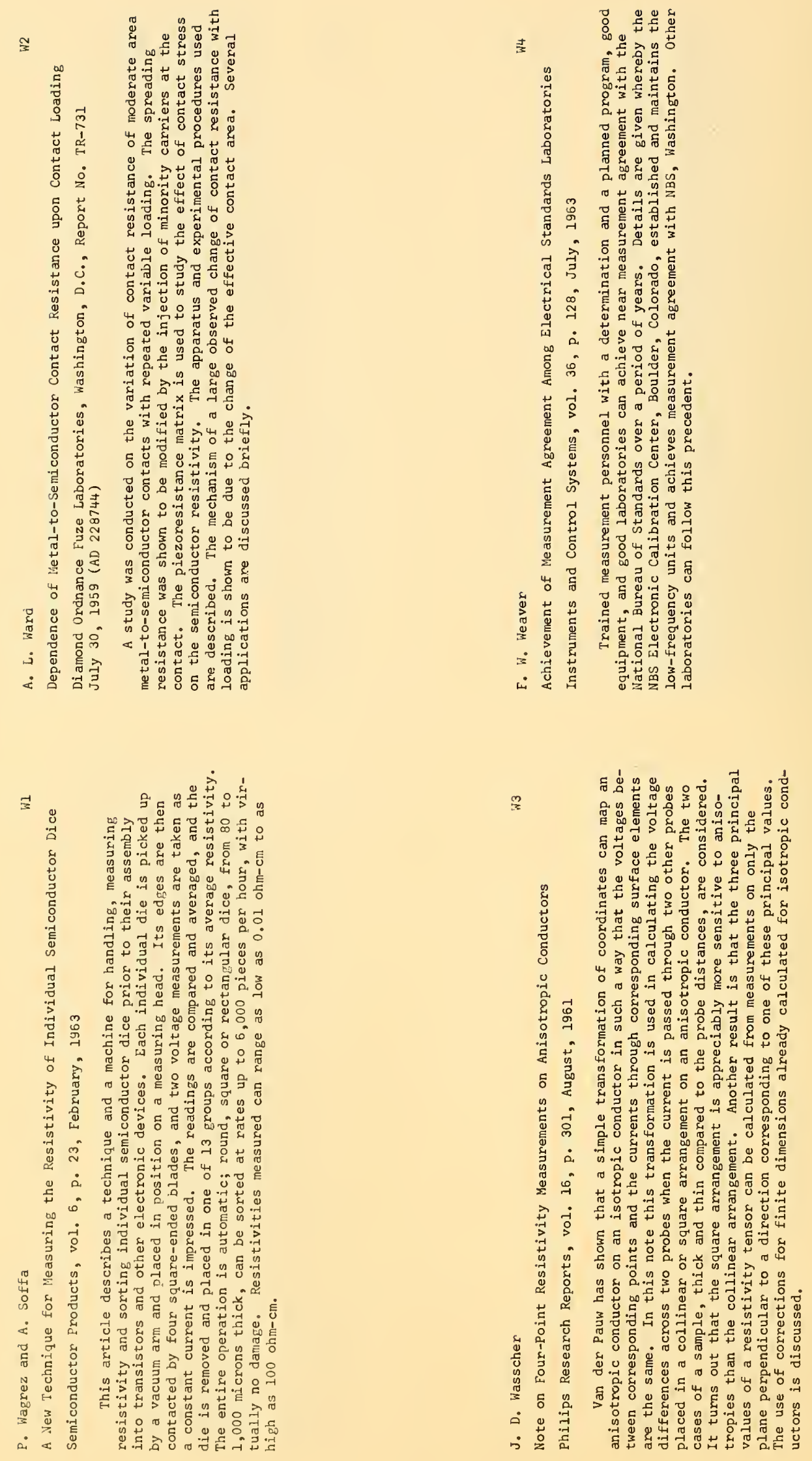


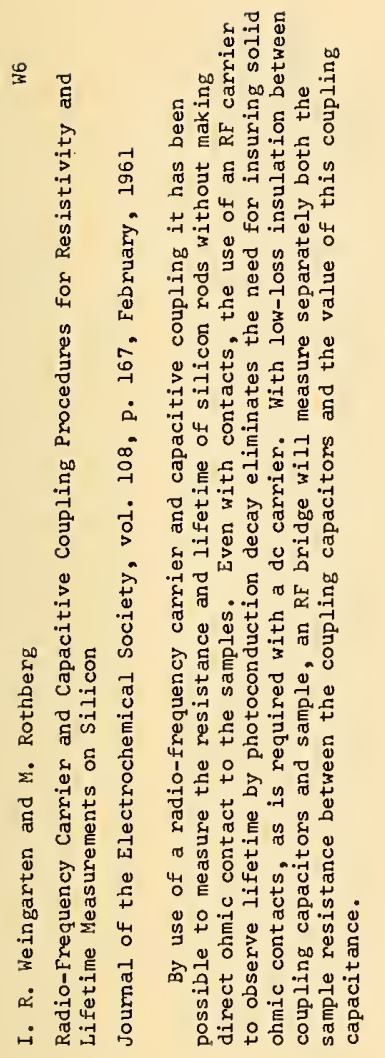

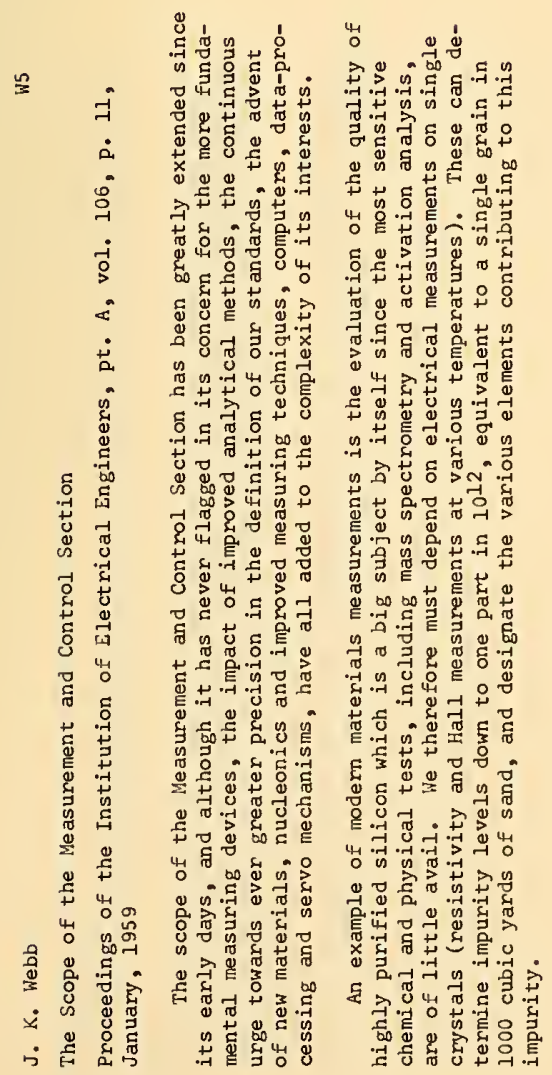
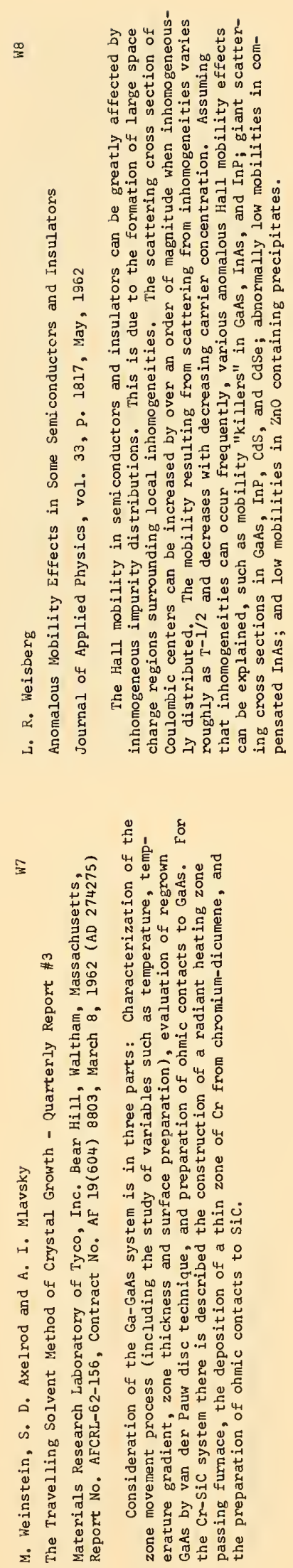

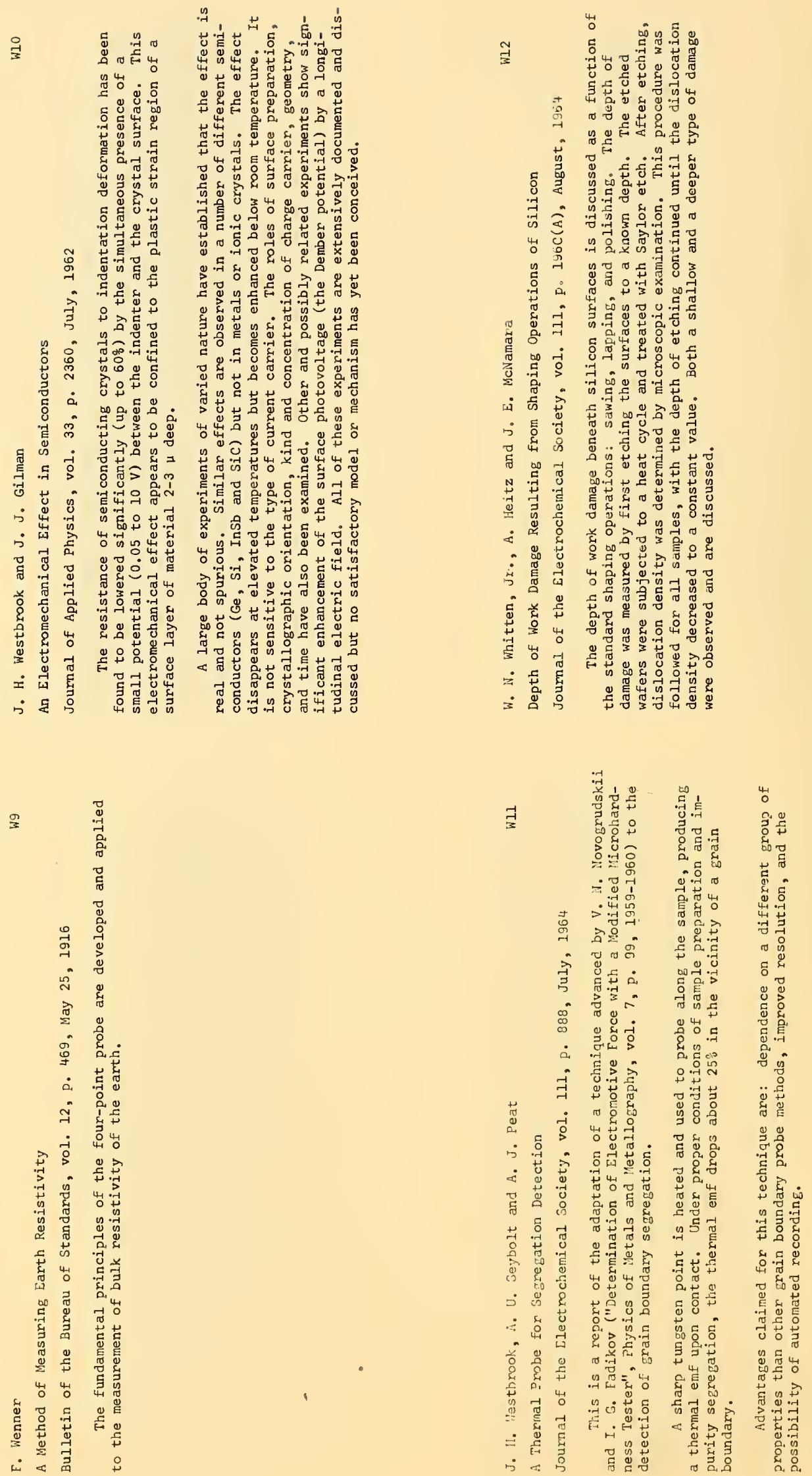

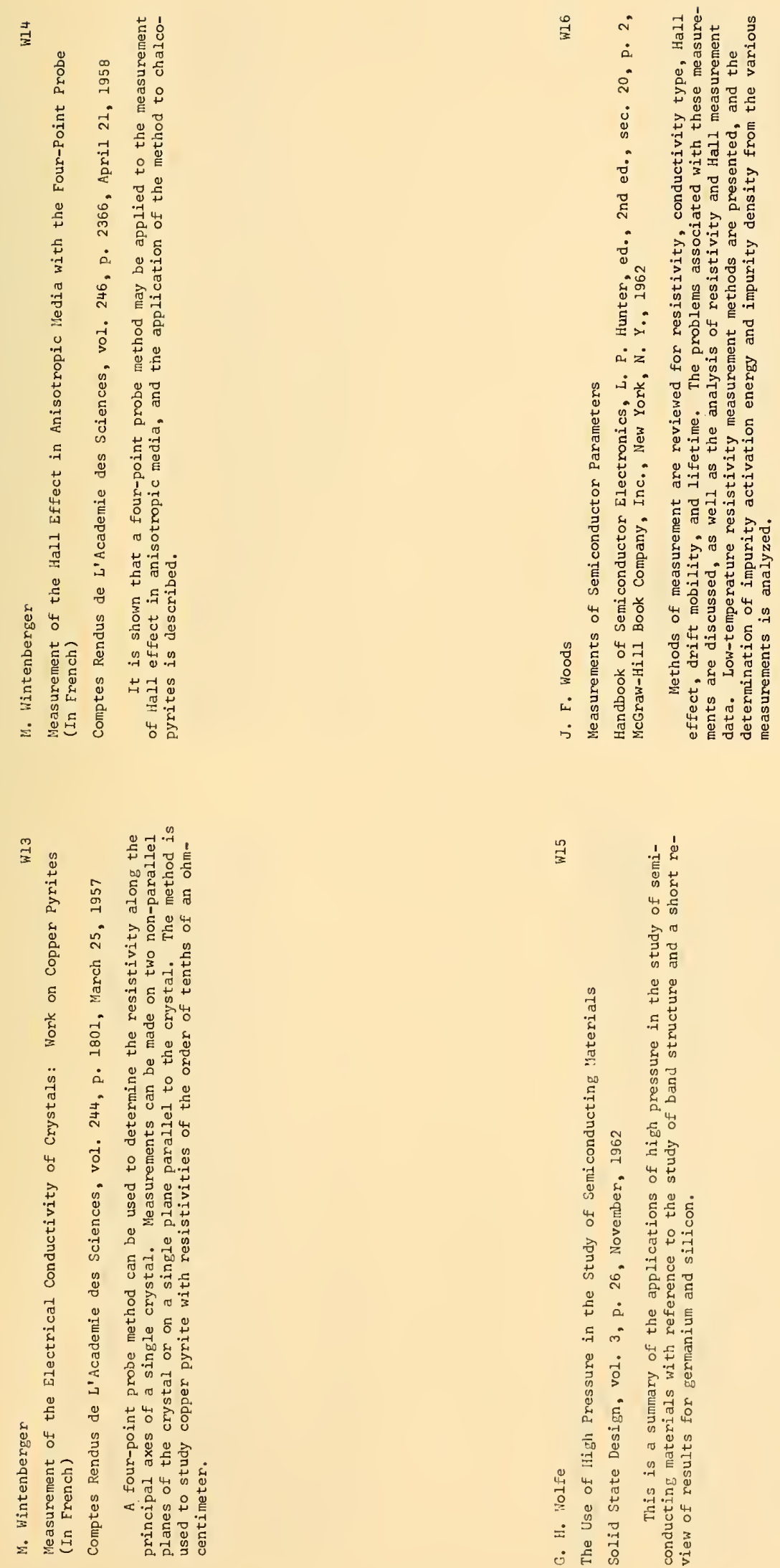

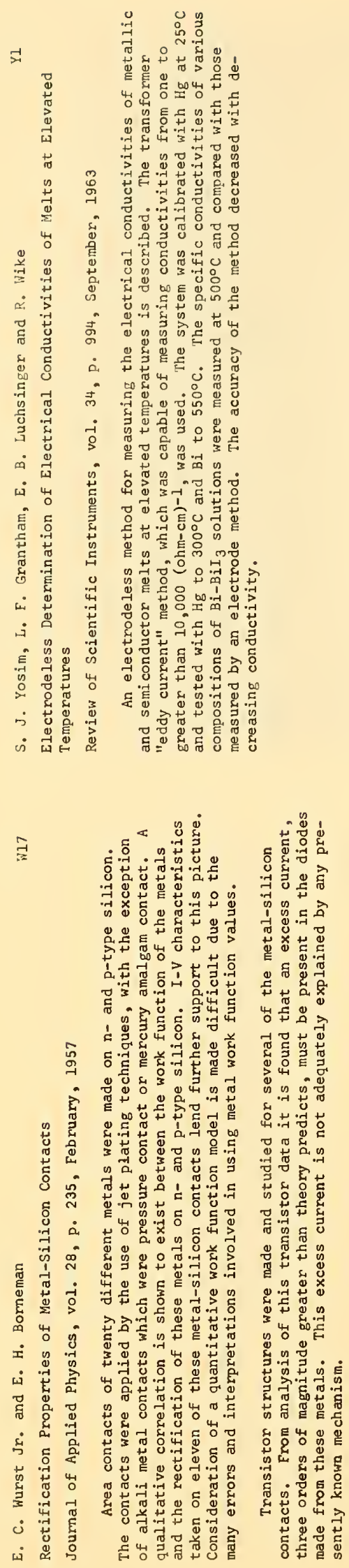
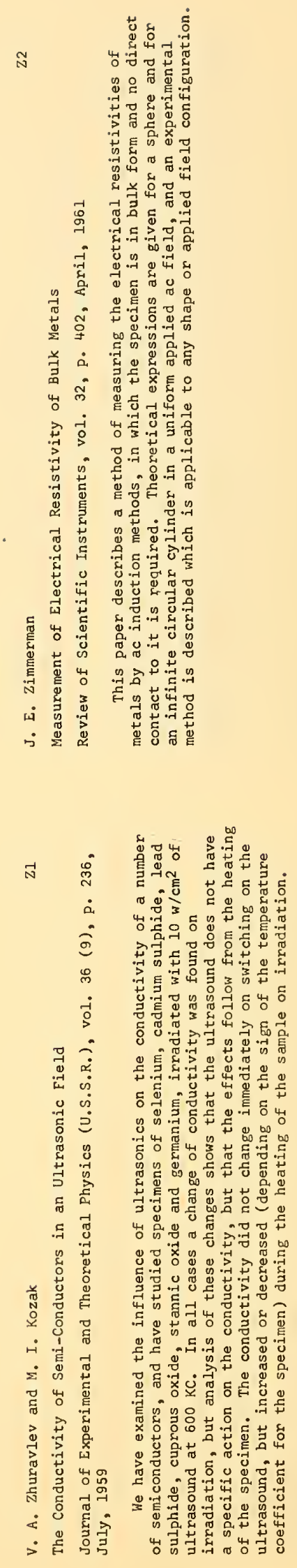


\section{ADDENDUM \\ to accompany}

NBS TECHNICAL NOTE 232

Bibliography on the Measurement of Bulk Resistivity of Semiconductor Materials for Electron Devices

\section{Judson C. French}

The following references, not included in the Bibliography, should be noted. Reference (1) should be assigned the identification number K4.I, added to the Index to Subject Matter under categories 3 and 7 , and added to the Author Index following Keller, J.B. Reference (2) should be assigned the identification number D16.1, added to the Index to Subject Matter under category 2, and added to the Author Index following Dunsmuir, R.

Reference 1:

W. Keller

Measurement of the Specific Resistance of Semiconductor Crystals Using High Frequencies (In German)

Zeitschrift flur angewandte Physik, vol. 11, P. 346, September, 1959

Several methods for the measurement of resistivity of semiconductor materials using high frequency techniques are briefly reviewed. These include an induced current method, a two-point (currentless voltage probe) method, an oscillator method and a bridge method. A method is then described in detail for measurements of rod-shaped samples in which no contact is made to the silicon crystal. The crystal may be wrapped in a protective material. Resistance is indicated by the degree of damping of a tuned circuit which is capacitively coupled to the sample. It is necessary to calibrate the measurement using crystals of known resistivity.

Reference 2:

J. Dušek

Measurement of the Hall Coefficient and Electrical Conductivity in Semiconductors by the Method of an Altemating Magnetic Field and Alternating Current

Czechoslovak Journal of Physics, vol. 9, P. 250, 1959

A description is given of electronic equipment which enables the simultaneous measurement of the Hall coefficient and the electrical conductivity by using a low frequency altemating magnetic field and alternating current. The instrument is adapted for measuring a minimum $\mathrm{Hall}$ coefficient of $3 \times 10^{-11} \mathrm{Vcm} / \mathrm{AG}$ and electrical resistance $10^{-5} \Omega$ to $10^{3} \Omega$; the smallest measurable Hall voltage is $1 \mu \mathrm{V}$. Deviations from the results obtained by the classical dc method do not exceed $5 \%$. 


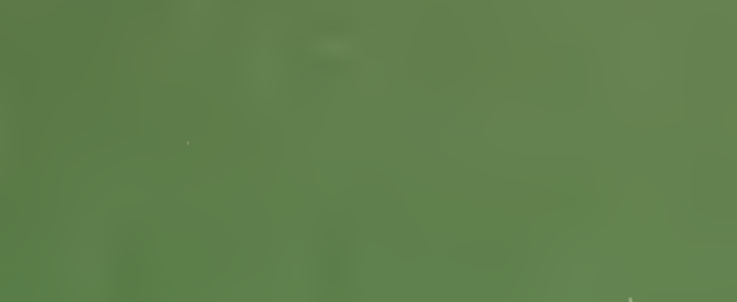


UNITED STATES

OVERNMENT PRINTING OFFICE

DIVISION OF PUBLIC DOCUMENTS

WASHINGTON, D. C., 20402
PENALTY FOR PRIVATE USE TO AVOID PAYMENT OF POSTAGE. $\$ 300$ (GPO)

OFFICIAL BUSINESS

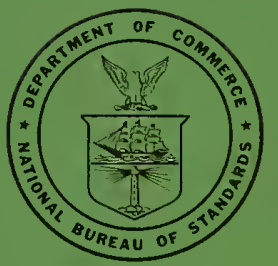

